Target Localization Using MIMO-monopulse

Application on 79 GHz FMCW Automotive Radar

J. Ruoyu Feng 



\section{TARGET LOCALIZATION USING MIMO-MONOPULSE \\ Application on 79 GHz FMCW Automotive Radar}

by

\section{J. Ruoyu Feng}

in partial fulfillment of the requirements for the degree of

\section{Master of Science}

in Electrical Engineering

at the Delft University of Technology,

to be defended publicly on Thursday November 30, 2017 at 10:00 AM.

$\begin{array}{lll}\text { Supervisor: } & \text { Dr. F. Usyal } & \\ \text { Thesis committee: } & \text { Prof. A. Yravovy, } & \text { TU Delft } \\ & \text { Dr. F. Usyal, } & \text { TU Delft } \\ & \text { Dr. R. Huesdens, } & \text { TU Delft }\end{array}$

This thesis is confidential and cannot be made public until November 30, 2018.

An electronic version of this thesis is available at http://repository . tudelft.nl/. 



\section{Abstract}

Automotive radar is widely used for driving safety support and it is a key element of future autonomous vehicles. Radar sensors have the property that the performance is not to be affected by low vision conditions compared with camera sensors or laser based radar sensors, which makes it crucial for the autonomous driving system.

Automotive radar system utilizes millimeter-wave band to detect the range, velocity and direction of arrival (DOA). Commercial $24 \mathrm{GHz}$ and $77 \mathrm{GHz}$ radar have been well developed in vehicle applications at present, and the future trend is $79 \mathrm{GHz}$ solution with wider $4 \mathrm{GHz}$ bandwidth, which has been defined by the European Commission in 2004 as the frequency allocation for automotive shortrange radar systems. The main modulation method of automotive radar is Frequency-Modulated Continous-Wave(FMCW), which holds advantages including high-resolution distance measurement, quick updating and lower sampling frequency required on hardware.

The resolution and accuracy of DOA estimation depend on the number of used antennas and their physical size. To improve the performance advanced algorithms and antenna configurations can be used. Socalled super-resolution techniques can improve the estimation performance but suffer when few measurements are available and the number of targets is unknown, and the performance will also be degraded by correlated signals.

Monopulse is an established technique for radars for precision angle estimation. It enables DOA realized in only one pulse and requires quite less computation complexity. Conventional monopulse with one transmitter and two receivers and phased array monopulse have been well developed especially in tracking radar system. MIMO-monopulse has been studied in some literature, but most of them utilize distributed MIMO. The research and application of colocated MIMO-monopulse are still in progress.

In FMCW automotive radar application, clutter may have a strong effect on DOA detection and estimation of targets with low RCS, e.g. pedestrian. With Space-Time Adaptive Processing(STAP), it is possible to suppress the clutter in the angle-Doppler domain. MIMO-monopulse basically utilizes digital beamformer to generate sum and difference channel, which also allows STAP processing to obtain adaptive sum and difference weighting. With STAP clutter can be canceled, thus false detection of MIMO-monopulse will be avoided.

In this master thesis, we propose the solution of angle estimation algorithm using MIMO-monopulse based on an actual automotive radar provided by NXP using colocated MIMO antenna. The algorithm is simulated through Matlab and verified on real experimental data. The performance of the algorithm is compared with conventional DOA algorithm and the advantages and disadvantages are analyzed. A feasible extension to STAP will be discussed to suppress the clutter for preventing false DOA estimation. Since monopulse estimator has been approved high performance in the field of single target tracking (such as low RCS pedestrian), 
our proposed algorithm is also validated in single and multiple target DOA estimations, which is an attractive scenario for a potential application using MIMO-monopulse. 


\section{ACKNOWLEDGEMENT}

Here I would like express my thanks of graduate to the people who help and support me while I am doing the master thesis.

Firstly, I would like to thank Prof. Alexandar Yarovoy, who formulated the topic of thesis for me. Thanks to his acadamic insight, I have an opportunity to make my master thesis a novelty research.

Secondly, I would like to thank my daily supervisor dr. Faruk Usyal. During my study of thesis, we had much discussion and he gives me valuable suggestions and guidance. When I get some trouble or report the progress, he always gives me feedback as soon as possible. The support is not only limited to the knowledge, but also a lot of experience and skill about scientific research.

I would like to also thank the technicians of the MS3 group for supporting me with the implementation of the algorithm and experimental validation. Without their assistance, the theory of the master thesis will never be realized on actual radar and verified using real data. Besides, I appreciated all the Phd students and other professors in our group for helpful advice.

Last but not least, I would like to thank my family for supporting me to study, and my friends for spending the wonderful time together in TU delft. 



\section{ConTEnTS}

List of Figures
ix

1 Introduction $\quad 1$

1.1 Motivation .............................. 2

1.2 Research Goal and Approach . . . . . . . . . . . . . . . . . . . . . 2

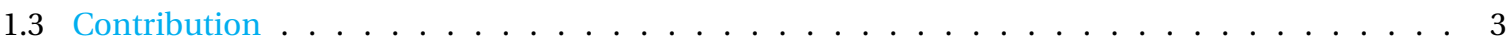

1.4 Organization of the thesis . . . . . . . . . . . . . . . . . . . 3

2 Liturature Study and Problem Formulation $\quad 5$

2.1 FMCW radar and Signal processing . . . . . . . . . . . . . . . . . . . . 5

2.1.1 Sawtooth Modulation Method . . . . . . . . . . . . . . . . . . . . . 6

2.1.2 Triangular Modulaion Method . . . . . . . . . . . . . . . . . . . . 8

2.2 Multiple-input Multiple-output Radar . . . . . . . . . . . . . . . . . . . . . . . 9

2.2 .1 Orthogonal Waveforms Generation ． . . . . . . . . . . . . . . . . . . . 10

2.2.2 MIMO Array Model . . . . . . . . . . . . . . . . . . . . 12

2.2.3 Review of MIMO DOA Estimation Algorithm. . . . . . . . . . . . . . . . . . . 13

2.3 Monopulse Fundamentals . . . . . . . . . . . . . . . . . . . . . . . . . 14

3 DOA estimation using MIMO-monopulse $\quad 17$

3.1 Digital Beamforming $(\mathrm{DBF}) \ldots \ldots \ldots \ldots \ldots \ldots$

3.1.1 Narrow Band Beamforming . . . . . . . . . . . . . . . . . . 17

3.1 .2 The Effect of Wideband . . . . . . . . . . . . . . . . . . . . . . . 19

3.2 MiMo Monopulse . . . . . . . . . . . . . . . . . . . . . . . . 22

3.2.1 Amplitude Comparison . . . . . . . . . . . . . . . . . . . . 22

3.2.2 Phase Comparison . . . . . . . . . . . . . . . . . . . 25

3.3 Synthesis of Sum and Difference Pattern . . . . . . . . . . . . . . . . . . . . 27

3.3.1 Dolph-Chebyshev Sum pattern . . . . . . . . . . . . . . . . . . . . . . 28

3.3.2 Zolotarev Difference Pattern . . . . . . . . . . . . . . . . . . . . . . 29

4 Clutter Suppression Using STAP Adaptive MIMO-monopulse 3

4.1 Space-time Signals . . . . . . . . . . . . . . . . . . . . . . . . . 34

4.2 Space-time Adaptive Monopulse . . . . . . . . . . . . . . . . . . . . . . . . 36

4.2.1 Adaptive Digital Beamformer . . . . . . . . . . . . . . . . . . . 36 
4.2.2 Space-time Adaptive Sum and Difference Beamformer . . . . . . . . . . . . . . . 38

5 Implementation and Performance Evaluation $\quad 4 \mathbf{4 1}$

5.1 Implementation Based on Matlab Simulation. . . . . . . . . . . . . . . . . . . . . 41

5.1 .1 FMCW Signal Generation . . . . . . . . . . . . . . . . . . . . . 41

5.1.2 Processing Using 3D Fast Fourier Transform ． . . . . . . . . . . . . . . . . . . 42

5.1 .3 Digital Beamforming. . . . . . . . . . . . . . . . . . . . 44

5.1 .4 MIMO-monopulse. . . . . . . . . . . . . . . . . . . . 44

5.1.5 Comparison with Super-resolution Algorithm ． . . . . . . . . . . . . . . . . . . . 48

5.1.6 Performance Evaluation . . . . . . . . . . . . . . . . . . . . 49

5.1 .7 STAP MIMO-Monopulse . . . . . . . . . . . . . . . . . . . . . . 51

5.2 Experimental Data Processing . . . . . . . . . . . . . . . . . . . . . 52

5.2 .1 Calibration of MiMO Array. . . . . . . . . . . . . . . . . . . . 53

5.2.2 Moving Target DOA Estimation using MIMO-monopulse . . . . . . . . . . . . . . . 59

6 Conclusion $\quad 63$

6.1 Future Work. . . . . . . . . . . . . . . . . . . . . . . . 64

$\begin{array}{lr}\text { Bibliography } & 67\end{array}$ 


\section{LIST OF FIGURES}

2.1 Frequency changing with respect time: transmitted and received chirp . . . . . . . . . . 7

2.2 Fast time and slow time of 2 D radar data matrix $\ldots \ldots \ldots \ldots \ldots \ldots$

2.3 Real chirp signal frequency changing with time $\ldots \ldots \ldots \ldots \ldots \ldots$

2.4 Comparison between MIMO with phased array. MIMO radar transmits independent signals,

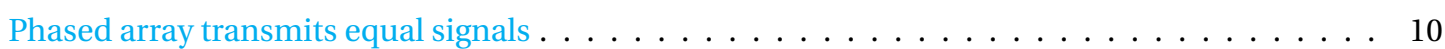

2.5 Time Division Multiplexing, each channel transmits chirp sequentially . . . . . . . . . . . 11

2.6 Virtual array with 12 elements, the number of transmitter and receiver are $M_{t}=3, M_{r}=4$. The blue solid antennas represent the transmitter elements, and the blue dashed antennas represent the virtual elements generated from red receiver antennas . . . . . . . . . . . . . . . 12

2.7 3D data cube of FMCW MiMO signal model . . . . . . . . . . . . . . . . . . . . . 13

2.8 Monopulse classification: amplitude and phase comparison . . . . . . . . . . . . . 15

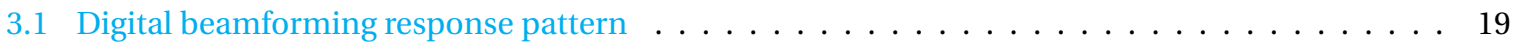

3.2 Wideband DBF response pattern, beam width decreases with frequency increasing . . . . . . 20

3.3 Wideband beamforming, the beam is steered to $-60^{\circ} \ldots \ldots \ldots \ldots \ldots \ldots \ldots \ldots$

3.4 Left beam and right beam of amplitude comparison monopulse . . . . . . . . . . . . . 23

3.5 Sum and difference beam of amplitude comparison monopulse . . . . . . . . . . . . . . 24

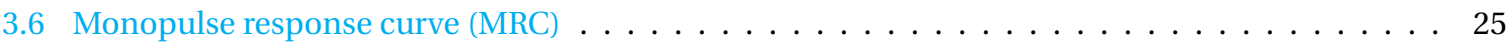

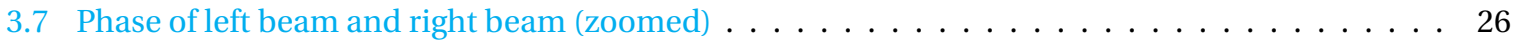

3.8 Sum and difference pattern, monopulse ratio using phase comparison method . . . . . . . . 27

3.9 Dolph-Chebyshev sythesis of sum pattern of 12 elements, sidelobe level is $-40 \mathrm{~dB}$. . . . . . . 29

3.10 Approximation to Zolotarev sythesis of sum pattern of 12 elements, $a=0.65 \ldots \ldots$. . . . . . 31

3.11 Sum and difference pattern, monopulse ratio using Chebyshev and Zolotarev synthesis . . . . 32

4.1 Space time signals in 3D radar data cube, which is obtained by extracting spatial and slow-time snapshots in one range cell and reshape the matrix into vector . . . . . . . . . . . . . 35

4.2 Adaptive beamforming. A jammer is from $-25^{\circ} \ldots \ldots \ldots \ldots \ldots \ldots$

5.1 Simulation of processing FMCW signals using 3d FFT. Three targets with different range,velocity and DOA are visualized in range-Doppler and range-azimuth map . . . . . . . . . . . . . . 43

5.2 Range-azimuth map of DBF . . . . . . . . . . . . . . . . . . . . . . . . . . 44

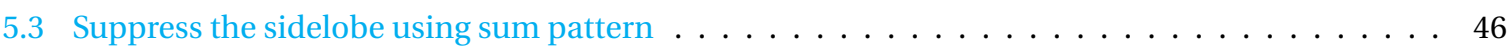


5.4 Range azimuth after the proposed calibration, and test on different data sets . . . . . . . . . . 47

5.5 Single snapshot effect, SNR is $15 \mathrm{~dB} \ldots \ldots \ldots \ldots \ldots \ldots \ldots \ldots \ldots \ldots$

5.6 Wrong number of targets estimated $\ldots \ldots \ldots \ldots \ldots \ldots \ldots \ldots \ldots$

5.7 Root mean square error of 3 targets in the same range cell . . . . . . . . . . . . . . . . 50

5.8 Root mean square error of tracking the target using MRC with fixed beam, SNR is $15 \mathrm{~dB}$ and the

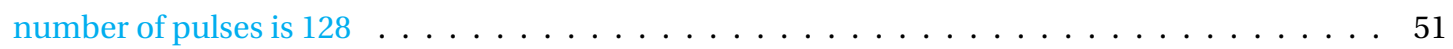

5.9 Angle-Doppler map of STAP MIMO-monopulse, sum beam . . . . . . . . . . . . . . . . 52

5.10 Angle-Doppler map of STAP MIMO-monopulse, difference beam . . . . . . . . . . . . . . 52

5.11 Transmitter(upper 3 elements) and Receiver(lower4 elements) array of Dolphin . . . . . . . . . 53

5.12 Range azimuth map, target at $6.8 m$ and $0^{\circ} \ldots \ldots \ldots \ldots \ldots \ldots \ldots$

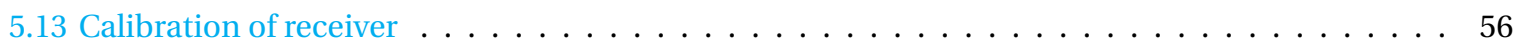

5.14 Range azimuth map, target at $6.8 \mathrm{~m}$ and $0^{\circ}$. (a) is the measurement at $0^{\circ}$ and (b) is after calibration using $\mathrm{NXP}$ samples. . . . . . . . . . . . . . . . . . . . . . . . . 57

5.15 Range azimuth (DBF) after the proposed calibration, and test on different data sets . . . . . . . 59

5.16 Range azimuth maps: Comparison using MIMO-monopulse and traditional method . . . . . . 61 


\section{1}

\section{INTRODUCTION}

In Europe alone, about 1.3 million traffic accidents cause more than 41,000 fatalities and an economic damage of more than 200 billion Euros per year. Human error is involved in over $90 \%$ of the overall accidents [1]. The concept of autonomous driving is expected to dramatically reduce the traffic collision and provide much increased safety. It is currently the hottest technology trend and it is coming soon to be realized.

Automotive radar is the key element for a driveless car, which has been recently developed and widely used in advanced driver assistance systems. A number of different technologies have been used in active safety systems to monitor the surrounding environment of a vehicle such as collision avoidance, blind spot detection, and parking aid. Compared with other sensors in self-driving systems like camera and LIDAR, Millimeter wave radar has advantages of robust performance against low vision environmental situations such rough weather and limited lighting conditions. Automotive radar is crucial for the autonomous car so that the market of automotive radar is expected to keep largely increasing in the future.

Radar is an object-detection system that uses radio waves to determine the range, and velocity of objects. The abbreviation radar stands for "radio detection and ranging". The radar utilizes the electromagnetic waves to extract the information of the targets. Basically, the range can be determined through the measurement of delay caused by reflection from targets, and velocity is calculated according to the property of Doppler effect. Depending on the antenna configuration, it is even possible to accurately detect the angle of targets using more than one receive or transmit channel.

The main modulation method of automotive radar is Frequency-Modulated Continous-Wave (FMCW), which holds advantages including high resolution distance measurement, quick updating and low sampling frequency requirements of analog-to-digital converter. An FMCW radar system generates frequency modulated signals which enable to measure the distance with simple processing in hardware.

In automotive radar application, MIMO radar technology is used to obtain the angular information of 
targets. Direction of arrival(DOA) estimation is a significant issue in automotive radar signal processing. In order to realize it through real-time processing for quick response autonomous driving system, quick and accurate estimation of DOA is a basic requirement of such algorithm.

\subsection{MOTIVATION}

Automotive radar system utilizes millimeter-wave band to detect the range, Doppler velocity and direction of arrival (DOA). Commercial $24 \mathrm{GHz}$ and $77 \mathrm{GHz}$ automotive radar have been well developed and $79 \mathrm{GHz}$ is the future solution for short range radar which has been defined by European Commission in 2004. It has $4 \mathrm{GHz}$ bandwidth, thus will provide better range resolution. The DOA estimation algorithm to be used for $79 \mathrm{GHz}$, therefore, becomes a potential field of research.

The angular resolution and accuracy of estimation depend on the physical size and the number of antenna elements used in a sensor array. So-called super-resolution algorithms such as MUSIC and ESPRIT can improve the estimation performance but suffer when few measurements are available, since the subspacebased algorithms require an estimation of noise covariance. Moreover, such eigenstructure analysis requires to the known number of targets to exactly determine the effective rank of the correlation matrix, which is not realistic in practical application. Last but not least, the computational cost of eigenvalue decomposition is quite large, which is not desired in a real-time processing and quick response automotive radar system.

Monopulse is a well established technique for radars on precision angle estimation. Compared with a subspace-based algorithm, monopulse has potential benefits of saving computation cost, no requirements to know the number of targets and capability to work in limited snapshots, meanwhile better accuracy than conventional digital beamforming. Moreover, it naturally provides convenience in target tracking, which is also attractive in the automotive application such as pedestrian tracking.

Conventional monopulse with one transmitter and two receivers and phased array monopulse has been well developed especially in tracking radar system. The concept of MIMO-monopulse has been studied in some literature, but most of them utilize distributed MIMO with widely separated transmitters and receivers. The research and application of colocated MIMO-monopulse such as in automotive radar are not commonly considered and still potential to investigate.

\subsection{ResEARCH GOAL AND APPROACH}

The research goal of the master thesis is to propose a direction of arrival(DOA) estimation algorithm by utilizing MIMO-monopulse technique on the $79 \mathrm{GHz}$ FMCW automotive radar for the target localization. To reach this goal, we realized the following approaches.

The simulation model of FMCW automotive radar signals is built, including chirp signal, transmitting and receiving delayed signals, white noise, and clutter. Then, deramp processing is applied on generated signals. Furthermore, multiple input multiple output array model is constructed to provide the direction of arrival information. Several targets with the different range, velocity and angle can be generated in this model, and the output is a $3 \mathrm{D}$ data cube including fast time, slow time and sensor domain. 
Preliminary processing of FMCW signals is adopted using 3D Fast Fourier Transform. Digital beamforming is applied on sensor domain to obtain better performance of DOA estimation, which is fundamental for MIMO-monopulse. The emphasis is on the derivation of MIMO-monopulse, and different methods of generating sum and difference beam will be compared. A methodology of synthesis of sum and difference beam is introduced, which is the proposed manner in this thesis.

As a feasible extension of MIMO-monopulse DOA estimation algorithm, STAP is considered to be combined with MIMO-monopulse to realize clutter and jammer suppression.

The signal model and processing algorithm are first simulated through MATLAB code, and then testified on actual radar measurement. The real data was collected by NXP Dolphin automotive radar test chip. To realize DOA estimation, calibration of MIMO antenna array was first implemented by using an indoor measurement. Since monopulse estimator has good performance on single target tracking, we also attempt to apply this algorithm on pedestrian tracking, which is attractive for safety autonomous driving. The algorithm is also validated on the real data processing collected on the car in an outdoor measurement.

\subsection{Contribution}

The main novelty of this thesis is to extend monopulse technique on MIMO radar for target localization application, which is not commonly considered by previous work, especially on FMCW automotive radar application. Furthermore, We propose to synthesize sum and difference pattern by using Chebyshev and Zolotarev windows to suppress sidelobe and improve the accuracy of estimation. We demonstrate the success of proposed method on real automotive radar data.

Besides, the MIMO-monopulse concept is combined with space-time adaptive processing (STAP) for clutter suppression. In this thesis, we investigate STAP MIMO-monopulse to mitigate ground clutter and multipath clutter which is a common scenario in the urban environment of driving.

\subsection{ORganization OF THE THESIS}

The structure of the thesis work is given as follows:

Chapter 2 is the literature study and problem formulation. The concept of automotive radar signal processing, Multiple input multiple output radar and Direction of arrival estimation problems are given. The state-of-the-art approaches of DOA estimation are briefly described. At last, we introduce monopulse technique for DOA estimation and explain the fundamental concept.

In Chapter 3, details of realizing MIMO-monopulse algorithm are discussed. To start with, digital beamforming is derived as a basic of MIMO-monopulse working on MIMO antenna array. Common methodologies of generating monopulse beam patterns are derived, and the synthesis of sum and difference pattern is proposed to improve the performance of MIMO-monopulse.

Chapter 4 extends the MIMO-monopulse to space-time adaptive processing for clutter suppression which generally exists in the automotive radar application.

Simulation and experimental analysis are given in Chapter 5. The FMCW MIMO signal model is built, 
and preliminary processing is applied on a data cube to obtain range, Doppler, and angle. MIMO-monopulse is used to process FMCW signals and compared with digital beamforming. For real radar data processing, experiment on ideal target in high SINR scenarios is designed to realized the calibration of MIMO array. Tracking of moving corner reflector is testified to demonstrate the performance of MIMO-monopulse on the potential application of tracking.

Finally, conclusion and future works are discussed in Chapter 6. Since MIMO-monopulse is a new concept for automotive radar application, there is much potential to further study and improve this algorithm. 


\section{Liturature Study and Problem}

\section{FORMULATION}

In this section, we give a description of the problem to be solved. To start with, the fundamentals of FMCW radar and signal processing is given. Then the multiple-input multiple-output(MIMO) radar is introduced. Further, the concept of DOA estimation will be explained, also some state-of-the-art algorithms will be reviewed. A brief introduction of monopulse technique is particularly discussed. Then we will extend these techniques for MIMO model in the following chapters.

\subsection{FMCW RADAR AND SIGNAL PROCESSING}

Frequency modulated continuous wave radar has been widely used for automotive radar systems including the safety applications such as adaptive cruise control, collision avoidance, parking aid, crash mitigation, stop-and-go, and pre-crash sensing [2]. The advantages of FMCW radars in comparison to pulse radars are the low measurement time and low peak-to-average power ratio [3].

The basic concept of automotive FMCW radars to generate a frequency modulated ramp as transmit signal, which in the automotive application is always linear. The primary processing of FMCW signals is called stretch processing (or deramp, dechirp), which means the received signal is transformed into sinusoidal one through a mixer. The difference frequency, or beat frequency, between the transmitted and received signal is determined to obtain the range and velocity information, which is composed of propagation delay and Doppler frequency [4].

Two different frequency bands are predominantly used for automotive radar: the $24 \mathrm{GHz}$ and $77 \mathrm{GHz}$ bands [2]. $77 \mathrm{GHz}$ is used for long-range radar (LRR) applications and $24 \mathrm{GHz}$ band radar which is used for a middle and short range. The $79 \mathrm{GHz}$ band from 77 to $81 \mathrm{GHz}$ will replace ultra wideband automotive radar 
sensors in the $24 \mathrm{GHz}$ band, as the most suitable frequency band for future short- and mid-range radar (SRR, MRR) automotive systems [5]. The conventional $77 \mathrm{GHz}$ automotive radar usually has $0.5 \mathrm{GHz}$ bandwidth, which cannot provide enough resolution in short range. Thus $79 \mathrm{GHz}$ radar utilizes a wideband of $4 \mathrm{GHz}$, which allows for high spatial resolution and a much better capability of distinguishing between objects. The FMCW radar generates a signal with a linear frequency change with respect to time. In general, there are two types methodology for FMCW modulation: Sawtooth and triangular.

\subsubsection{SAWTOOTH Modulation Method}

Sawtooth method uses only up-chirp frequency ramp in each repetition interval [4]. The transmit frequency for one ramp with bandwidth $B$ and duration $T$ where $-\frac{T}{2} \leq t \leq \frac{T}{2}$ can be expressed as:

$$
f=\frac{B}{T} t
$$

After integration from frequency into phase, the transmitted signal in intermediate frequency (IF) can be expressed in complex form as:

$$
\begin{aligned}
& \Phi(t)=\int_{\frac{T}{2}}^{\frac{T}{2}} \frac{B}{T} t \mathrm{~d} t \\
& s_{T, I F}(t)=\exp \{j 2 \pi \Phi(t)\}=\exp \left\{j 2 \pi\left(\frac{k}{2} t^{2}\right)\right\} .
\end{aligned}
$$

Where $k=B / T$. Such form of transmitted signal is also called ramp or chirp signal. where $f_{c}$ is the center frequency. The transmitted signal in radio frequency $(\mathrm{RF})$ is given as:

$$
s_{T, R F}(t)=\exp \left\{j 2 \pi\left(\frac{k}{2} t^{2}+f_{c} t\right)\right\}
$$

The received signal is defined as a delayed replica of the transmitted signal.If we define delay between the transmitted and received signal as $\tau$, then the received signal:

$$
s_{R, R F}(t)=\exp \left\{j 2 \pi\left(\frac{k}{2}(t-\tau)^{2}+f_{c}(t-\tau)\right)\right\} .
$$

After demodulation, 2.4 becomes:

$$
s_{R, I F}(t)=\exp \left\{j 2 \pi\left(\frac{k}{2}(t-\tau)^{2}+f_{c}(-\tau)\right)\right\} .
$$




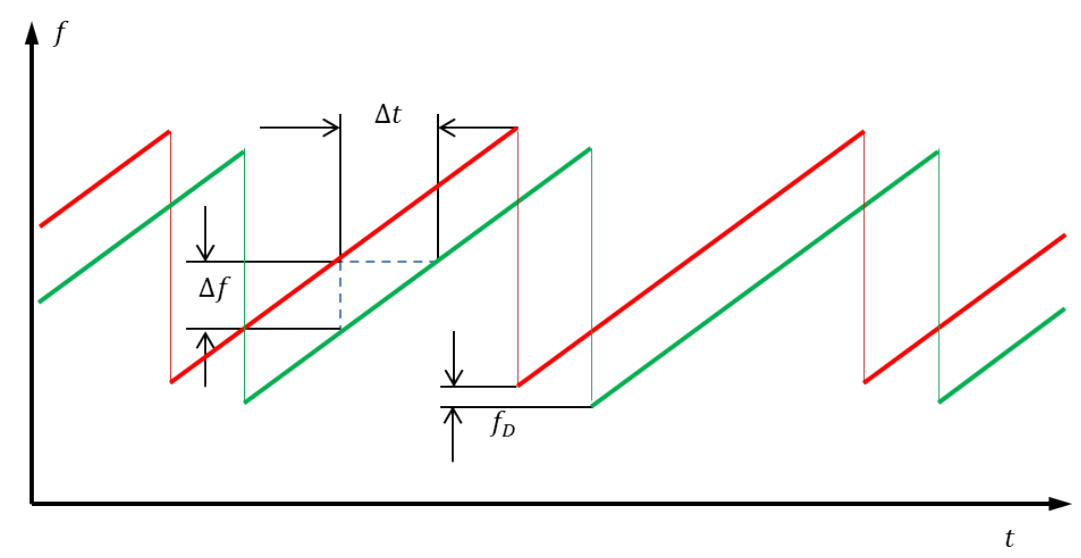

Figure 2.1: Frequency changing with respect time: transmitted and received chirp

The delay $\tau$ is defined by the round-trip delay of the waveform in free space as $\tau=2(R+v \cdot t) / c$, where $R$ and $v$ are the range and relative line-of-sight velocity of the target respectively, and the $c$ is the speed of light.

The processing of FMCW signal on radar-front end is called stretch processing, or known as deramp and dechirp. The received signal is deramped through multiplying the complex conjugate of it with transmitted signal (here the transmitted signal is called reference signal), and the output is called beat signal:

$$
\begin{aligned}
s_{\text {beat }}(t) & =s_{T}(t) \cdot s_{R}^{*}(t) \\
& =\exp \left\{j 2 \pi\left(k \tau t-\frac{k}{2} \tau^{2}+f_{c} \tau\right)\right\} .
\end{aligned}
$$

since $4(R+v \cdot t)^{2} \ll c^{2}, \tau^{2} \approx 0$. Then, it becomes

$$
s_{\text {beat }}(t)=\exp \left\{j 2 \pi\left(\frac{2 f_{c} \cdot R}{c}+\left(\frac{2 f_{c} \cdot v}{c}+\frac{2 k \cdot R}{c}\right) t+\frac{2 k v}{c} t^{2}\right)\right\} \text {, }
$$

where the last term of phase can also be neglected $\left(\frac{2 k v}{c} t^{2}\right) \approx 0$ ). Consequently the beat frequency generated becomes:

$$
f_{b}=\frac{2 f_{c} \cdot v}{c}+\frac{2 k \cdot R}{c}
$$

The beat frequency includes both terms of range $\left(\frac{2 k \cdot R}{c}\right)$ and velocity $\left(\frac{2 f_{c} \cdot v}{c}\right)$. Normally the velocity term in beat frequency is very small relative to the range term and can be neglected. The beat frequency is obtained through Fast Fourier transform on fast-time samples, and then range can also be calculated by the beat frequency. The transmitter repeatably generates FMCW signal pulses(a linear ramp is a pulse within the PRI), which allows for Doppler processing. To obtain the Doppler frequency $f_{D}$ of targets, the second FFT should be adopted on slow-time samples. Several pulses of received signals are stored in a data matrix, and range-Doppler map can be obtained through 2D FFT. The peak of 2D FFT spectrum will appear at $\left[f_{b}, f_{D}\right]=\left[\frac{2 k \cdot R}{c}, \frac{2 v \cdot f_{c}}{c}\right]$. 


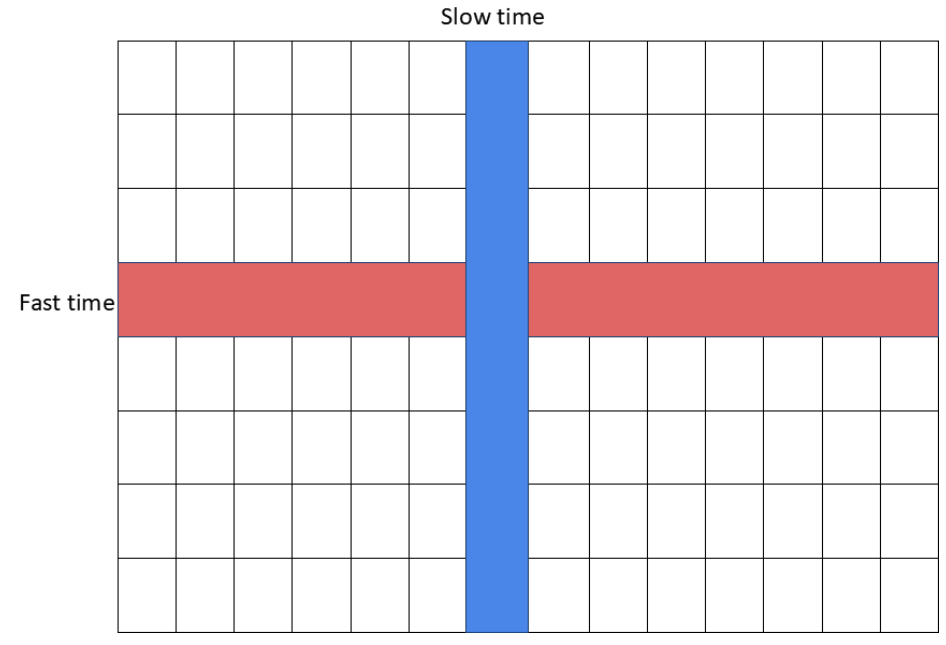

Figure 2.2: Fast time and slow time of 2D radar data matrix

In reality, the frequency of FMCW radar is not perfectly linear during sweep time. On our tested FMCW radar, the chirp will firstly start with a dwell period $t_{\mathrm{dwell}}$ during which the instantaneous frequency is equal to the initial frequency of the chirp $f_{\text {start }}$. The dwell time is followed by settle time $t_{\text {settle }}$ during which the phase-locked loop(PLL) converges to its linear operation mode. After this the data is sampled $t_{\text {sample }}$ in the data acquisition period which we actually used for signal processing(the instantaneous frequency reaches $\left.f_{\text {stop }}\right)$. After data acquisition, during the reset period $t_{\text {reset }}$, the PLL resets to a stable output frequency equal to the starting frequency. All these periods consists of a complete chirp period $T_{\text {chirp }}$.

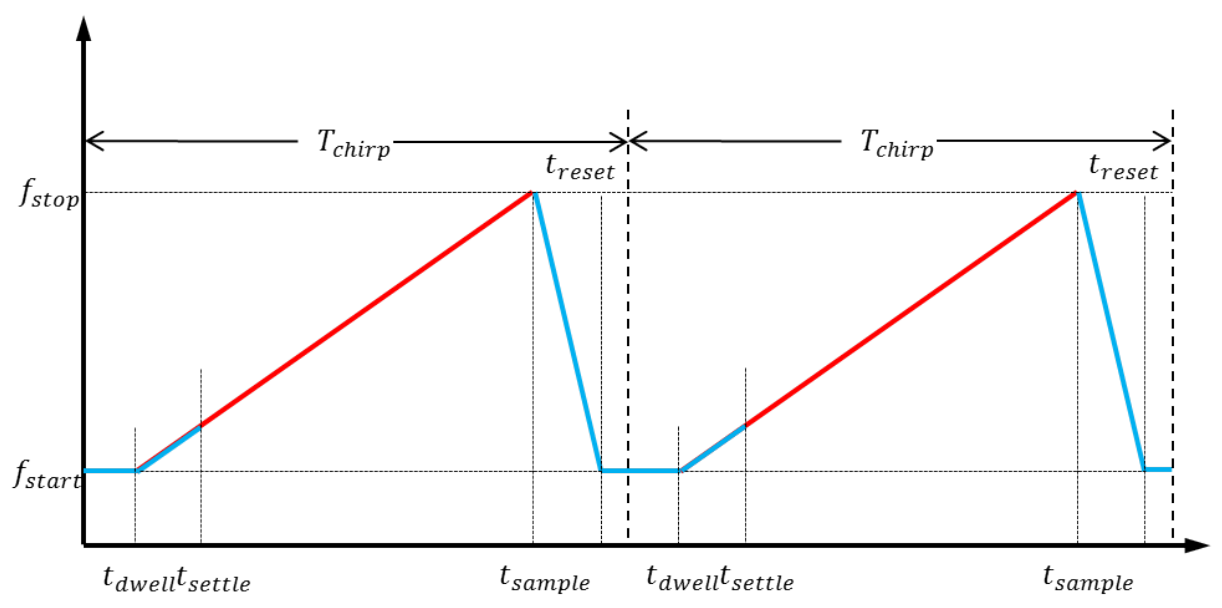

Figure 2.3: Real chirp signal frequency changing with time

\subsubsection{Triangular Modulaion Method}

Another popular FMCW modulation is called triangular method, which uses an up-chirp and down-chirp modulated frequency with in a ramp interval [6]. The advantage of triangular modulation is 2D FFT processing is not necessary. Consider a triangular modulation FMCW signal. Between [0,T/2], the up-chirp frequency is: $f_{u p}=\frac{B}{T} t$, and between $[T / 2, T]$, the down-chirp frequency is $: f_{\text {down }}=B-\frac{B}{T} t$. Similar to deriva- 
tion from (1) to (7), the beat frequency of up-chirp and down-chirp are respectively expressed as:

$$
\begin{aligned}
& f_{b+}\left(0<t<\frac{T}{2}\right)=\frac{2 f_{c} \cdot v}{c}+\frac{2 k \cdot R}{c} \\
& f_{b-}\left(\frac{T}{2}<t<T\right)=\frac{2 f_{c} \cdot v}{c}-\frac{2 k \cdot R}{c},
\end{aligned}
$$

From beat frequency, the range and velocity of the target can be calculated directly as:

$$
\begin{aligned}
& \nu=\frac{f_{b-}-f_{b+}}{2} \frac{c}{2 f_{c}} \\
& R=\frac{f_{b-}+f_{b+}}{2} \frac{c T}{2 B}
\end{aligned}
$$

Although 2D FFT is not required to reduce computational complexity, the main challenge of triangular modulation is multiple targets. Assuming that there exists $\mathrm{N}$ targets, thus there are $N$ beat frequencies in the up $\operatorname{ramp}\left(f_{b+1}, f_{b+2}, \ldots, f_{b+N}\right)$ and $\mathrm{N}$ beat frequencies in the down $\operatorname{ramp}\left(f_{b-1}, f_{b-2}, \ldots, f_{b-N}\right)$. Each up ramp beat frequency $\left(f_{b+i}, i=1,2, \ldots, N\right)$ and each down ramp beat frequency $\left(f_{b-j}, j=1,2, \ldots, N\right)$ can be a matched pair. Therefore, it gives rise to $N^{2}$ kinds of possible results $\left(R_{i j}, v_{i j}, \forall i, j=1,2, \ldots, N\right)$. However, only $N$ out of $N^{2}$ are real targets and the rest are so-called ghost targets. The strategy of using using ramps with different slopes and using Trapezoidal wave modulation are discussed in several literature to solve the ghost targets scenarios. [79] The problem of ghosts increases the complexity of triangular modulation FMCW radar, which will not be considered in this thesis.

\subsection{Multiple-input Multiple-output Radar}

Multiple-input multiple-output (MIMO) radar is an emerging technology that utilizes multiple elements in transmitter and receiver array. The MIMO radar systems can be classified into two categories: MIMO radar with colocated antennas, or coherent MIMO which provides an improved angular resolution of the array [10], and with widely separated antennas, or statiscal MIMO which has higher detection performance [11]. In FMCW MIMO radar systems, we utilize the concept of colocated MIMO radar in application of direction of arrival estimation.

Colocated MIMO radar is an extension to conventional phased array radar. The main difference compared to a standard phased-array radar is the capability of transmitting uncorrelated signals on multiple transmitter elements, while the phased array radar only transmits copies of a signal on each element. Such signals transmitted independently to each other on each transmitter element is called orthogonal waveforms, which allows the receiver array to separate these signals. Another big difference is that phased array radiates the electromagnetic wave to a specific direction through the control of phase shifter, while in MIMO array, each element is omnidirectional because the orthogonal waveforms emits energy in all directions with similar power [12]. .And the beam of the direction is shifted by digital signal processing in both transmitter and receiver. The difference between standard phased array radar and MIMO radar is illustrated in Figure 2.4.

In MIMO systems, the configuration of multiple elements in transmitter and receiver array creates virtual positions of elements and receiving channels through spatial convolution of transmitter and receiver phase centers [13], which is called virtual element and virtual array. This property increases the effective number 
of elements compared with the conventional radar using the same number of real antennas, thus further improves angular resolution. Or in another word, MIMO antenna array leads to larger virtual array aperture.

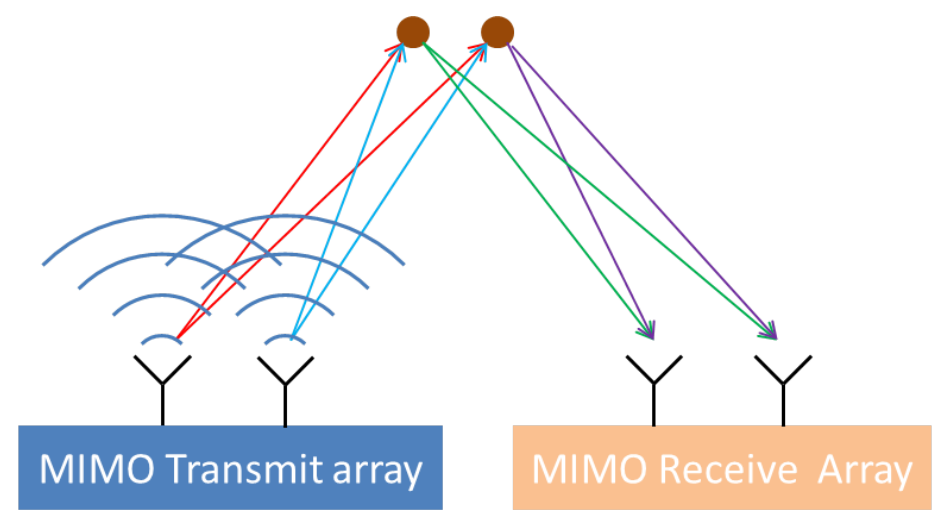

(a) MIMO

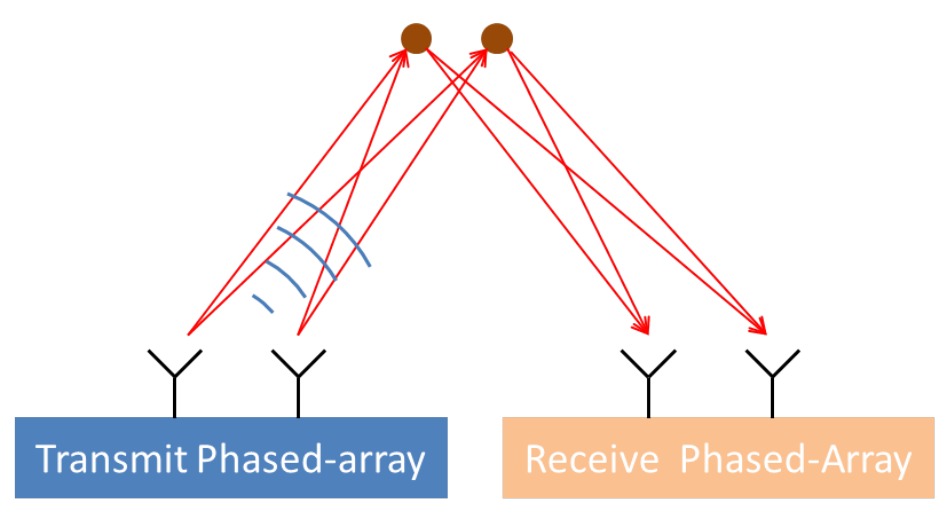

(b) Phased Array

Figure 2.4: Comparison between MIMO with phased array. MIMO radar transmits independent signals, Phased array transmits equal signals

\subsubsection{Orthogonal WAVEForms Generation}

In FMCW MIMO radar systems, orthogonal waveforms are generated with respect to each transmitter. Basically, there are 3 methods to generate orthogonal waveforms for MIMO radar system: time division, frequency division, and code division.

Time division is the most straightforward method to generate orthogonal waveforms. The transmitter antenna transmits sequentially. The receiver receives the signals which correspond to a single transmitter. For frequency division, the frequency bandwidth is divided for each transmitter which makes transmitter has no overlap in the spectral domain. All transmitters transmit simultaneously, which requires higher sampling rate. Code division generates orthogonal waveforms through codes with very low autocorrelation, which allows different transmitters share the same band of frequency and thus requires no increase in sampling rate.

In FMCW MIMO radar system, in [14] there are 3 methods of generating orthogonal waveforms: division 
by Beat-Frequency Offset, division by Chirp-Rate Offset, and Code division. In case of beat-frequency division, the transmitted waveforms are modulated with a frequency shift from each other. Given the transmitted signal, introduce a frequency shift $\Delta f$ and plus a phase term, which makes the transmitted signals orthogonal to each other. The expression of beat frequency-division signals is expressed as:

$$
s(t)=\exp \left\{j 2 \pi\left[\left(f_{c}-\Delta f_{b}\right) t+\frac{k}{2} t^{2}+\frac{\Delta f_{b}^{2}}{2 k}\right]\right\},
$$

$\Delta f_{b}$ is the frequency offset introduced for generating orthogonal waveforms. The last term in the exponential corresponds to residual video phase compensation, which is necessar for coherent receiver pocessing.

In case of chirp-rate division, the chirp rate of the transmitted waveform is modified with an offset, which is expressed as:

$$
s(t)=\exp \left\{j 2 \pi\left(f_{c} t+\frac{k+\Delta k}{2} t^{2}\right)\right\} .
$$

The chirp rate offset is given by $\Delta k$, which is obtained by changing the duration of the pulse. In order to maintain range resolution, the overall signal bandwidth must be the same for all transmitters. As a consequence, the chirp time must be different for each transmitter. in case of code division, The transmitted waveforms are coded with a binary phase shift keying (BPSK) signal with a low update rate:

$$
s(t)=\exp \left\{j 2 \pi\left(f_{c} t+\frac{k}{2} t^{2}\right)+0.5 \beta(t)\right\},
$$

where $\beta(t)$ is the BPSK signal with a low update rate that assumes values 1 and -1 .

Alternatively, another approach of generating nearly orthogonal waveforms is recommended in [15]. A set of continuous LFM signals having the same shape, but a relatively time-shifted (delayed from one another) one is proposed for use, which can be written as

$$
s(t)=\exp \left\{j 2 \pi\left(f_{c}\left(t-t_{s h}\right)+\frac{k}{2}\left(t-t_{s h}\right)^{2}\right)\right\},
$$

where $t_{s h}$ is the time offset between adjacent LFM signals.

In this thesis, time division method of generating orthogonal waveform is selected because of its simplicity of realization and perfect orthogonality. The time division orthogonal waveforms is realized that element of the transmitter array sequentially turns on and turn off to transmits signals, which is illustrated in Figure 2.5 .

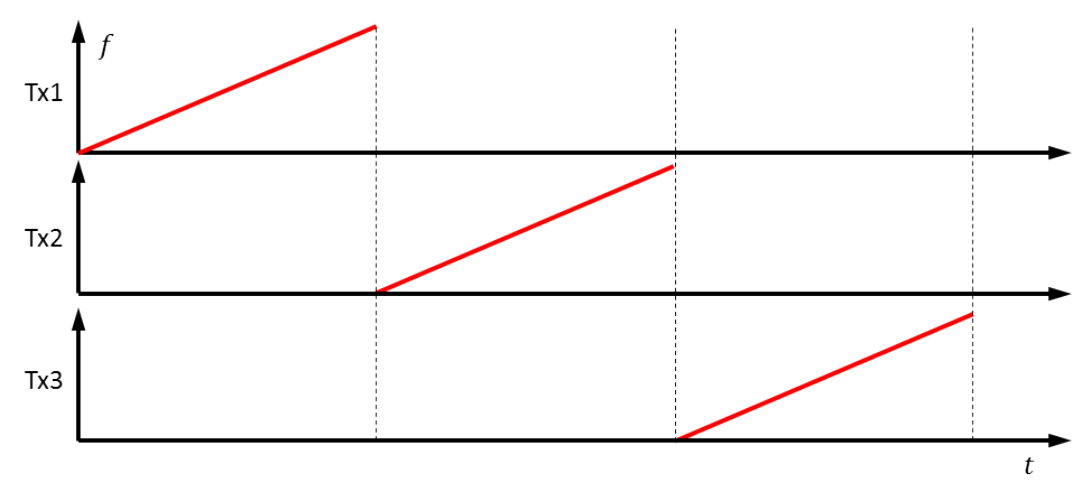

Figure 2.5: Time Division Multiplexing, each channel transmits chirp sequentially 


\subsubsection{MIMO ARRAY MODEL}

In FMCW MIMO radar systems, suppose the uniform linear array(ULA) has $M_{T}$ transmitters and $M_{R}$ receivers. The light of sight direction of the electromagnetic wave reflected from targets are assumed to be parallel, and thus the transmitted signals are delayed consecutively increasing to each element in an antenna array. Such delay includes both the time that the transmitted wavefront reaches the target and the received wavefront reaches the receiver element. In ULA, this phase shift is increasing linearly.

Suppose that the transmitted signals at each transmitter is $s_{i}$, where $i=1, \ldots, M_{T}$. A snapshot from all transmitters are sampled as $\mathbf{s}=\left[s_{1}, s_{2}, \ldots, s_{M_{T}}\right]$, where $\mathbf{s}_{i}$ are orthogonal to each other.

The steering vector, which indicates the phase delay of each element in antenna array, of transmitter $a_{T}$ and receiver $a_{R}$ are expressed as:

$$
\begin{aligned}
& \mathbf{a}_{T}(\theta)=\left[1, e^{j 2 \pi \frac{d_{T}}{\lambda} \sin (\theta)}, \ldots, e^{j 2\left(M_{T}-1\right) \pi \frac{d_{T}}{\lambda} \sin (\theta)}\right]^{T} \\
& \mathbf{a}_{R}(\theta)=\left[\left[1, e^{j 2 \pi \frac{d_{R}}{\lambda} \sin (\theta)}, \ldots, e^{j 2\left(M_{R}-1\right) \pi \frac{d_{R}}{\lambda} \sin (\theta)}\right]^{T},\right.
\end{aligned}
$$

where $d_{T}$ and $d_{R}$ represent the interelement spacing of the transmitter and receiver respectively. $\lambda$ is the wavelength of signals. The steering vector of virtual array is formulated through Kronecker product of transmitter and receiver array steering vectors:

$$
\mathbf{a}(\theta)=\mathbf{a}_{T}(\theta) \otimes \mathbf{a}_{R}(\theta) .
$$

To guarantee that there is only one main lobe in the beam pattern of virtual steering vectors, the distance between two virtual elements has to be at least $\lambda / 2$, as is shown in figure 2.6. To realize this spacing between virtual elements, we set the distance of transmitter element is $d_{T}=2 \lambda$ and the distance of receiver element is $d_{R}=\lambda / 2$.

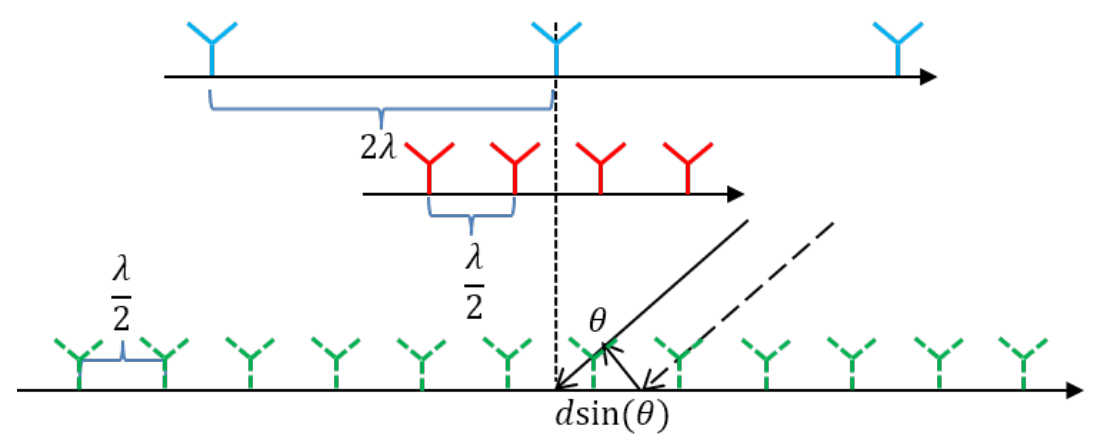

Figure 2.6: Virtual array with 12 elements, the number of transmitter and receiver are $M_{t}=3, M_{r}=4$. The blue solid antennas represent the transmitter elements, and the blue dashed antennas represent the virtual elements generated from red receiver antennas

Then the MIMO signal model can be expressed as

$$
\mathbf{x}=\mathbf{A B S}+\mathbf{n},
$$

where 


$$
\begin{aligned}
& \mathbf{A}=\left[\begin{array}{lll}
\mathbf{a}\left(\theta_{1}\right) & \cdots & \mathbf{a}\left(\theta_{k}\right)
\end{array}\right]^{H} \\
& \mathbf{B}=\left[\begin{array}{lll}
\beta_{1} & & \\
& \ddots & \\
& & \beta_{k}
\end{array}\right] \\
& \mathbf{S}=\left[\begin{array}{lll}
\mathbf{s}_{1} & \cdots & \mathbf{s}_{k}
\end{array}\right] \text {, }
\end{aligned}
$$

where $\mathbf{A}$ is the steering matrix, $\mathbf{B}$ is the amplitude of $k$ th target, $\mathbf{S}$ is the received signal matrix and $\mathbf{s}_{k}$ is the signal from the $k$ th target . The white noise $\mathbf{n}$ is assumed to be spatially independent and identically distributed. Consider the MIMO array model, 3D processing can be applied to the FMCW signals, which generates the output of range, Doppler, and angle information of targets. The model of signal data $\mathbf{X}$ of is shown in Figure 2.7

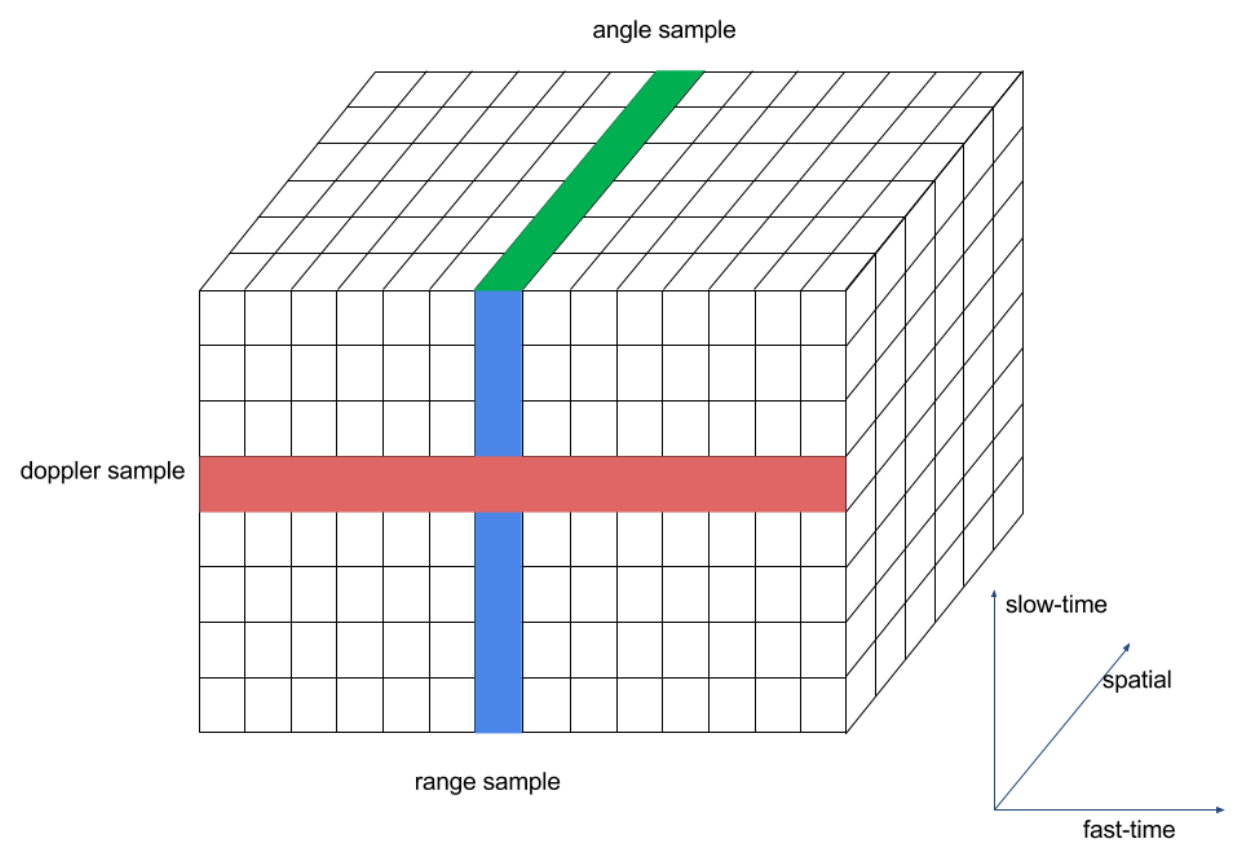

Figure 2.7: 3D data cube of FMCW MIMO signal model

\subsubsection{Review of Mimo DOA Estimation Algorithm}

The conventional DOA estimation in MIMO radar systems can be categorized into two types: non-parametric (spectral-based) methods and parametric methods [16]. The non-parametric algorithms (Bartlett, Capon, MUSIC and ESPRIT) exploit some spectrum-based function of the parameters to be estimated [17]. The parametric techniques, e.g., Deterministic and Stochastic Maximum Likelihood (DML and SML [18, 19]) algorithms have excellent DOA estimation performance. However, they require accurate initialization to guarantee the convergence of the algorithms and suffer from high computational complexity . 
The non-parametric methods include conventional digital beamforming and subspace-based algorithm. Although the subspace-based algorithm (MUSIC and ESPRIT) have very good performance on angular resolution, the main challenge of these super resolution DOA estimation algorithm is strongly correlated or coherent sources, the limited number of snapshots (sometimes even single snapshot), and the unknown number of targets.

Decorrelation algorithms such as spatial smoothing (SS) and forward-backward averaging (FBA) are used to restore the rank of the signal correlation matrix when signals are highly correlated or coherent. They make use of spatial-time data matrix symmetries such as shifting and centre symmetry [20].

In the limited number of snapshots scenario, adaptive algorithms (such as e.g., Capon, MUSIC, DML, and SML) that rely on an estimate of the noise covariance matrix cannot be applied [16, 21]. In FMCW automotive radar, in order to obtain the range-azimuth map, the only measurement that adjacent to the peak range cells of the target is available to estimate the covariance matrix, thus only one to three bins can be used for angle estimation, which is the main challenge of MUSIC[22].

Another challenge of MUSIC like the algorithm is that the unknown number of targets because it requires exact determination of effective rank of the correlation matrix. It is well known in literature that subspacebased techniques show the best performance at SNRs up to 20-25 dB, as is explained in [23], However, they show deteriorated performance in the SNR range below $5 \mathrm{~dB}$ when the number of targets is unknown [24].

In FMCW radar systems, beat signals convey range and angle information, which enables to estimate the azimuth, elevation, range, and velocity of unknown targets via joint parameter estimation techniques such as 2D-MUSIC, 2D-ESPRIT, JADE, and multidimensional Capon [24]. However, such multi-dimensional sub-space techniques increased the computational complexity quite a lot, which needs to be considered in a real-time FMCW automotive radar system.

\subsection{Monopulse Fundamentals}

Monopulse is an established technique for radars for fast and precise estimation of the direction of target. The monopulse technique is able to work with only one single data snapshot to realize DOA estimation, which is the reason to be named as 'mono' pulse [25]. Compared with other precise angle estimation methods, such as MUSIC, maximum likelihood (ML) estimation, it consumes much less computational time.

The monopulse radar utilizes received signals to generate sum and difference beams, which can be used to calculate monopulse ratio to accomplish accurate estimation of the direction of arrival. Conventional monopulse radar constructs error voltage from a two-antenna system, which receives signals separately to form sum and difference beam patterns.

Basically, monopulse radar can be divided into two types: amplitude comparison and phase comparison monopoulse radar. A conventional monopulse system for estimating one angle typically consists of two identical antennas, either separated by some distance (phase comparison monopulse, such as Figure 2.8 (a)) or at the same phase center but with a squint angle (amplitude comparison monopulse, as shown in Figure 2.8 (b)), whose outputs are summed up to produce a sum beam and are subtracted yielding the difference beam 
[26].
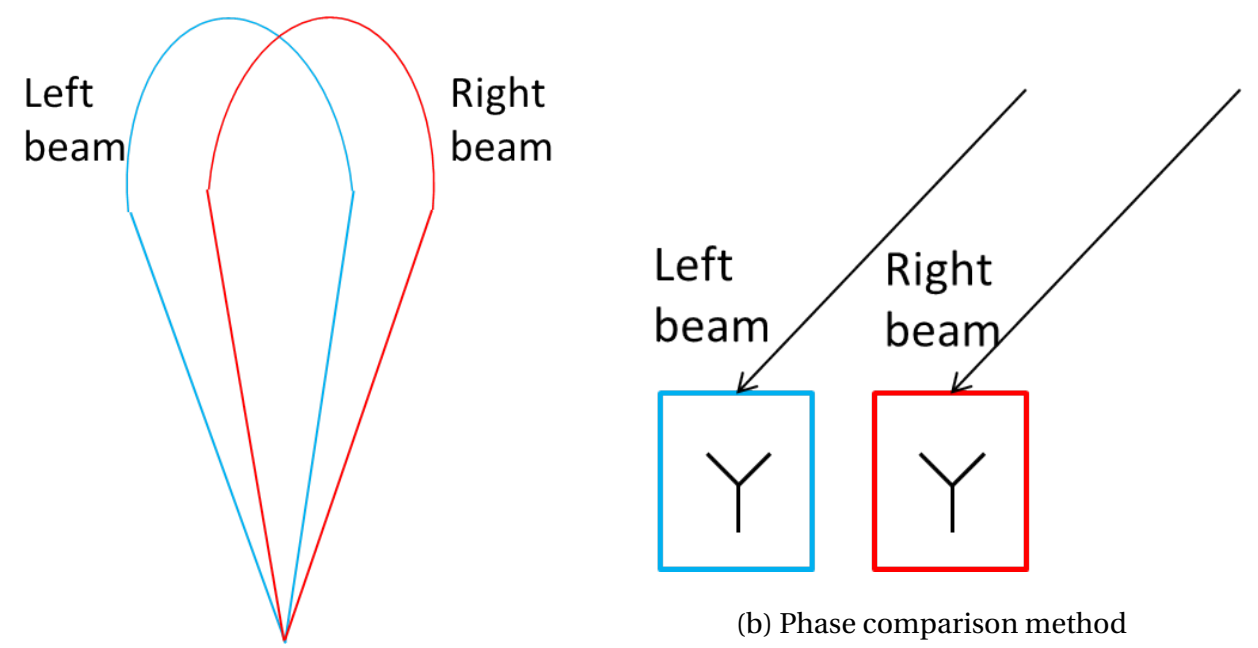

(b) Phase comparison method

(a) Amplitude comparison method

Figure 2.8: Monopulse classification: amplitude and phase comparison

The idea of amplitude comparison monopulse radar is to compare two receiving beams voltage simultaneously with an overlapping pattern on the main direction with a squint angle. The amplitude comparison monopulse considers only amplitude information, while the phase comparison monopulse radar measures the phase difference between two signals from the same target received by two different channels.

The state of art monopulse DOA estimation algorithms fully exploit standard monopulse and phased array monopulse, especially in tracking radar. In [27], standard monopulse is adopted for FMCW signals angle estimation on the application of hand gestures recognition. As for phased array monopulse, active electronically scanned array (AESA) is widely used and discussed in different publications. in the phased array monopulse radar is to optimize the sum and difference pattern using subarray configuration with reduced complexity of beamforming-network (BFN) [28, 29]. Adaptive monopulse and space-time adaptive processing is also considered in [30-32].

The application of monopulse in MIMO distributed radar has been published in [33][34]. Widely separated MIMO with distributed array using Capon beamformer to generate amplitude comparison sum and difference channel are utilized for tracking application through data fusion. Monopulse combined with colocated MIMO with amplitude and phase comparison monopulse is also discussed in literature [35-37], but none of them consider FMCW signals. Also, they didn't utilize any synthesis of sum and difference beam which can improve the performance of angle estimation using MIMO-monopulse. Moreover, monopulse ratio ambiguity is not discussed, which is a big issue for MIMO-monopulse algorithm. 



\section{3 \\ DOA ESTIMATION USING MIMO-MONOPULSE}

In this chapter the conventional monopulse algorithm will be extended to MIMO radar. Since MIMO-monopulse utilizes antenna array to generate sum and difference beam, firstly we will discuss the details of digital beamforming technique of MIMO array. The conventional method of generating sum and difference beam will be introduced. Then we propose a synthesis of sum and difference beam to improve the performance of MIMO-monopulse.

\subsection{Digital BeAmforming ( DBF)}

Beamforming, also known as spatial filtering, is a kind of technology that focus the radar beam of antenna array to some desired direction over azimuth or elevations. In an antenna array, beamforming is realized through summing up the weighted signals of each elements, the weighting basically includes information of amplitude and phase. As a result, signals at a specific direction will be constructively interfered and thus a narrow beam will be directed to the angle of interest.

\subsubsection{NARROW BAND BEAMFORMING}

With narrow-band systems the time delay is approximated as a "phase shift". A narrow band radar systems is one where the bandwidth is only a small fraction of the center frequency. With wide band systems this approximation no longer holds. The definition of narrow band is [38, 39]

$$
B(M-1) \frac{d}{c} \ll 1,
$$


where $B$ is the bandwidth of the signal, $M$ is the number of the element, and $d$ is the inter-element spacing. If 3.1 is not satisified, the signal cannot assumed to be narrow band and the beam pattern becomes a twodimensional function (see Section 3.1.2).

Conventional beamformers use a fixed set of weightings and time-delays to combine the signals from the sensors in the array, primarily using only information about the location of the sensors in space and the wave directions of interest. Advanced algorithms called adaptive beamforming techniques generally combine this information with properties of the signals actually received by the array, typically to improve rejection of unwanted signals from other directions (see in Section 4.2.1). This process may be carried out in either the time or the frequency domain.

In phased array radar, beamforming is normally implemented through analog phase shifter which can alter the phase electronically, thus steering the beam of radio waves to a different direction. In MIMO radar systems, digital beamforming ( DBF) can be realized in virtual element level by modifying the weighting (amplitude and more importantly phase) of signals received by each channel.

Consider the uniform linear array (ULA) MIMO radar which has $M_{T}$ transmitters and $M_{R}$ receivers. The virtual array steering vector is defined as the Kronecker product of transmitting and receiving arrays steering vectors, which is equivalent to the physical receiving channels of radar and can be directly processed to achieve beamforming. For a ULA with $M_{T}$ transmitters and $M_{R}$ receivers, there will be $M_{T} \times M_{R}$ virtual elements in the array. In beamforming, we estimate the signal of interest arriving from a specific direction. The output of beamforming can be expressed as

$$
y=\mathbf{w}^{H} \mathbf{x}
$$

where

$$
\mathbf{w}=\left[\begin{array}{c}
w_{1} \\
w_{2} \\
\vdots \\
w_{M}
\end{array}\right], \quad \mathbf{x}=\left[\begin{array}{c}
x_{1} \\
x_{2} \\
\vdots \\
x_{M}
\end{array}\right] .
$$

and $M$ is the number of channels, namele $M_{T} \times M_{R}$. The weighting vector $\mathbf{w}$ can be determined through some beamforming algorithms (such as Bartlett or Capon). Without losing generality, consider a complex plane wave signal $x(t)=e^{j 2 \pi f_{c} t}$ with center frequency $f_{c}$ and a DOA angle $\theta_{0}$. Under far-field assumptions, the wavefront is assumed to be planar, thus the line of sight from each element to the target can be seen as parallel and the time delays for each consecutive element is linearly increasing in the ULA. Thus the signal received by first sensor will be $x_{0}(t)=x(t)$ and by $m$ th sensor will be $x_{m}(t)=x\left(t-\tau_{m}\right)=e^{j 2 \pi f_{c}\left(t-\tau_{m}\right)}$. Then the output of beamformer can be writter as

$$
y=e^{j 2 \pi f_{c} t} \mathbf{w}^{H}\left[\begin{array}{lllll}
e^{-j 2 \pi f_{c} \tau_{0}} & e^{-j 2 \pi f_{c} \tau_{1}} & e^{-j 2 \pi f_{c} \tau_{2}} & \cdots & e^{-j 2 \pi f_{c} \tau_{m}}
\end{array}\right]^{T} .
$$

With $\tau_{0}=0$, define the response of beamformer as

$$
P\left(\theta_{0}\right)=\mathbf{w}^{H}\left[\begin{array}{lllll}
e^{-j 2 \pi f_{c} \tau_{0}} & e^{-j 2 \pi f_{c} \tau_{1}} & e^{-j 2 \pi f_{c} \tau_{2}} & \cdots & e^{-j 2 \pi f_{c} \tau_{m}}
\end{array}\right]^{T}=\mathbf{w}^{H} \mathbf{a}\left(\theta_{0}\right),
$$


where $\mathbf{a}$ is the steering vector (See equation 2.15). The time delay $\tau_{m}$ can be calculated as $m d / \lambda \sin \theta, m=$ $1,2, \ldots, M-1$, where $d$ is the inter element spacing in ULA and suppose the broadside direction is at $0^{\circ}$. The response beam pattern of digital beamforming is shown in Figure 3.1.

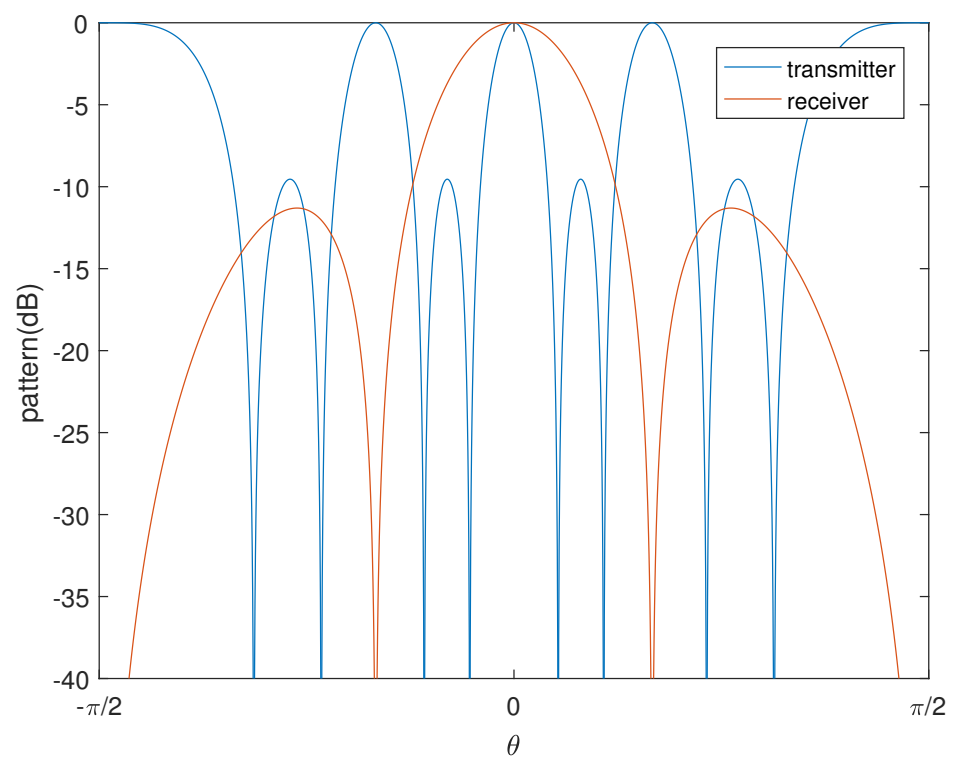

(a) Transmitter (blue) and receiver (red) array

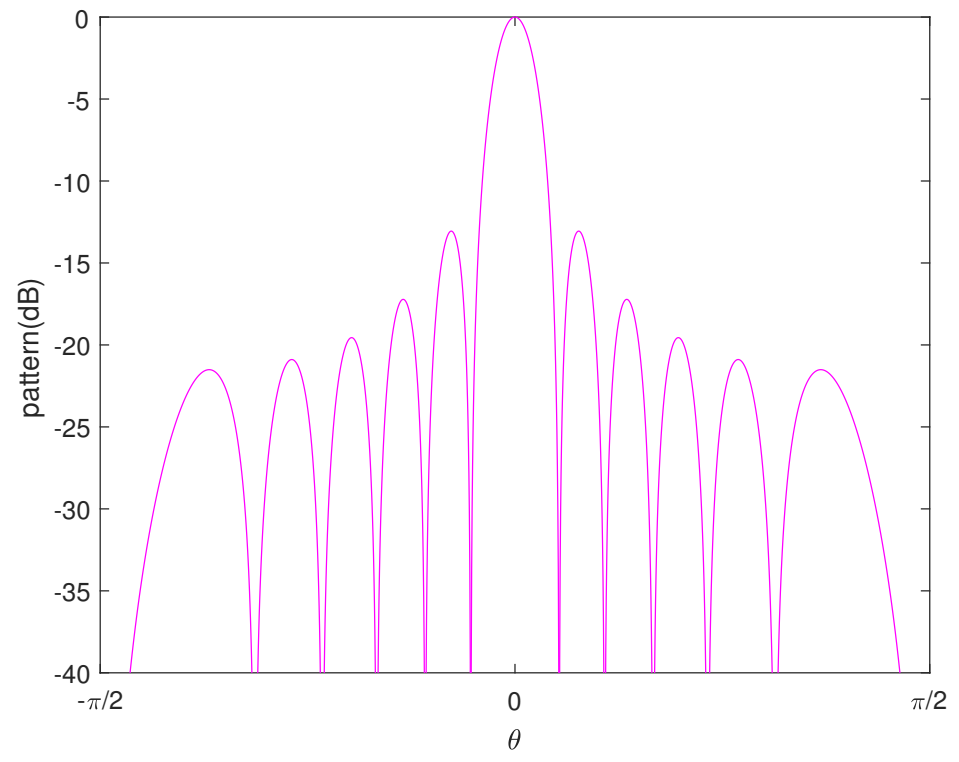

(b) Virtual array

Figure 3.1: Digital beamforming response pattern

\subsubsection{The EFfect of Wideband}

The beamforming structure introduced previously works effectively only for narrowband signals. When the signal bandwidth increases, its performance will degrade significantly. In wideband scenario, the weighting vector is not not only the function of DOA, but also the function of the frequency, since the wavelength of 
different frequency component is no longer fixed. If we still use the fixed weighting vector, the dispersion occurs that the beam is steered with a direction offset as is shown in Figure 3.3. Consider a wideband signal with bandwidth $B$, after modulation the frequency of the signal will be from $f_{c}-B / 2$ to $f_{c}+B / 2$. The element spacing of virtual array should be chosen as $\lambda=c /\left(f_{c}+B / 2\right)$ to guarantee no grating lobe. The steering vector has become

$$
\mathbf{a}(\theta, f)=\left[\begin{array}{llll}
1 & e^{j 2 \pi f\left(\frac{d}{c}\left(f_{c}+\frac{B}{2}\right) \sin \theta\right.} & \cdots & e^{j 2 \pi f(M-1) \frac{d}{c}\left(f_{c}+\frac{B}{2}\right) \sin \theta}
\end{array}\right]^{T},
$$

where $f=\left[f_{c}-B / 2, f_{c}+B / 2\right]$. Therefore the wideband pattern response also turns to a function of frequency $P(\theta, f)=\mathbf{w}^{H} \mathbf{a}(\theta, f)$, which the width of the main beam and the position of nulling both changes with center frequency. If we still choose the beamformer weighting vector like what we did for narrowband beamforming as $\mathbf{w}\left(\theta_{0}, f_{c}+B / 2\right)$, the direction of beam will not be steered to the same angle for varying frequency, which is called the effect of frequency dispersion, as is shown in Figure 3.3. In this figure, the estimation error is quite obvious when the center frequency are $77 \mathrm{GHz}$ and $81 \mathrm{GHz}$, which covers the full $4 \mathrm{GHz}$ bandwidth of short range automotive radar application. In actual radar chip it may not utilize the full bandwidth which doesn't have such dramatic effect on estimation accuracy, but as a scientific research we still study the effect of bandwidth, since the concept of MIMO monopulse FMCW radar is also possible applied on other wideband radar.

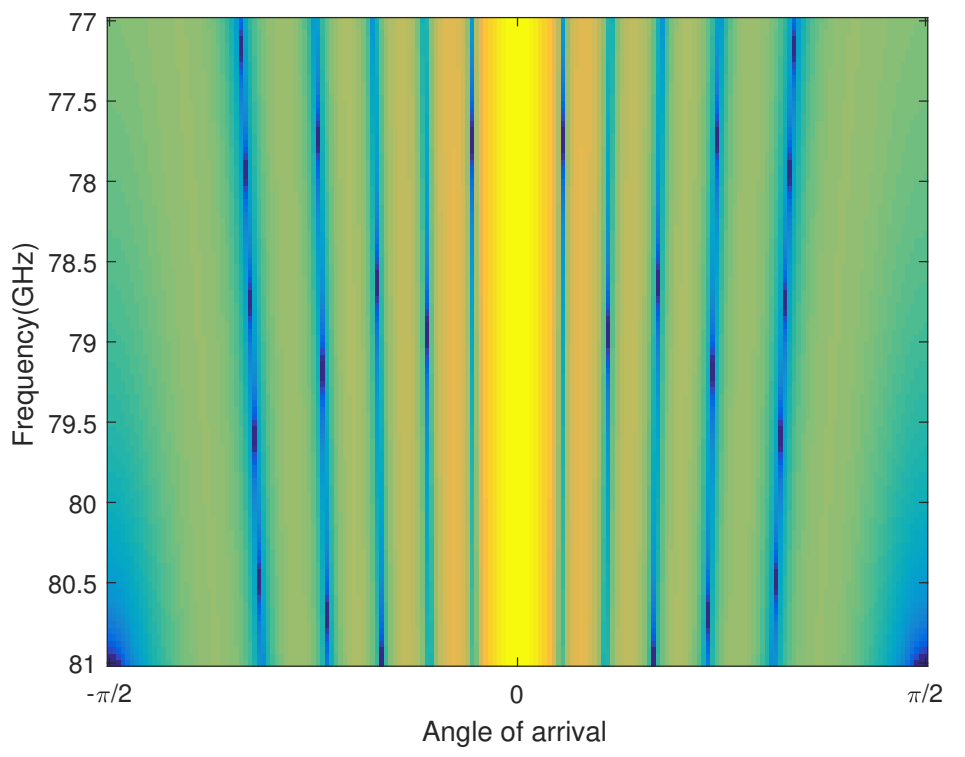

Figure 3.2: Wideband DBF response pattern, beam width decreases with frequency increasing

For wideband beamforming, the value of the weights should be adjusted for different frequencies. Traditionally, an easy way to form such a set of frequency dependent weights is to use a series of tapped delay-lines (TDLs), or FIR/IIR filters in its discrete form[40]. Both TDLs and FIR/IIR filters perform a temporal filtering process to form a frequency dependent response for each of the received wideband sensor signals to compensate the phase difference for different frequency components. The beamformer obeying this architecture samples the wideband signals in both space and time. The output of such a wideband beamformer can be 
expressed as:

$$
y=\sum_{i=0}^{J-1} \mathbf{w}_{i}{ }^{H} \mathbf{x}\left(t-i T_{s}\right)
$$

where $T_{s}$ is the sampling frequency of the system and should no more than $\left.T_{\min }=\frac{c}{2}\left(f_{c}+B / 2\right)\right)$. In each tap $i$, the weighting vector can be determined through the new steering vector:

$$
\mathbf{a}(\theta, f)=\left[\begin{array}{cccc}
1 & e^{j 2 \pi f \tau_{1}} & \cdots & e^{j 2 \pi f \tau_{M}} \\
\vdots & & & \\
1 & e^{j 2 \pi f\left[\left(\tau_{1}+(J-1) T s\right]\right.} & \cdots & e^{j 2 \pi f\left[\tau_{M}+(J-1) T s\right]}
\end{array}\right]^{T} .
$$

The required steering delay can be realized by fractional delay filter.

Another option for wideband beamforming is to decompose the spatial channels into a number of subbands and then perform narrow beamforming separately in each subband [41], which has a computational advatage compared to TDLs.

In this thesis, the wideband signal is linearly frequency modulated, which has the property that the center frequency of chirp signals changes by time with a exact time-frequency mapping (coupling between time and frequency). Thus we can process the wideband FMCW signals using a time-varying weighting vector, which is expressed as

$$
\mathbf{w}\left(\theta_{0}, t\right)=\left[\begin{array}{llll}
1 & e^{j 2 \pi f\left(\frac{d}{c}\left(f_{c}-\frac{B}{2}+\mu t\right) \sin _{0} \theta\right.} & \cdots & e^{j 2 \pi f(M-1) \frac{d}{c}\left(f_{c}-\frac{B}{2}+\mu t\right) \sin _{0} \theta}
\end{array}\right]^{T},
$$

where $t$ in digital sampling is taken as $n T_{s}$ within the interval of a chirp duration. The result of time varyingweighting is shown in Figure 3.3 (b). Notice that although the direction of main beam is steered correctly, the beam width of two response is different and the nulling of pattern is quite a lot shifted, which has effect if an adaptive beamforming is applied for interference cancellation.

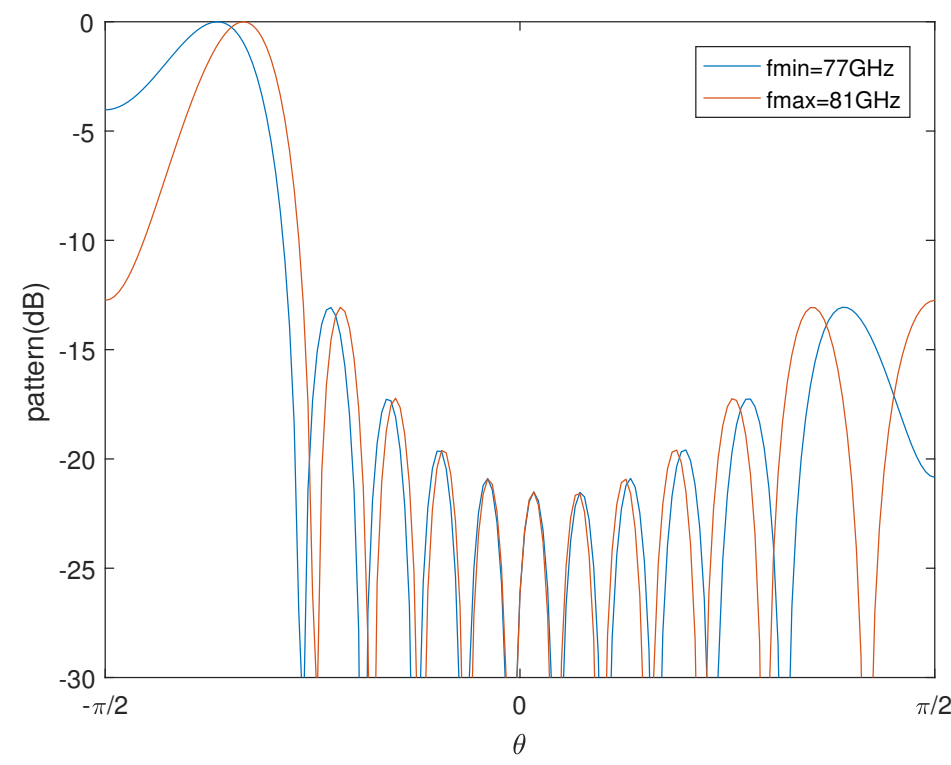

(a) Frequency dispersion using fixed weighting, target is at $-60^{\circ}$ 


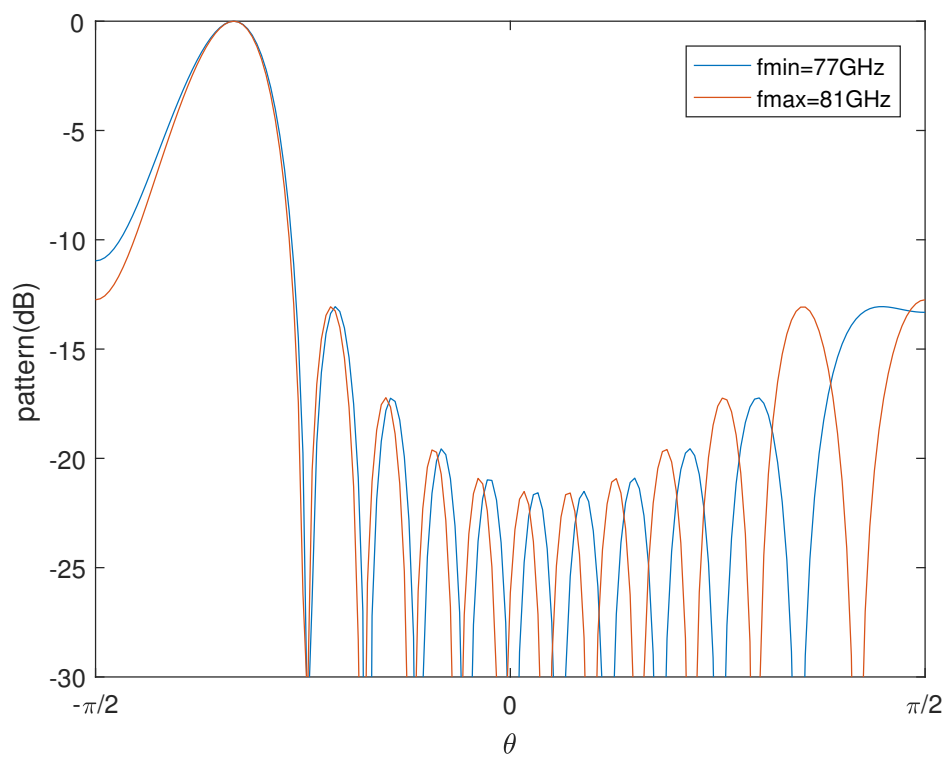

(b) Time-varying weighting

Figure 3.3: Wideband beamforming, the beam is steered to $-60^{\circ}$

\subsection{MimO Monopulse}

The MIMO monopulse radar systems use virtual array to generate sum and difference pattern beams, which is very similar to the case of phased array monopulse. Basically both amplitude and phase comparison monopulse utilize beamforming algorithm to calculate sum and difference beam. The DOA is determined through the ratio of the sum to difference beam as error voltage.

\subsubsection{AMPLITUDE COMPARISON}

For amplitude comparison monopulse, the most straightforward strategy is to consider the sum and difference channels to be the sum and difference of two beamforming vectors centered about boresight $\theta_{0}$ but separated by a squint angle shift $\theta_{s}$ to left and right [42]. There is a trade off of choosing the value of squint angle, because a small squint angle leads to a sharp monopulse ratio, but the linear region will be too narrow for tracking a moving target. However, a large value of squint angle will generate a distortion sum beam pattern, and usually the squint angle is chosen as the $-3 \mathrm{~dB}$ point of the beam [43]. But in our simulation we found the squint angle should be smaller in order to get obtain a ideal sum beam pattern, since -3dB point still caused too widely separated left and right beams and will distort the sum beam. Consider a MIMO virtual array of $M$ elements, given the steering vector defined in (11), define the weights of two beamformers as $\mathbf{w}_{l}$ and $\mathbf{w}_{r}$, where the subscripts $l$ and $r$ correspond to the left and the right beams, respectively. Then, the amplitude comparison beamforming weights are given by:

$$
\begin{aligned}
& \mathbf{w}_{\mathbf{l}}=\mathbf{a}\left(\theta_{0}-\theta_{s} / 2\right) \\
& \mathbf{w}_{\mathbf{r}}=\mathbf{a}\left(\theta_{0}+\theta_{s} / 2\right),
\end{aligned}
$$




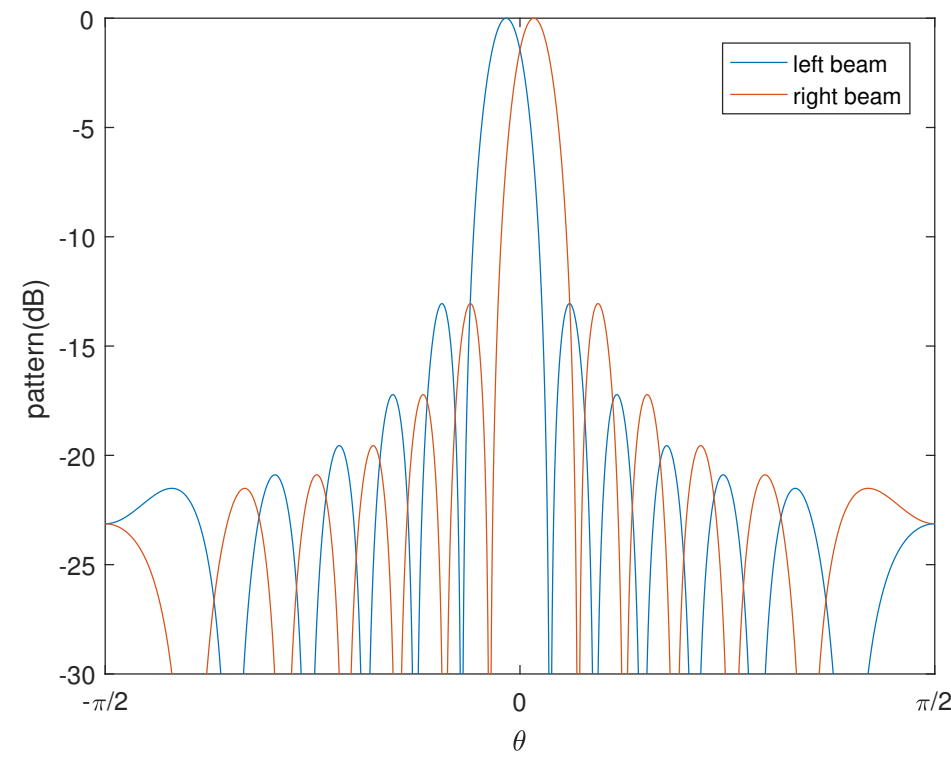

Figure 3.4: Left beam and right beam of amplitude comparison monopulse

where $\mathbf{w}_{\mathbf{l}}$ and $\mathbf{w}_{\mathbf{r}}$ are Bartlett beamforming vectors, but it can also be Capon beamforming vectors [33]. In [44], several methods of robust adaptive monopulse beamforming techniques were explored to solve interference. The DBF response pattern of left and right beams are shown in Figure 3.4. Therefore, the sum and difference weight vectors are respectively expressed as:

$$
\begin{aligned}
& \mathbf{w}_{\Delta}=\mathbf{w}_{l}-\mathbf{w}_{r} \\
& \mathbf{w}_{\Sigma}=\mathbf{w}_{l}+\mathbf{w}_{r} .
\end{aligned}
$$

Then the monopulse ratio is defined by the ratio of difference to sum output. If the radar scans over a target, the sum beam output will be maximum when the target is exactly in the look direction $\theta_{0}$, and the difference beam output will then be zero, thus the monopulse ratio will be exactly zero. If the target has a small offset angle from the look direction $\theta-\theta_{0}$ the small output of the difference beam, the "error voltage" is used to estimate this angle, which is defined as the real part of monopulse ratio [32].

$$
\epsilon=\Re\left\{\frac{\mathbf{w}_{\Delta}^{H} x}{\mathbf{w}_{\Sigma}^{H} x}\right\},
$$

where $x$ is the signal of interest. Once an error voltage is available, it can be used in conjunction with a monopulse response curve (MRC) to arrive at an angle estimate $\hat{\theta}$ of the target's true azimuth $\theta$. The MRC is defined as the real part of the ratio of difference to sum responses with which the $\hat{\theta}$ is estimated. Then the angle is estimated through inverse mapping $\epsilon^{-1}$ to the error voltage:

$$
\hat{\theta}=\epsilon^{-1}\left(\Re\left\{\frac{\mathbf{w}_{\Delta}^{H} \mathbf{a}(\theta)}{\mathbf{w}_{\Sigma}^{H} \mathbf{a}(\theta)}\right\}\right) .
$$

Given an error voltage measurement, the target azimuth is determined by inverse mapping the error voltage through the MRC, which is shown in Figure 3.6. 


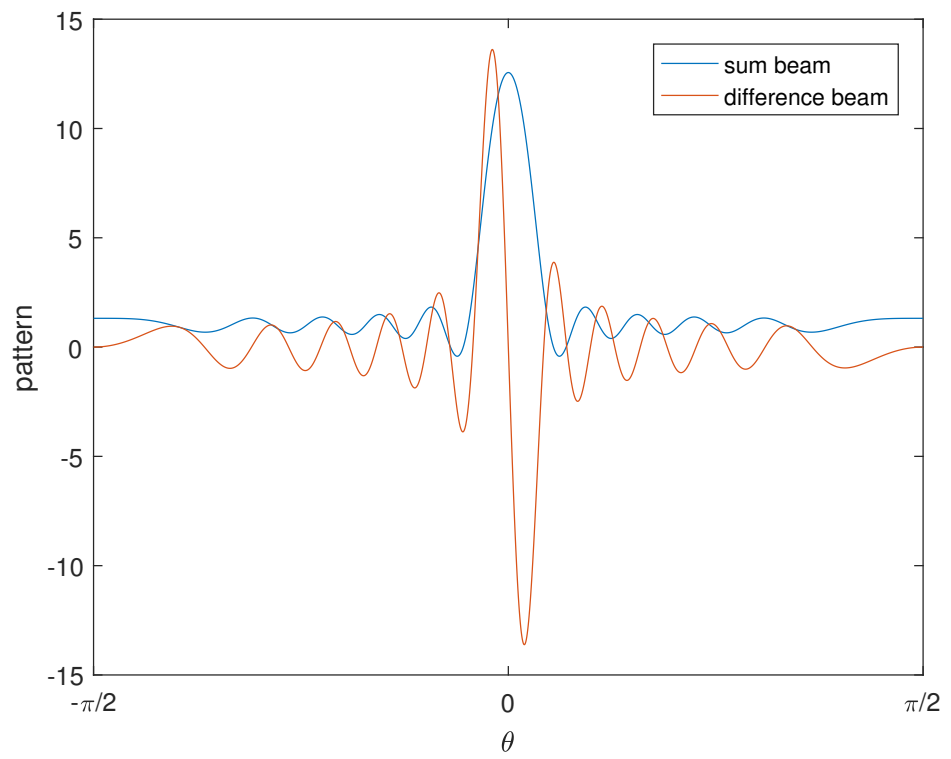

(a) Real part of sum and difference beam

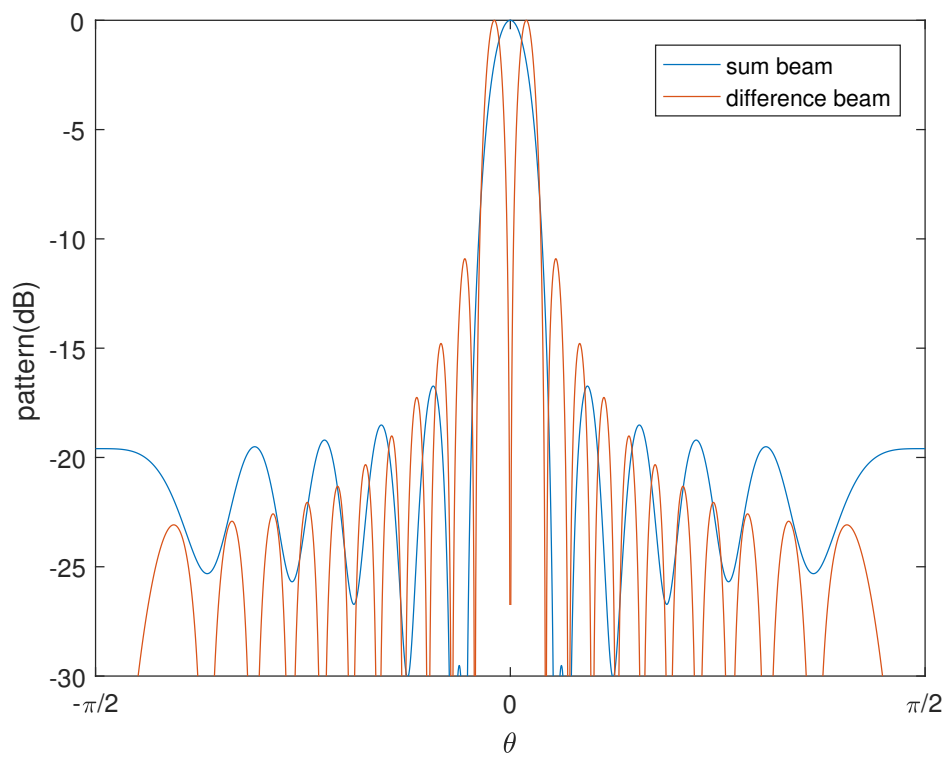

(b) Sum and difference beam pattern in $\mathrm{dB}$

Figure 3.5: Sum and difference beam of amplitude comparison monopulse

The real part of sum and difference beam is shown in Figure 3.5 (a), and the beam patterns are shown in Figure 3.5 (b).

And there are also half phase reserved and derivative method [45] for amplitude comparison. The half phase reserved method generates the right beam weighting through multiplying the left beam weighting with imaginary number $-i$, and the derivative method directly generate difference beam pattern by taking derivative of sum beam pattern. 


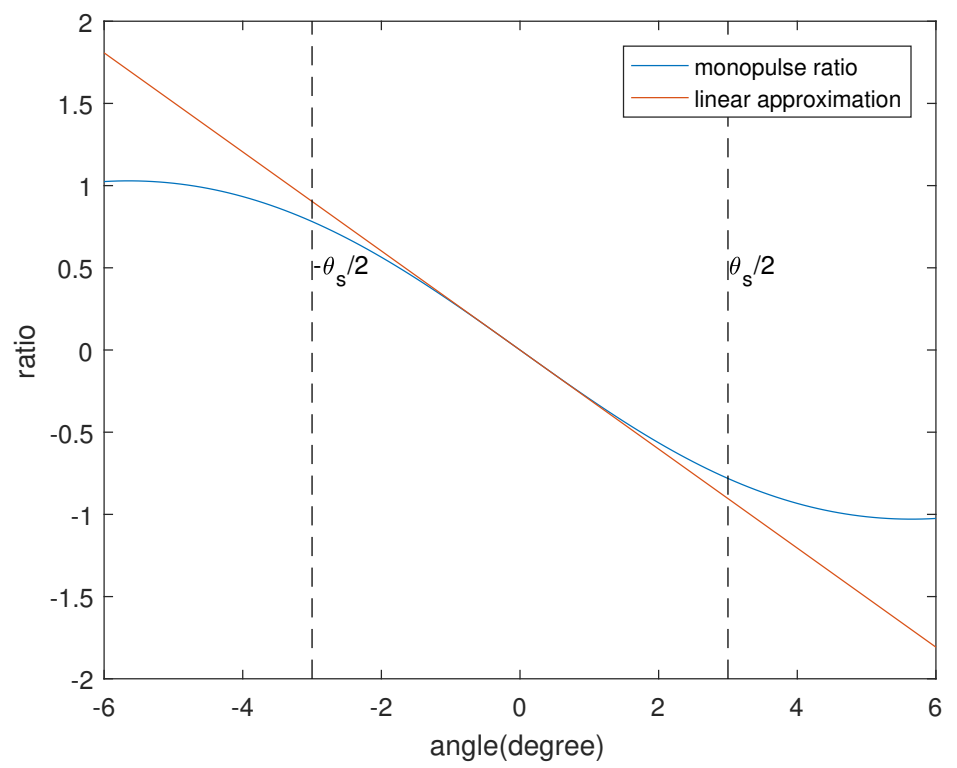

(a) Error voltage and inverse mapping (zoomed)

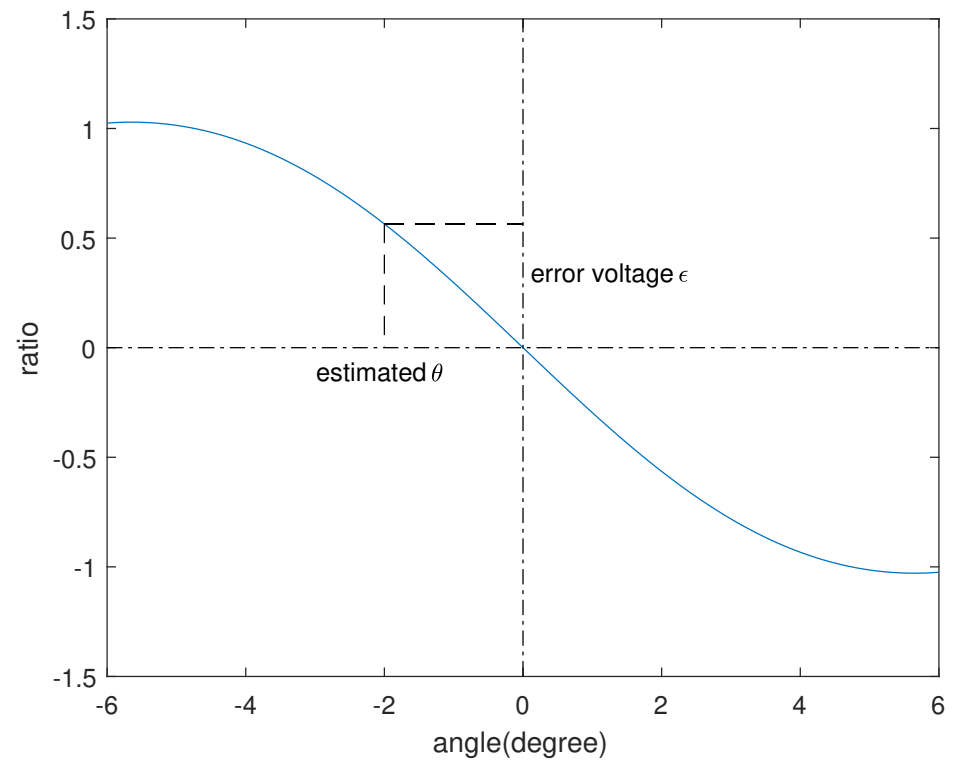

(b) Real part of sum and difference beam (zoomed)

Figure 3.6: Monopulse response curve (MRC)

\subsubsection{PHASE COMPARISON}

If we consider the phase information of the beam, virtual array can divided into two sub-arrays averagely when applying phase comparison angle monopulse. Unlike amplitude comparison, the left and right beam forming vector weights are defined for two sub-array respectively. Consider a virtual array of $M$ elements, the left $M / 2$ elements consists of left sub-array while the rest of elements consists of the right one. Left and right beams are generated by two sub-array separately, and define $\mathbf{w}_{l}$ and $\mathbf{w}_{r}$ as the left and right beamforming vectors, which both have length of $M / 2$. The steering vectors of two sub-array are expressed as $\mathbf{a}_{l}$ and $\mathbf{a}_{r}$. The 
left beam and right beam pattern have very close amplitude at the looking direction, but the phase between two sub arrays is different, which is utilized to determine the angle of the target. Only when the target is exactly located at the direction of the beam, the phase difference between left beam and right beam is 0 , as is illustrated in Figure 3.7. The region within $3 \mathrm{~dB}$ width of sum beam is shown as well.

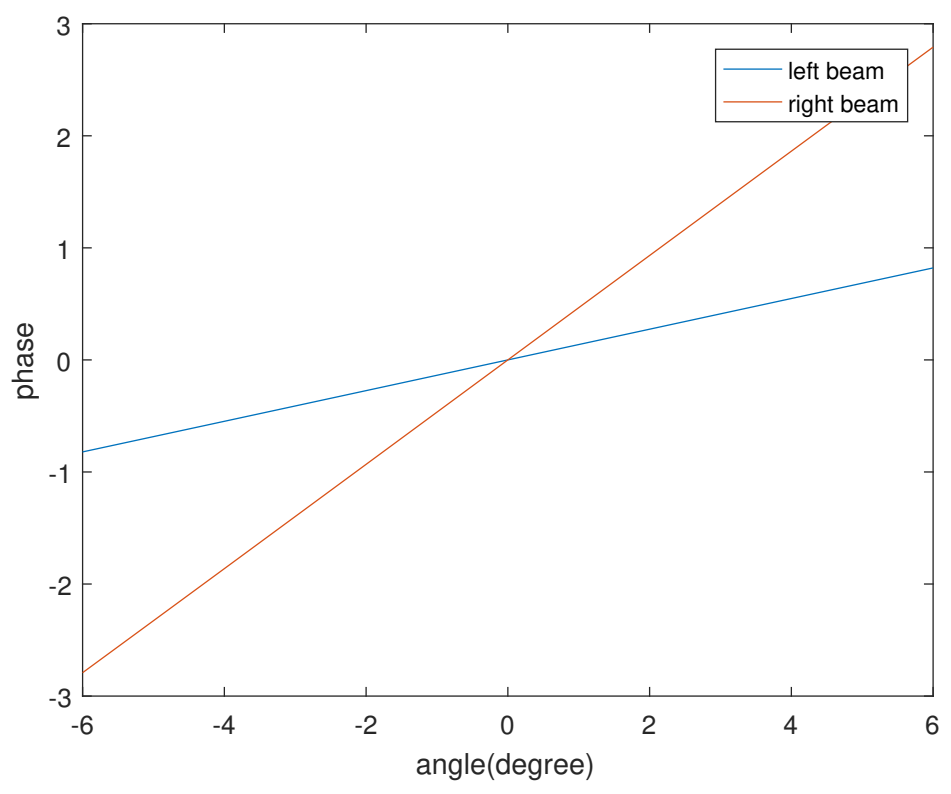

Figure 3.7: Phase of left beam and right beam (zoomed)

Thus the sum and difference pattern of phase comparison monopulse are defined as [37]:

$$
\begin{gathered}
P_{\Delta}(\theta)=\mathbf{w}_{l}^{H} \mathbf{a}_{l}(\theta)-\mathbf{w}_{r}^{H} \mathbf{a}_{r}(\theta) \\
P_{\Sigma}(\theta)=\mathbf{w}_{l}^{H} \mathbf{a}_{l}(\theta)+\mathbf{w}_{r}^{H} \mathbf{a}_{r}(\theta),
\end{gathered}
$$

where $\mathbf{w}_{l}=\mathbf{a}_{l}\left(\theta_{0}\right)$ and $\mathbf{w}_{r}=\mathbf{a}_{r}\left(\theta_{0}\right)$. The sum and difference pattern is shown in Figure 3.8 (a).

The monopulse response curve is still expressed as $q(\theta)=\frac{P_{\Delta}}{P_{\Sigma}}$. Compared with amplitude comparison monopulse, one difference is that in phase comparison monopulse we take imaginary part of monopulse ratio as error voltage. The imaginary of phase comparison monopulse ratio is a tangent function, which is also approximated to be linear in a local region around the looking direction[26]. 


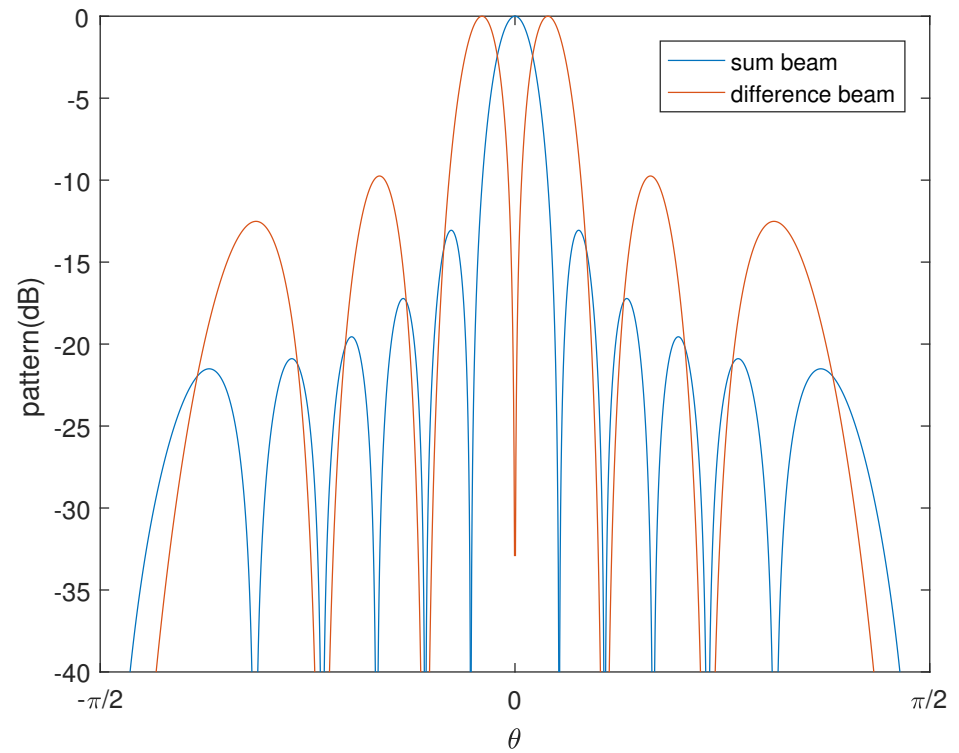

(a) Beam pattern

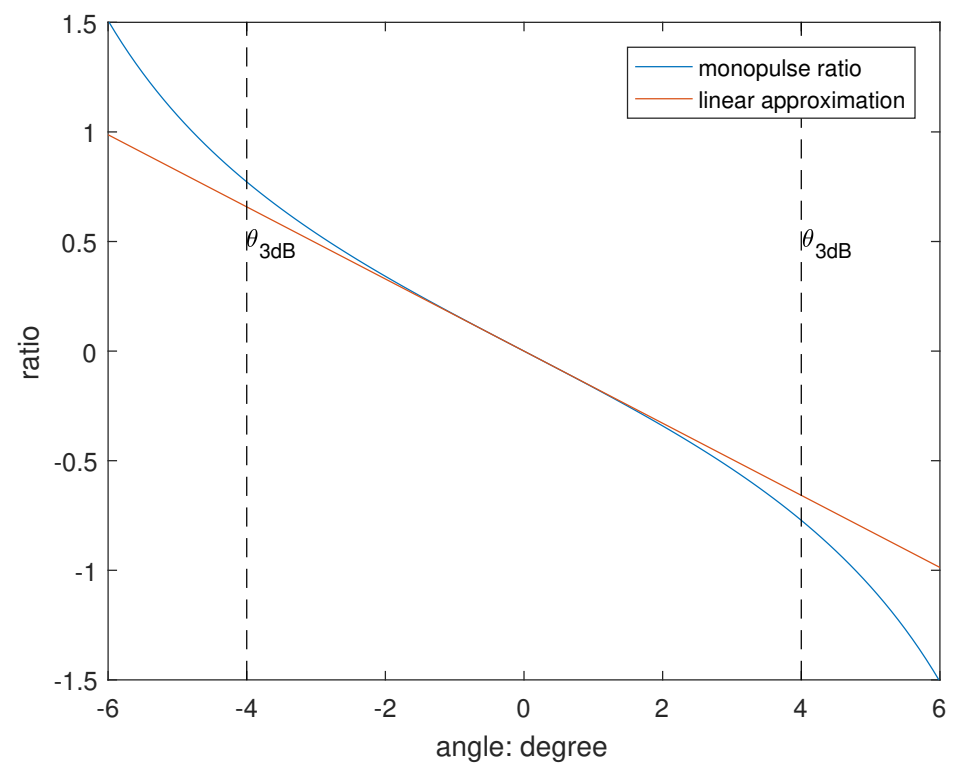

(b) MRC using phase comparison monopulse

Figure 3.8: Sum and difference pattern, monopulse ratio using phase comparison method

\subsection{Synthesis of Sum And Difference Pattern}

MIMO-monopulse radar utilizes sum and difference pattern to obtain accurate angle estimation, which can be further improved through synthesis of array pattern. The goal of synthesis of array is to obtain the beam pattern with desired property through choosing the weighting of each element. Such property includes narrow beamwidth, low side lobe level (SLL) and high directivity. In monopulse application, the constraints of sum and difference pattern should be separately considered. As far as the sum pattern is concerned, there is the need of maximizing the gain. On the other hand, the more critical issues to be addressed dealing with 
difference patterns are concerned with both the first null beamwidth and the normalized difference slope on boresight direction, since they are strongly related to the sensitivity of the radar (i.e., to the angular error).

\subsubsection{Dolph-CHebyshev Sum PATTERN}

The Dolph-Chebyshev synthesis [46] of linear arrays with sum patterns gives the array element excitations that provide an array factor which is optimum in the sense that for a specified sidelobe level, number of array elements, and interelement spacing, the beamwidth between the first nulls is the narrowest possible. It provides the minimum null-to null main-lobe width, for a given sidelobe level, or minimum sidelobe level for a given null-to-null main-lobe width. The Dolph-Chebyshev beampattern control the beamwidth and sidelobe level through the property of Chebyshev polynomials. The beampattern is of the form:

$$
B(\theta)=\frac{1}{H} T_{\alpha}(k \cos (\theta / 2)),
$$

where $\theta$ is the angle, $T_{\alpha}$ is the Chebyshev polynomial of order $\alpha, H$ is the ratio between the maximum mainlobe level and the maximum sidelobe level, and $k$ is a parameter controlling the null-to-null beamwidth $2 \theta_{0}$, given by,

$$
k=\frac{\cos \left(\frac{\pi}{2 M}\right)}{\cos \left(\theta_{0} / 2\right)},
$$

such that

$$
\theta_{0}=2 \cos ^{-1}\left(\frac{1}{k} \cos \left(\frac{\pi}{2 \alpha}\right)\right) .
$$

The maximum main lobe amplitude is one and the maximum sidelobe amplitude is $1 / H$. Chebyshev beampattern of order $M$ can be realized through two methods. Firstly, sidelobe level $1 / H$ is specified and then the parameter $k$ is given by

$$
k=\cosh \left(\frac{1}{\alpha} \cosh ^{-1}(H)\right) .
$$

Alternatively, the desired beamwidth $2 \theta_{0}$ is specified, from which $k$ is calculated using 3.17. Then, $H$ is calculated using

$$
H=\cosh \left(\alpha \cosh ^{-1}(k)\right) .
$$

Once $H$ and $k$ are available, the resulting beampattern is given by 3.15 .

To realize the Chebyshev beam pattern in DBF, the normal steering vector is multiplied with a taper, which is a window vector with amplitude excitation coefficients (This procedure is called shading in antenna design). The determination of coefficients can be found in paper [47], or realized through inverse discrete fourier transform of a Chebyshev window in frequency domain, which is described in [48]. 


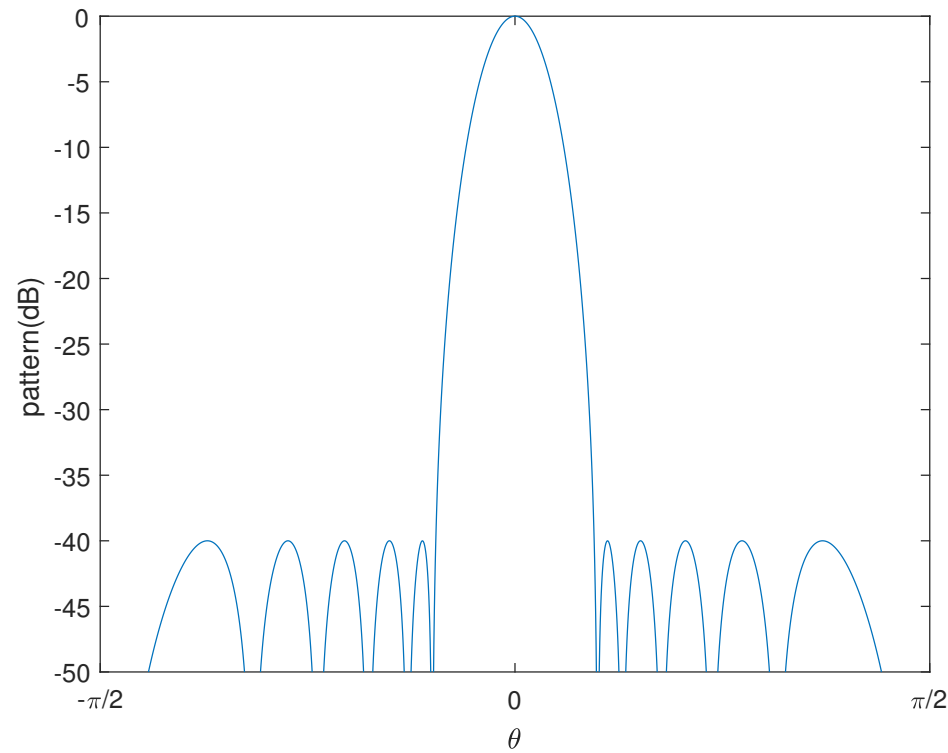

(a) Beam pattern

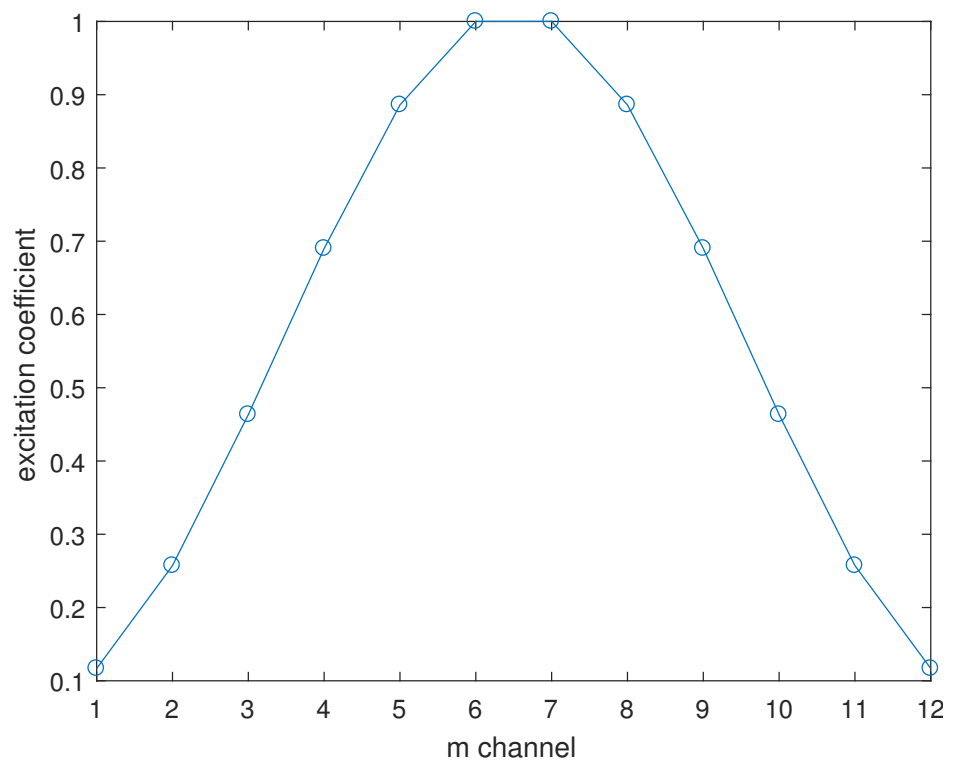

(b) Excitation Coefficients

Figure 3.9: Dolph-Chebyshev sythesis of sum pattern of 12 elements, sidelobe level is $-40 \mathrm{~dB}$

\subsubsection{Zolotarev Difference Pattern}

A difference pattern can be defined as optimum in the Dolph- Chebyshev sense if it has the narrowest first null beamwidth and largest normalised difference slope on boresight for a specified sidelobe level. Such patterns have sidelobes all at the same required level. Price and Hyneman [49] demonstrated that linear array difference patterns with equal sidelobe levels are ideal, i.e. they display the narrowest main difference lobe width and the largest slope on boresight for a specified sidelobe level. They subsequently concluded that no known polynomial, which could be used to find the weights to be applied to each element, had the required 
characteristics to generate this pattern. McNamara [50] subsequently realised that a class of functions known as Zolotarev polynomials did possess exactly the properties required for ideal linear array difference beam synthesis. They have characteristics similar to the Dolph-Chebyshev sum beam.

Implementation of the Zolotarev polynomial requires knowledge of elliptic integrals, Jacobi moduli, and Jacobi eta, zeta and elliptic functions. McNamara has also extended his work to allow for the creation of modified-Zolotarev linear array difference patterns, and the application of Zolotarev methods to planar arrays. In paper [51], a close approximation to the Zolotarev funtion, that is easy to implement, has been developed for the linear array case.

The general form of a continuous aperture difference beam weight function $g(z)$ can be written as follows, where $z$ is the normalised distance from the centre of the linear array, i.e. $-1 \leq z \leq 1$ :

$$
g(z)=f(z) \times(\text { sum beam weighting expression of } z),
$$

where $f(z)$ is an antisymmetric function of $z$. Then the final beam pattern of an antenna array will be the entrywise product of an antisymmetric weighting and a sum beam weighting. The sum beam is DolphChebshev pattern in this case to results in all sidelobes being equal. In [51] the author found that using $f(x)=x$ or $f(x)=\sin (x \times \pi / 2)$ will yields the difference patterns with first few sidelobes either too high $(x)$ or too low $(\sin x)$. Thus an antisymmetric function suggested is to make use of the Taylor expansion of $\sin x$ in $x$

$$
f(x)=x-\frac{a}{3} x^{3}
$$

where $a$ is a constant, which should be a suitable value for an antisymmetric function between $x$ and $\sin x$ that better approximates the desired sidelobe levels. According to [51], the approximation of Zolotarev difference pattern has $30 \mathrm{~dB}$ sidelobe corresponding to the Chebyshev pattern (used in equation 3.20) with sidelobe level of $40 \mathrm{~dB}$. The approximation of difference pattern and the excitation coefficients of it are illustrated in Figure 3.10.

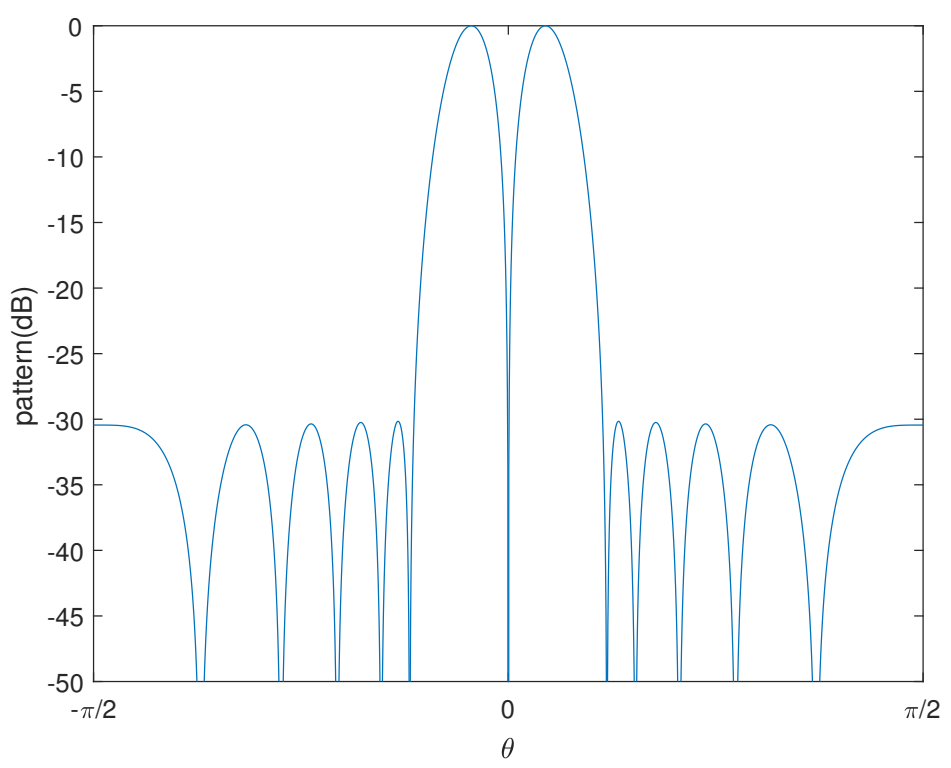

(a) Beam pattern 


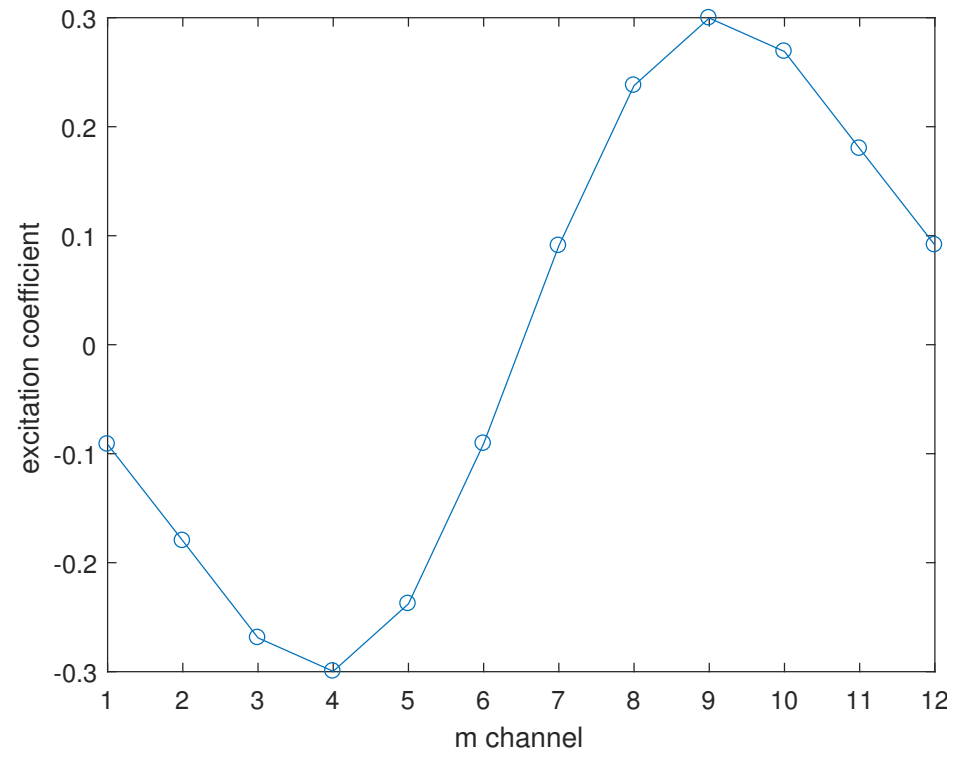

(b) Excitation Coefficients

Figure 3.10: Approximation to Zolotarev sythesis of sum pattern of 12 elements, $a=0.65$

Thus we generate a taper of difference beam through

$$
w_{\text {Zolotarev }}=\left[\begin{array}{llll}
f(1) & f(2) & \cdots & f(M)
\end{array}\right] \odot w_{\text {Chebyshev }} .
$$

Then the sum and difference pattern of using Chebyshev and Zolotarev synthesis are defined as

$$
\begin{gathered}
P_{\Sigma}(\theta)=\mathbf{w}_{\Sigma}^{H}\left(w_{\text {Chebshev }} \odot \mathbf{a}(\theta)\right) \\
P_{\Delta}(\theta)=\mathbf{w}_{\Delta}^{H}\left(w_{\text {Zolotarev }} \odot \mathbf{a}(\theta)\right),
\end{gathered}
$$

where $\mathbf{w}_{\Sigma}=\left(w_{\text {Chebshev }} \odot \mathbf{a}\left(\theta_{0}\right)\right)$, and $\mathbf{w}_{\Delta}=\left(w_{\text {Zolotarev }} \odot \mathbf{a}\left(\theta_{0}\right)\right)$. Similar to phase comparison method, the error voltage after synthesis is also the imaginary part of the monopulse ratio.

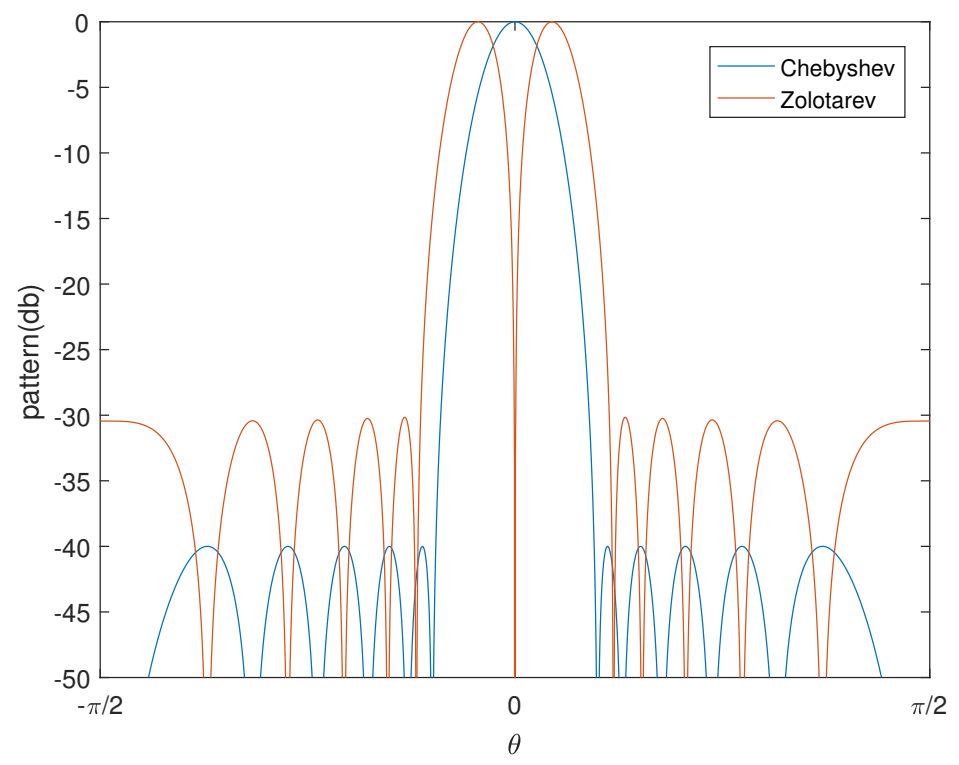

(a) Beam pattern 


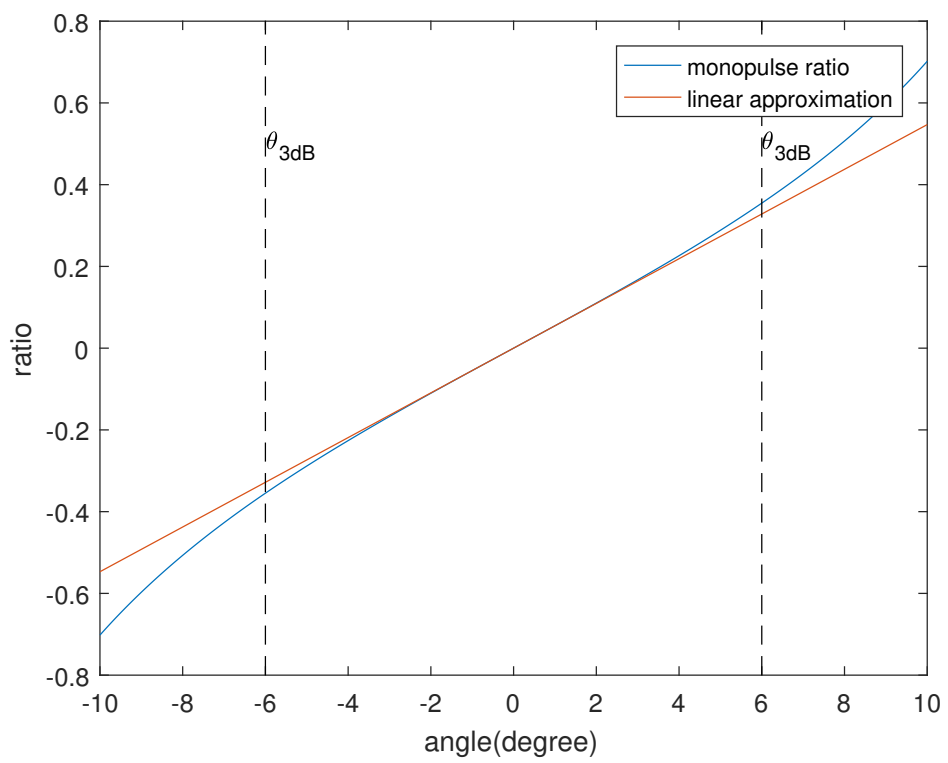

(b) MRC using Chebyshev and Zolotarev synthesis

Figure 3.11: Sum and difference pattern, monopulse ratio using Chebyshev and Zolotarev synthesis

The proposed synthesis method of generating sum and difference beam pattern has the following beneficial property than conventional amplitude and phase comparison method. Firstly the Chebyshev sum beam has controlled level of sidelobe, as well as maintaining the minimum beamwidth. And the Zolotarev weighting also yields the equal sidelobe level for difference pattern in the same sense as the Chebyshev weighting. Secondly, the Zolotarev difference beam generates a deep nulling at the looking direction, which makes it more sensitive to the angle bias of the targets and thus results in nice performance in estimation accuracy. However the proposed synthesis of beam will increase the width of the main beam width. As is shown in Figure 3.11 (b), compared with Figure 3.6 (a) and Figure 3.8 (b), we have a wider linear region because the Chebyshev weighting will increase the mainlobe beamwidth compared with the conventional DBF. This leads to a less sharp slope of MRC, and will slightly decrease the accuracy of tracking the target using error voltage. In Chapter 5, we will demonstrate that such a decline of accuracy is quite small to be neglected, while on the other hand, our proposed method has a better linear approximation within the $-3 \mathrm{~dB}$ beamwidth.

\begin{tabular}{cccc}
\hline & Amplitude comparison & Phase comparison & The proposed synthesis \\
\hline Approximation RMSE & $0.049^{\circ}$ & $0.017^{\circ}$ & $0.010^{\circ}$ \\
\hline
\end{tabular}




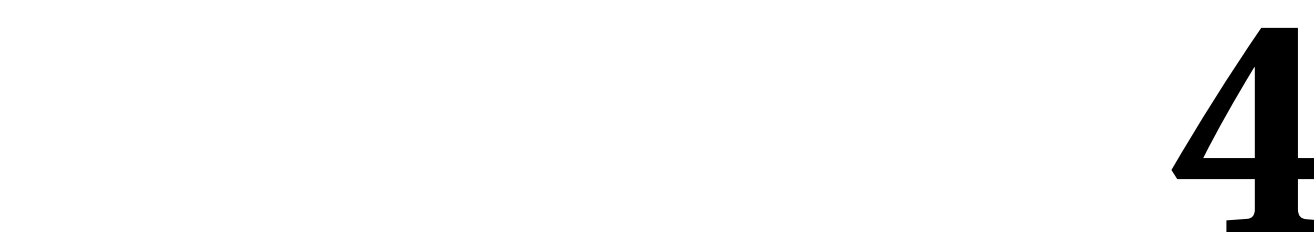

Clutter Suppression Using STAP AdAPTIVE MiMO-MONOPULSE

In radar detection, the interference due to clutter and jamming will be quite variable as a function of range, angle, and Doppler cells in the vicinity of a target. Clutter is defined as unwanted echoes reflected from the environment such as ground, sea and atmospheric turbulence. Jamming signals can be often related to a false target from a specific direction spread over all Doppler frequency, which becomes significant spatial interference.

If the target reflection is not strong enough, (for example to detect a pedestrian), the most severe case exists when the dominant source is clutter and especially if its amplitude exceeds that of the target signal. In this case, spectral signal processing is often employed (moving target indication (MTI) or pulse-Doppler processing) to reduce the clutter level below that of the target signal. In cases where the dominant interference is jamming and its level exceeds that of the target, often DOA processing (e.g., sidelobe cancellation, adaptive beamforming) may be used. Systems suffering significant clutter and jamming interference may use a combination of both, called space-time adaptive processing (STAP).

In application of automotive radar, it is necessary to suppress the reflected undesirable clutter from such objects as the ground surface (especially hills), guard rails, and buildings, in order to detect moving targets. Besides moving target detection, suppression of clutter is also significant for DOA estimation, especially in an automotive radar application, since the clutter comes from all direction, and the platform of radar is a vehicle which is always not stationary, thus the spectrum of clutter will result in a wide spread of Doppler frequency. Since we attempt to apply the proposed MIMO monopulse algorithm on pedestrian tracking, the effect of strong clutter could be severe since the RCS of a pedestrian target is sometimes very low, even weaker than the reflection of clutter. False and miss detection is expected to be avoided through the combination of STAP 
with MIMO-monopulse.

Jamming suppression is a common mission in airborne radar and military application. For automotive radar, the jammer can be received if there exist other radars in sight which keeps transmitting signals. Since the $79 \mathrm{GHz}$ band is isolated to other radio application, jammer might not be a strong interference on the automotive radar. But we will still consider jammer as spatial interference to introduce the concept of adaptive beamforming. Although in a realistic scenario the model of the jamming signal is more complex, in this thesis we just consider a simple case that a Continuous Waveform modulated signal from a fixed direction over the bandwidth of FMCW signals is received. Such interference has the spectrum through the whole range profile.

\subsection{SPACE-TIME SignALS}

STAP is an adaptive filtering technique using spatial and temporal degrees of freedom. It is essentially an extension of spatial beamforming to two-dimensional angle-Doppler beamforming. In STAP processing, firstly we need to define space-time signals. Consider the deramped FMCW signal

$$
s(t)=\exp \left\{j 2 \pi\left(\frac{2 f_{c} \cdot R}{c}+\left(\frac{2 f_{c} \cdot v}{c}+\frac{2 k \cdot R}{c}\right) t\right)\right\} .
$$

The $l$ th ramp within the repetition interval $T_{P R I}$ is expressed as

$$
\begin{aligned}
s_{2 D}(t, l) & =\exp \left\{j 2 \pi\left(\left(\frac{2 f_{c}\left(R+v T_{P R I} l\right)}{c}\right)+\left(\frac{2 f_{c} v}{c}+\frac{2 k\left(R+v T_{P R I} l\right)}{c}\right) \cdot t\right)\right\} \cdot \operatorname{rect}\left(\frac{t-l T_{P R I}}{T_{P R I}}\right) \\
& \approx \exp \left\{j 2 \pi\left(\left(\frac{2 f_{c}\left(R+v T_{P R I} l\right)}{c}\right)+\left(\frac{2 f_{c} v}{c}+\frac{2 k R}{c}\right) \cdot t\right)\right\} \cdot \operatorname{rect}\left(\frac{t-l T_{P R I}}{T_{P R I}}\right) .
\end{aligned}
$$

We assume the velocity is constant with in $T_{P R I}$. Suppose the sampling frequency is $F_{s}$, firstly we take one dimension fast fourier transform on fast-time samples to obtain range profile,

$$
S(f, l)=\exp \left(j 2 \pi \frac{2 f_{c} R}{c}\right) \sum_{n=0}^{N-1} \exp \left(j 2 \pi \frac{2 v T_{P R I} l f_{c}}{c}\right) \cdot \exp \left(\left(\frac{2 f_{c} v}{c}+\frac{2 k R}{c}\right) \frac{n}{F_{s}}\right) \cdot \exp \left(-j 2 \pi \frac{n \cdot f}{N_{F F T}}\right),
$$

where $N_{F F T}$ is the length of FFT. It can be seen that only the term of $n$ will be transformed into function of $f$, which we express as $S_{\text {range }}(f)$. Thus it can be written as:

$$
S(f, l)=\exp \left(j 2 \pi \frac{2 f_{c} R}{c}\right) \cdot \exp \left(j 2 \pi \frac{2 v T_{P R I} l f_{c}}{c}\right) \cdot S_{\text {range }}(f) .
$$

Consider the direction of arrival, the three dimensional FMCW signal after FFT on range is given by:

$$
\begin{aligned}
X\left(\theta_{0}, v, f\right) & =A\left(\theta_{0}\right) S(f, l) \\
& =\alpha \cdot \exp \left(j 2 \pi(M-1) \frac{d \sin \left(\theta_{0}\right)}{\lambda}\right) \cdot \exp \left(j 2 \pi \frac{2 v T_{P R I} l f_{c}}{c}\right) \cdot S_{\text {range }}(f),
\end{aligned}
$$

which is a $3 \mathrm{D}$ data cube with size of $M \times L \times N$, where $M$ is the number of sensor, $L$ is the number of slow time snapshots and $N$ is the length of fast time snapshots. $\alpha=\exp \left(j 2 \pi \frac{2 f_{c} R}{c}\right)$ is just a scalar which can be ignored for simplification. In each range cell, it is $2 \mathrm{D}$ vector $X\left(\theta_{0}, v\right)$. 


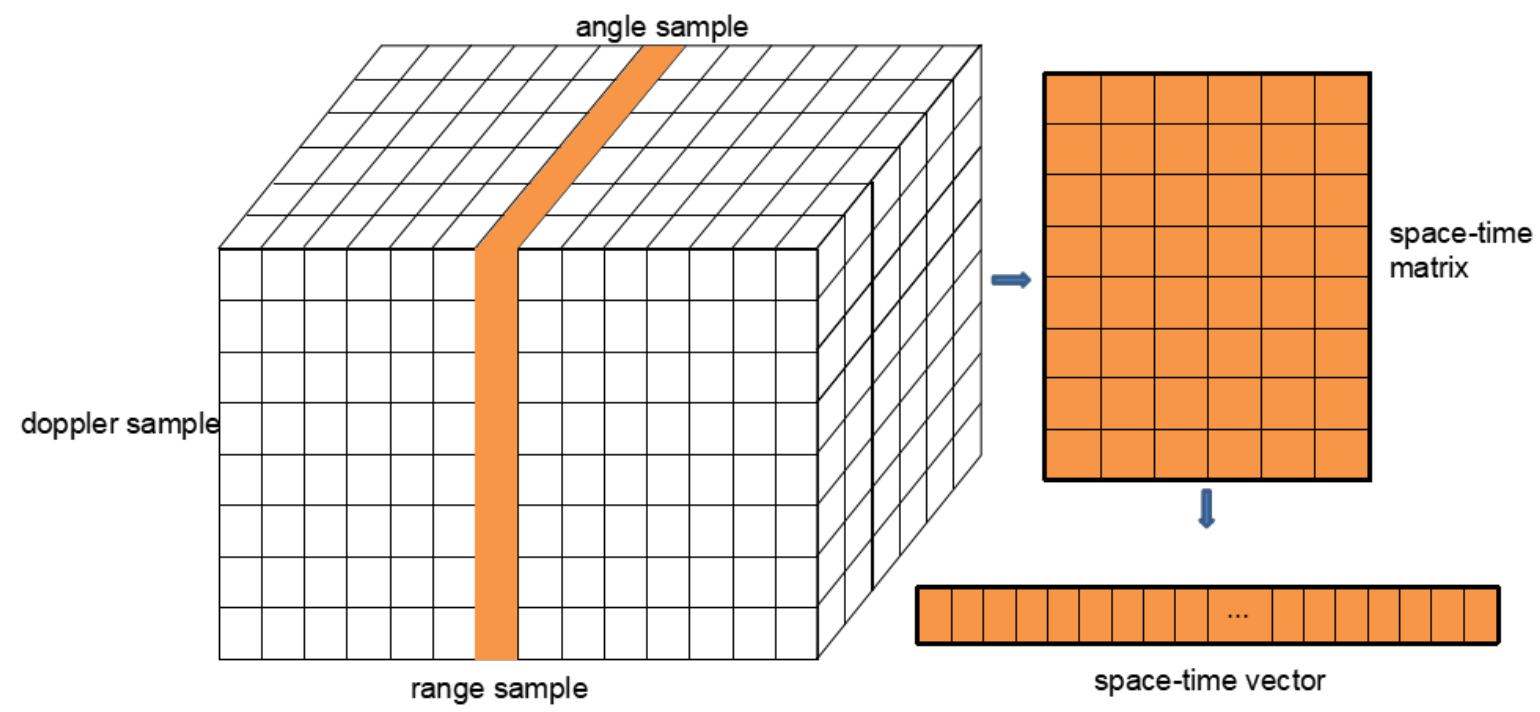

Figure 4.1: Space time signals in 3D radar data cube, which is obtained by extracting spatial and slow-time snapshots in one range cell and reshape the matrix into vector

Similar to the spatial steering vector definition given by Chapter 3 equation..., the Doppler frequency can also form temporal steering vector,

$$
\mathbf{b}\left(f_{d}\right)=\left[\begin{array}{lllll}
1 & e^{j 2 \pi f_{d} T_{P R I}} & e^{j 2 \pi 2 f_{d} T_{P R I}} & \cdots & e^{j 2 \pi(L-1) f_{d} T_{P R I}}
\end{array}\right]
$$

where $f_{d}$ is the Doppler frequency which is defined as $\frac{2 v f_{c}}{c}=\frac{2 v}{\lambda}, v$ is the line-of-sight velocity of the target, and $L$ is the number of pulses. Similar to the MIMO model that the signal is expressed as the product of steering Matrix and chirp signal, we can also express the space-time signal using spatial and temporal steering vector. Define the spatial-temporal steering vector as:

$$
\begin{aligned}
\mathbf{a}_{s-t}\left(\theta, f_{d}\right) & =\mathbf{b}\left(f_{d}\right) \otimes \mathbf{a}(\theta) \\
& =\left[\begin{array}{lllll}
1 \cdot \mathbf{a}(\theta) & e^{j 2 \pi f_{d} T_{P R I}} \cdot \mathbf{a}(\theta) & e^{j 2 \pi 2 f_{d} T_{P R I}} \cdot \mathbf{a}(\theta) & \cdots & e^{j 2 \pi(L-1) f_{d} T_{P R I}} \cdot \mathbf{a}(\theta)
\end{array}\right] .
\end{aligned}
$$

Through spatial-temporal steering vector, equation 4.5 can be reshaped into a two dimensional signal.

$$
X_{s t}\left(\theta_{0}, \nu, f\right)=\mathbf{a}\left(\theta_{0}\right) \otimes \mathbf{b}\left(f_{d}\right) \cdot S_{\text {range }}(f)=\mathbf{a}_{s-t}\left(\theta_{0}, f_{d}\right) \cdot S_{\text {range }}(f) .
$$

The dimension of $X_{s t}$ is $M L \times N$, which will be used for STAP. Analog to DBF discussed in Section 3.1, the non-adaptive space-time weighting vector can be defined as

$$
\mathbf{w}_{s-t}=\mathbf{a}_{s-t}\left(\theta_{0}, f_{d}\right) .
$$

In MIMO radar, both direction-of-arrival (DOA) information and direction of departure (DOD) information are available. This allows for discrimination between direct path backscatter and multipath backscatter. Direct-path backscatter will exhibit a DOA that is identical to the DOD, while multipath backscatter will exhibit a DOA that is different than the DOD. If MIMO radar is added to the STAP formulation, there will be three adaptive dimensions (DOA, DOD, and Doppler shift) as opposed to two with traditional STAP (DOA and 
Doppler shift). MIMO STAP should be able to suppress multipath clutter without suppressing the target, even if they share the same DOA and Doppler shift, by taking advantage of the DOD dimension[52, 53]

Consider the steering vector of transmitter and receiver array separately, redefine the spatial-temporal steering vector as

$$
\mathbf{a}_{s-t}\left(\theta_{R}, f_{d}, \theta_{T}\right)=\mathbf{a}_{T}\left(\theta_{T}\right) \otimes \mathbf{b}\left(f_{d}\right) \otimes \mathbf{a}_{R}\left(\theta_{R}\right),
$$

where $\theta_{T}, \theta_{R}$ denote the direction of depature (DOD) and direction of arrival (DOA).

\subsection{SPACE-Time Adaptive Monopulse}

\subsubsection{AdAPtive Digital BeAmFormer}

A disadvantage of the MIMO-monopulse estimator discussed in Chapter 3 is that it cannot provide suppression of jamming, clutter or other forms of interference. Adaptive monopulse tries to utilize adaptive beamforming weighting vector to generate adaptive sum and difference beam. We first consider spatial adaptive monopulse, which is an effective manner to counter the problem of sidelobe jamming. Review the expression of digital beamforming

$$
y=\mathbf{w}^{H} \mathbf{x} .
$$

In this case, we consider the data matrix is composed of both signals from targets and jammers. The fundamental problem in adaptive beamforming is to find a weight vector $\mathbf{w}$, based only on observing the total array data vector $\mathbf{x}$, that minimizes the jammer term without degrading the signal term. Suppose we have $J$ clutters with steering vector $\mathbf{v}\left(\theta_{j}\right)$. Then the weighting vector should be ideally chosen that

$$
\mathbf{w}^{H} \mathbf{v}\left(\theta_{j}\right)=0, \quad j=1,2, \cdots, J .
$$

Normally the solutions of above equation cannot guarantee that the interference is suppressed while preserving the desired signals, which is the goal of adaptive beamforming. Adaptive algorithms determine the best solution for the weights by performing an optimization with respect to selected statistical performance criteria.

Most adaptive beamforming algorithms are based on optimizing the weight vector with respect the power of various signals after beamforming, such as minimizing the mean square error (MSE) signal or maximizing the signal power relative to the jammer plus noise power (SINR). If the optimization criterion for the adaptive filter is to minimize MSE, then the resulting solution for the adaptive weights is known as the Wiener filter

$$
\mathbf{w}_{\text {opt }}=\mathbf{R}_{x}{ }^{-1} \mathbf{a}\left(\theta_{0}\right)
$$

The Wiener filter and the maximum SINR solutions both maximize the SINR for the beam steered to the desired direction, but otherwise, they don't constrain the antenna pattern response, which can lead to highly distorted antenna patterns. Normally this can be solved by using constrained optimization to compute the weights. The linear constrained minimum variance (LCMV) [54] solution for the weights minimizes the out- 
put power of the beamformer

$$
\begin{aligned}
& \min \quad \mathbf{w}^{H} \mathbf{R}_{x} \mathbf{w} \\
& \text { s.t. } \quad \mathbf{C}^{H} \mathbf{w}=\mathbf{g} .
\end{aligned}
$$

Where $\mathbf{R}_{x}$ is the covariance matrix which is defined as $\mathbf{R}_{x}=E\left[\mathbf{x x}^{H}\right]$. In jammer suppression adaptive beamforming, the covariance matrix is consists of signal covariance, jammer covariance and white noise covariance, which are assumed to be mutually uncorrelated and expressed as:

$$
\mathbf{R}_{x}=\mathbf{R}_{s}+\mathbf{R}_{j}+\mathbf{R}_{n}
$$

Using the Lagrange multipliers method, the solution of this constrained optimization problem is:

$$
\mathbf{w}_{L C M V}=\mathbf{R}_{x}{ }^{-1} \mathbf{C}\left(\mathbf{C}^{H} \mathbf{R}_{x}{ }^{-1} \mathbf{C}\right)^{-1} \mathbf{g} .
$$

Assume $\mathbf{C}$ is equal to the steering vector $\mathbf{a}\left(\theta_{0}\right)$ and $\mathbf{g}$ is equal to 1 for unity gain in that direction, which in this case is called the minimum variance distortionless response (MVDR) beamformer

$$
\mathbf{w}_{M V D R}=\frac{\mathbf{R}_{x}{ }^{-1} \mathbf{a}\left(\theta_{0}\right)}{\mathbf{a}\left(\theta_{0}\right)^{H} \mathbf{R}_{x}{ }^{-1} \mathbf{a}\left(\theta_{0}\right)} .
$$

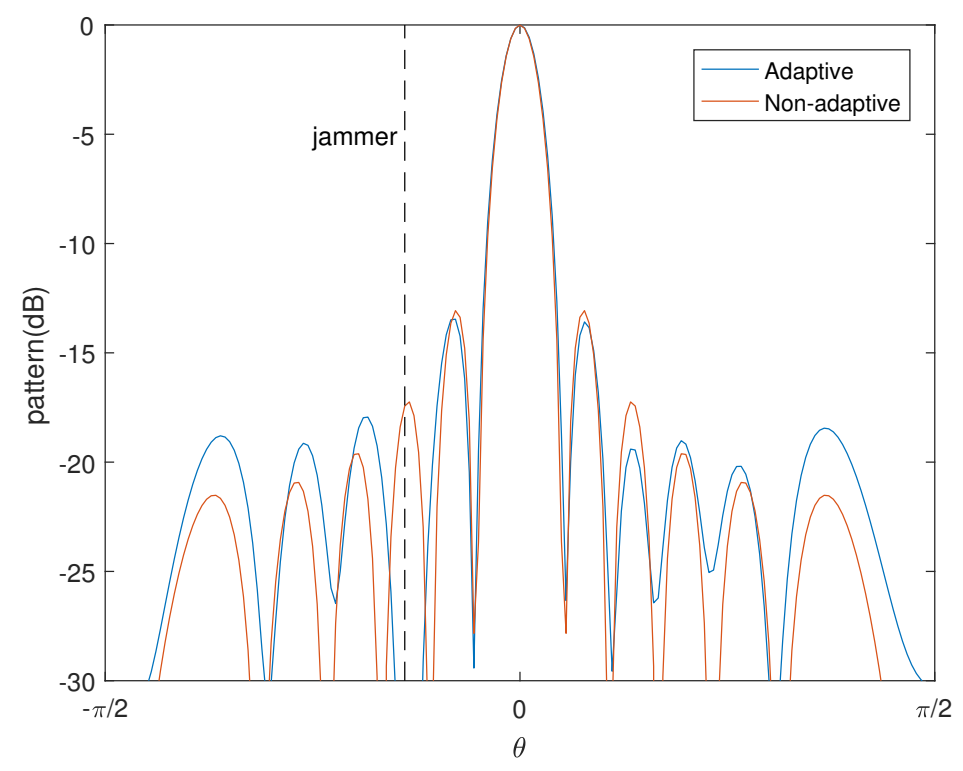

Figure 4.2: Adaptive beamforming. A jammer is from $-25^{\circ}$

The MVDR adaptive weighting is used if the target-free covariance matrix is unavailable, and only the target-plus-interference-plus-noise covariance matrix is available (which would be the case if the target is present in the training data, for example, if you train over slow-time pulses), The concept of adaptive beamforming can be extended to spatial-temporal domain to process space-time signals in order to suppress clutter which exhibits correlation in the spatial and slow-time dimensions and generally appears white in fast time. 


\subsubsection{SPACE-Time AdAPtive Sum and DifFEREnCE BEAMFormer}

Given the expression of space-time signals in equation 4.8, in each range cell we have a column vector $\mathbf{x}_{s-t}$, The space-time adaptive sum and difference beamformer can be yielded through MVDR solution. The adaptive sum beamformer can be solved by:

$$
\begin{array}{ll}
\min _{\mathbf{W}_{\Sigma}} & \mathbf{W}_{\Sigma}^{H} \mathbf{R}_{x_{s-t}} \mathbf{W}_{\Sigma} \\
\text { s.t. } & \mathbf{a}_{\mathrm{s}-\mathrm{t}}\left(\theta_{0}, f_{d}\right)^{H} \mathbf{W}_{\Sigma}=1 .
\end{array}
$$

As for difference beamformer, since in chapter 3 we generate difference beam pattern through Zolotarev synthesis, if we express the Zolotarev taper as $w_{\text {Zolotarev }}$, the difference beamformer is obtained by $\mathbf{W}_{\Delta}=$ $w_{\text {Zolotarev }} \odot \mathbf{W}_{\Sigma}$. Thus it can be solved by:

$$
\begin{array}{ll}
\min _{\mathbf{W}_{\Delta}} & \mathbf{W}_{\Delta}^{H} \mathbf{R}_{x_{s-t}} \mathbf{W}_{\Delta} \\
\text { s.t. } & \left(w_{\text {Zolotarev }} \odot \mathbf{a}_{\text {s-t }}\left(\theta_{0}, f_{d}\right)\right)^{H} \mathbf{W}_{\Sigma}=1 .
\end{array}
$$

The covariance matrix $\mathbf{R}_{x_{s-t}}$ is composed of the covariance of the target, direct path clutter, multi-path clutter and white noise:

$$
\mathbf{R}_{x_{s-t}}=\mathbf{R}_{s}+\mathbf{R}_{c d}+\mathbf{R}_{c m}+\mathbf{R}_{n}
$$

The target's covariance matrix with angle of arrival $\theta_{R}$ (for target $\theta_{R}=\theta_{T}$ ), with Doppler frequency $f_{d}$, and power $\sigma_{s}^{2}$.

$$
\mathbf{R}_{s}=\sigma_{s}^{2} \mathbf{a}\left(\theta_{R}, f_{d}, \theta_{T}\right) \mathbf{a}\left(\theta_{R}, f_{d}, \theta_{T}\right)^{H}
$$

Similarly, the covariance of direct path clutter is given as

$$
\mathbf{R}_{c d}=\int_{\theta \in \Theta_{c d}} \sigma_{c d}(\theta)^{2} \mathbf{a}\left(\theta, f_{c d}(\theta), \theta\right) \mathbf{a}\left(\theta, f_{c d}(\theta), \theta\right)^{H} \mathrm{~d} \theta,
$$

the power of the clutter patch at angle $\theta$ is expressed as $\sigma_{c d^{2}}$, and the Doppler frequency of the clutter patch is $f_{c d}$. The setting $\Theta_{c d}$ includes all directions that clutter exists. The DOD and DOA are equal in the case of direct path clutter.

The multipath clutter covariance matrix is written as:

$$
\mathbf{R}_{c m}=\int_{s \in \Gamma_{c m}} \sigma_{m d}(s)^{2} \mathbf{a}\left(\theta_{R}, f_{c m}(s), \theta_{T}\right) \mathbf{a}\left(\theta_{R}, f_{c m}(s), \theta_{T}\right)^{H} \mathrm{~d} s
$$

where $\sigma_{c m}{ }^{2}$ represents the power of the clutter patch at receiver azimuth $\theta_{R}$ and transmit azimuth $\theta_{T}$, and the Doppler shift of the clutter patch is $f_{c m}$. The auxiliary parameter $s$ is used to define the case of two different types of multi-path clutter [53]: The first basic type occurs when the transmitted signal scatters first from the strong scatterer to a clutter locus, then to the receiver. The set $\Gamma_{c m}$ contains all the combinations of transmit-receive angle. Finally, the noise covariance matrix is expressed as

$$
\mathbf{R}_{n}=\sigma_{n}^{2} \mathbf{I}
$$

The covariance matrix $\mathbf{R}_{x}$ is not prior known in practice and thus needs to be estimated as follows

$$
\hat{\mathbf{R}}_{x_{s-t}}=\frac{1}{K} \sum_{k=1}^{K} \mathbf{x}_{s-t}(k) \mathbf{x}_{s-t}(k)^{H},
$$


where $\mathbf{x}_{s-t}(k)$ denotes the space-time samples in $k$ th range cell, which normally doesn't include the range cell of interest and some guarding cells around. In [55] it indicates that for the SINR loss to be no more than $3 \mathrm{~dB}$ in the mean requires that the number of data samples be roughly twice the number of the spatial-temporal channels $(K=2 M L)$. 



\section{Implementation AND PerformanCe}

\section{EVALUATION}

In this chapter, the implementation of MIMO-monopulse DOA estimation algorithm based on MATLAB simulation will be analyzed. The potential application of MIMO-monopulse on pedestrian tracking will be discussed. Furthermore, the algorithm is tested on real experimental data collected by an NXP Dolphin automotive radar.

\subsection{Implementation Based on Matlab Simulation}

\subsubsection{FMCW SIGNAL GENERATION}

FMCW Signal generation is realized based on MATLAB which built the signal model fixed with realistic data collected by the NXP Dolphin radar. The parameters of simulated FMCW chirp signal is shown in Table 5.1 


\begin{tabular}{llr}
\hline Description & Name & \\
\hline Center frequency & $F_{c}$ & $79 \mathrm{GHz}$ \\
Sweep bandwidth & $B$ & $1 \mathrm{GHz}$ \\
Chirp duration & $T_{c}$ & $36.66 \mu \mathrm{s}$ \\
Dwell time & $T_{\text {Dwell }}$ & $1 \mu \mathrm{s}$ \\
Settle Time & $T_{\text {Settle }}$ & $5 \mu \mathrm{s}$ \\
Reset time & $T_{\text {Reset }}$ & $5 \mu \mathrm{s}$ \\
Slope of ramp frequency & $\mu$ & $32.68 \mathrm{MHz} / \mu \mathrm{s}$ \\
Sampling frequency & $F_{s}$ & $40 \mathrm{MHz}$ \\
Number of samples in chirp & $N_{c}$ & 1464 \\
Number of samples for processing & $N$ & 1024 \\
\hline
\end{tabular}

Table 5.1: Parameters of simulated FMCW signal

The simulated model uses 12 spatial channels for DOA estimation (formed by 3 transmitters and 4 receivers MIMO array), 16 slow-time snapshots for Doppler processing and 1024 fast-time snapshots for range processing. Each transmitter emits FMCW signals (IF signal) modulated to center frequency (RF signal) sequentially to generate Time Division orthogonal waveforms. The received signals are generated by timedelayed transmitted signals. The delay is calculated using equation $2(R+v t) / c$. To generate reflected echo from targets, radar range equation is calculated to get the received power and signal to noise ratio (SNR) of the target, which is defined as

$$
\begin{gathered}
P_{r}=\frac{P_{t} G_{t} G_{r} \lambda^{2} \sigma}{(4 \pi)^{3} R^{4} L} \\
S N R=\frac{P_{r}}{N}=\frac{P_{r} \tau}{k T_{s}},
\end{gathered}
$$

where $P_{r}, P_{t}$ are the received and transmitted power, $G_{t}, G_{r}$ are transmitter and receiver gain, $\sigma$ is target's nonfluctuating radar cross section in square meters, $R$ is the distance from radar to the target, and $L$ is the general loss factor. To calculate SNR, $k$ is the Boltzmann constant and $T_{S}$ is the system temperature.

Then the complex conjugate of down-converted received signal (IF signal) is multiplied with transmitted signal to generate deramped signal for processing. After deramping, the bandwidth of the signal is reduced considerably which allows lower sampling frequency.

\subsubsection{Processing Using 3D Fast Fourier Transform}

In FMCW signal generation part we obtain a 3D data cube with dimension $12 \times 16 \times 1024$. Apply 3D FFT on sensor, slow-time and fast-time, we can get obtain DOA, Doppler and range information.

The length of FFT of fast-time is $N_{F F T 1}=1024$, the mapping from frequency to range is given by $R=\frac{f_{r} \cdot c}{2 \mu}$, with the sampling frequency $F_{s}$ on fast-time domain. Thus the distance gate of range is from -91.8 meters to 91.6 meters, and the resolution is around 0.18 meters. Only the right side of the spectrum is required with positive range of targets. 
The length of FFT of slow-time is $\mathrm{NFFT}_{2}=32$, the relation between Doppler frequency and line-of-sight velocity to the radar is $v=\frac{f_{d} \cdot \lambda}{2}$. The sampling frequency of Doppler is defined as $F_{s, \text { Doppler }}=\frac{1}{T_{c}}$, where $T_{c}$ is the duration of a chirp. And the Doppler gate is from $-25.9 \mathrm{~m} / \mathrm{s}$ to $25.1 \mathrm{~m} / \mathrm{s}$.

For DOA, the length of FFT is $N_{F F T 3}=128$. Given the spatial frequency $F_{s \text {, angle }}=\frac{d}{\lambda} \sin (\theta)$, the DOA is expressed as $\sin ^{-1} \frac{F_{s, \text { angle }} \cdot \lambda}{d}$. Note that since the mapping from spatial frequency to angle is not a linear function, the scanning angle from $-90^{\circ}$ to $90^{\circ}$ is not uniformly distributed, which leads to the scanning step varying with direction (When the angle is large, the step is large), this may increase estimation error when the angle of the target is large.

The range-Doppler map and range-azimuth map using 3-dimensional Fast Fourier transform are shown in the Figure 5.1.

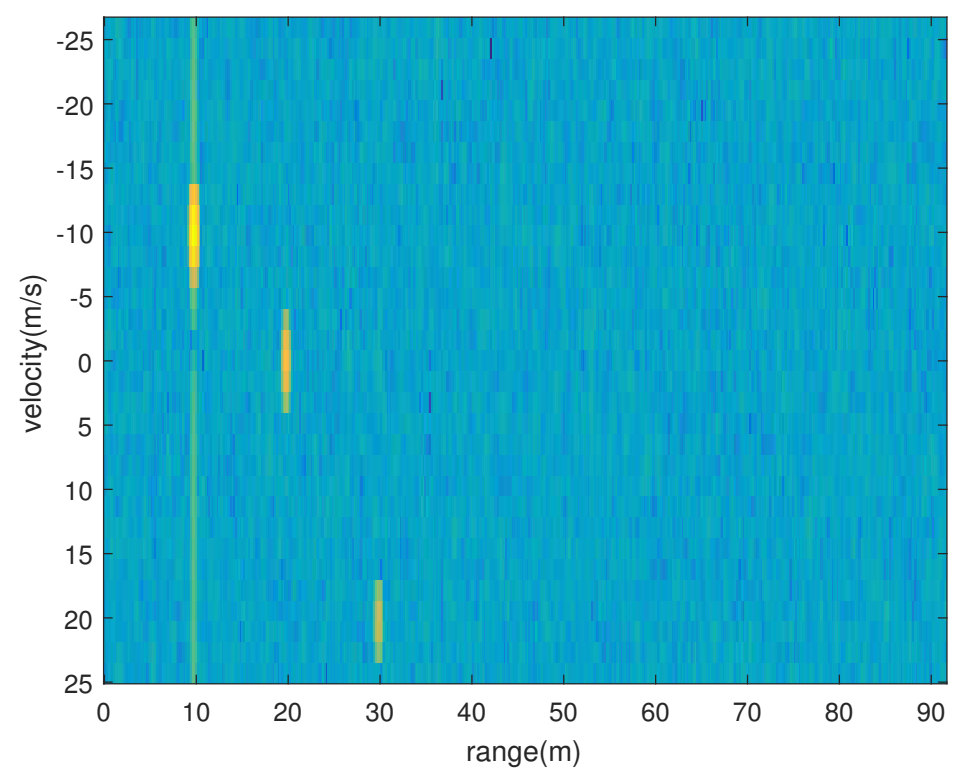

(a) Range Doppler map of simulation

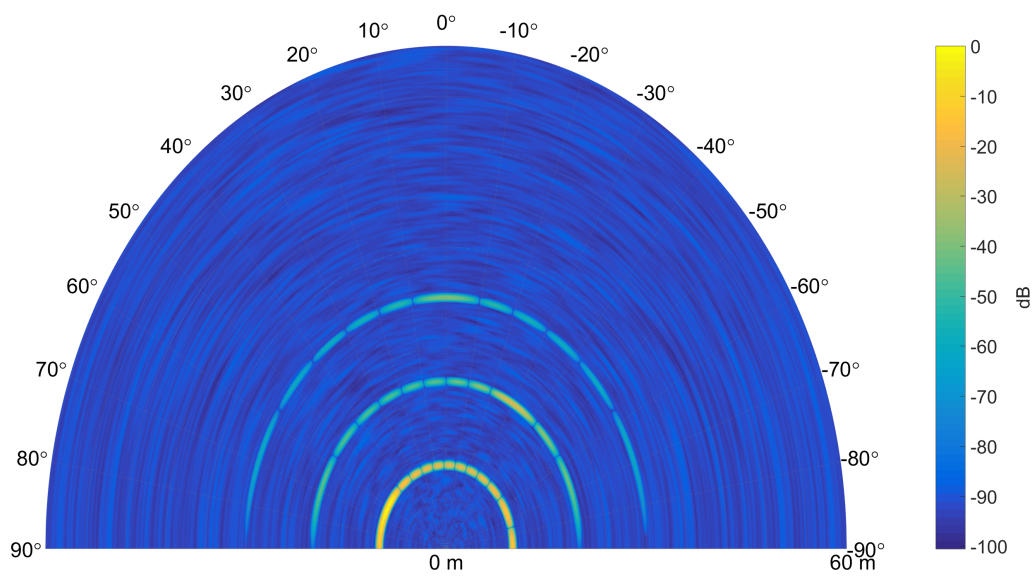

(b) Range-azimuth map of simulation

Figure 5.1: Simulation of processing FMCW signals using 3d FFT. Three targets with different range,velocity and DOA are visualized in range-Doppler and range-azimuth map 


\subsubsection{Digital BeAmForming}

After FFT processing on fast-time dimension, instead of taking FFT on spatial dimension, digital beamforming (DBF) is then applied to obtained data cube to obtain better performance of DOA estimation. Assume the chirp signal after FFT is $\mathbf{X}_{\mathrm{R}}$ To get the response of DBF pattern, beaming scanning is applied on the data of all range cell $n, n=1, \ldots, 1024$ from $-90^{\circ}$ to $90^{\circ}$ with step of 1 degree. Given the expression of DBF,

$$
\mathbf{y}(n)=\mathbf{w}^{H} \mathbf{X}_{\mathrm{R}}(n)
$$

Beam scanning means that we generate weighting vector on each step of scanning angle $\mathbf{w}_{i}=\mathbf{a}\left(\theta_{i}\right), i=$ $1, \cdots, 181$ to filter the data $X_{\mathrm{R}}$, then the response of DBF is thus written as:

$$
\mathbf{Y}=\left[\begin{array}{c}
\mathbf{w}_{1}^{H} \\
\mathbf{w}_{2}^{H} \\
\vdots \\
\mathbf{w}_{181}^{H}
\end{array}\right] \mathbf{X}_{\mathrm{R}}
$$

where $\mathrm{Y}$ is a $181 \times 1024$ matrix. The DOA of the target can be determined through searching the maximum of the output on angular axis.

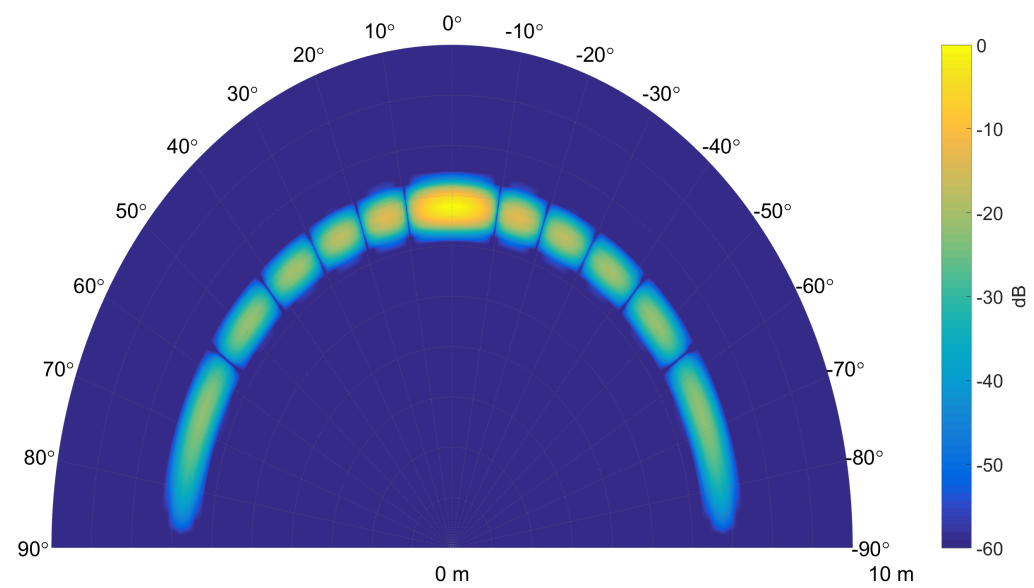

Figure 5.2: Range-azimuth map of DBF

\subsubsection{MIMO-MONOPULSE}

The implementation of MIMO-monopulse is quite different to standard or phased array monopulse. In these cases, the beam of the antenna is mechanically shifted to some direction, or the electromagnetic waves are steered by the phase shifter, to scan through a rotating beam step by step. The monopulse algorithm is always only applied within the main beam of radar, which is prior known and can be controlled to change. Once a target is detected within the main beam if the target is slightly biased to the looking direction, the error voltage is calculated through sum and difference beam, and the DOA is estimated by MRC.

In order to directly demonstrate the output of monopulse ratio, we want to visualize the result of MIMOmonopulse in a range-azimuth map, such as is shown in the Figure 5.2 using DBF. Since the monopulse ratio 
will be exactly 0 at the angle of the target, which we are interested in, if we take the opposite of absolute value of the monopulse ratio, there will exist a peak at the angle of the target. To make it similar with DBF response pattern, the result is then mapped to $\mathrm{dB}$ scale, and then a sharp peak is generated precisely at the direction of target.

However, there exist a problem of monopulse ratio ambiguity. In MIMO-monopulse, each element in the antenna array is omnidirectional, which radiates waves to wide azimuth and also receives reflections from all directions. The problem is that MIMO-monopulse doesn't know where is the main beam, and beam scanning is realized digitally to process the data. Thus the output will not only include the beam reflected from the target but also has ambiguity caused by sidelobe because these sidelobes can also generate zero crossing similar to monopulse ratio within the mainbeam, as is shown in Figure 5.3. Thus the biggest issue of MIMO-monopulse is to discard the ambiguity from sidelobe. One feasible solution is to detect the angle of the target using a fixed threshold on sum beam response, then keep the monopulse ratio within 3dB beamwidth around the detected target. In this thesis, we propose a simple solution via using a scalar to suppress zero point of monopulse ratio caused by sidelobe, and the scalar is carried out from sum beam response without detection. The procedure is:

1. Calculate the response of sum beam DBF;

2. Normalize the response to $[0,1]$, which becomes a vector of scalars with high value within the main beam and low value on outside;

3. Multiply the scalar with monopulse ratio in $\mathrm{dB}$ in the range of interest, which remains a high peak at main lobe, and weakens the peaks at sidelobes;

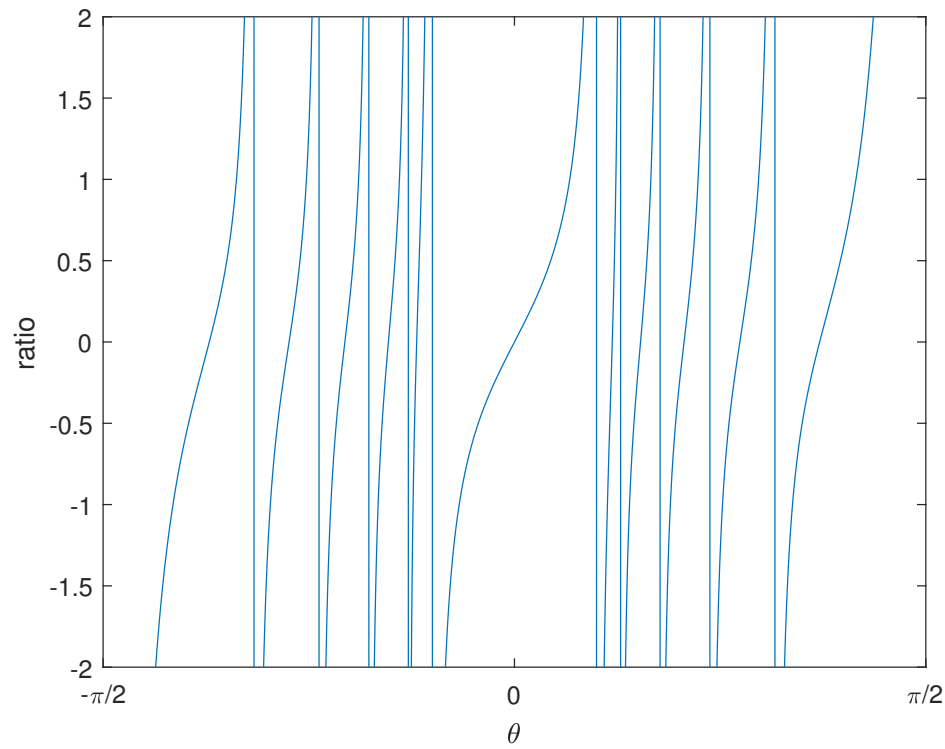

(a) monopulse ratio ambiguity 


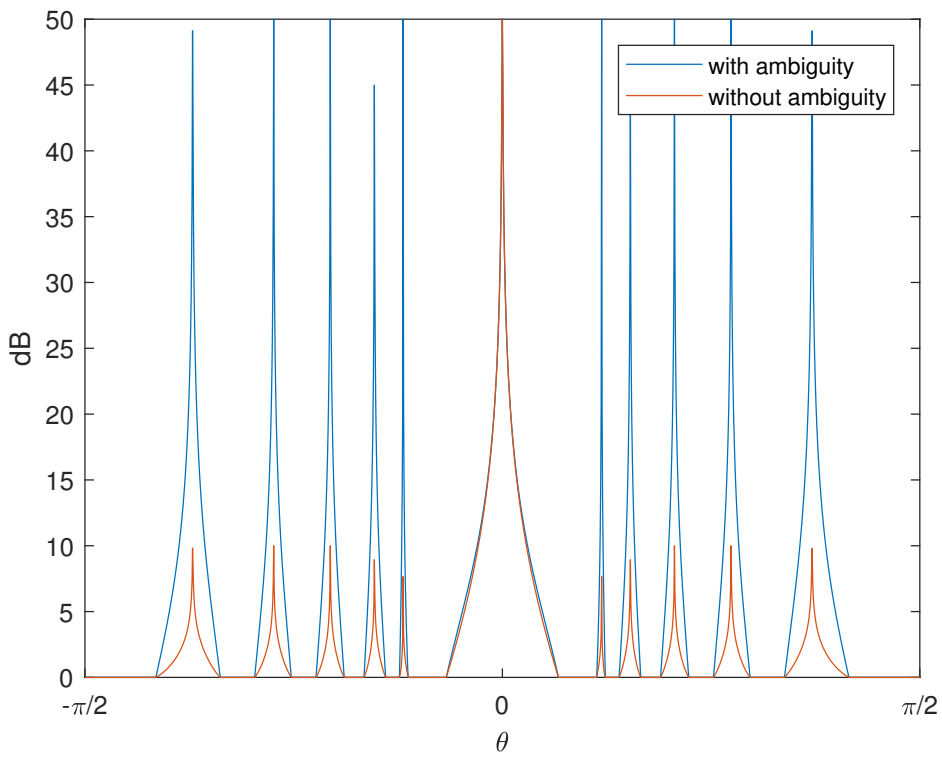

(b) monopulse ratio in $\mathrm{dB}$

Figure 5.3: Suppress the sidelobe using sum pattern

After this processing, the ambiguity of monopulse ratio is solved. Ambiguity caused by sidelobe ratio and the result after the proposed method is given in 5.3. As is shown in the figure, the monopulse ratio ambiguity is canceled which results in only high gain at the direction of the main lobe. Another advantage of such processing is that in the range-azimuth map of MIMO-monopulse, targets in different range cells with different RCS are possible to be visualized, because the spectrum of sum pattern includes both range information and reflection power of the targets.

The range-azimuth map of the sum and difference beams are shown below. Then monopulse is calculated and illustrated in $\mathrm{dB}$, which has a narrow peak at the direction of the target. From figure 5.4, it can be seen that MIMO monopulse demonstrates a much more accurate azimuth determination.

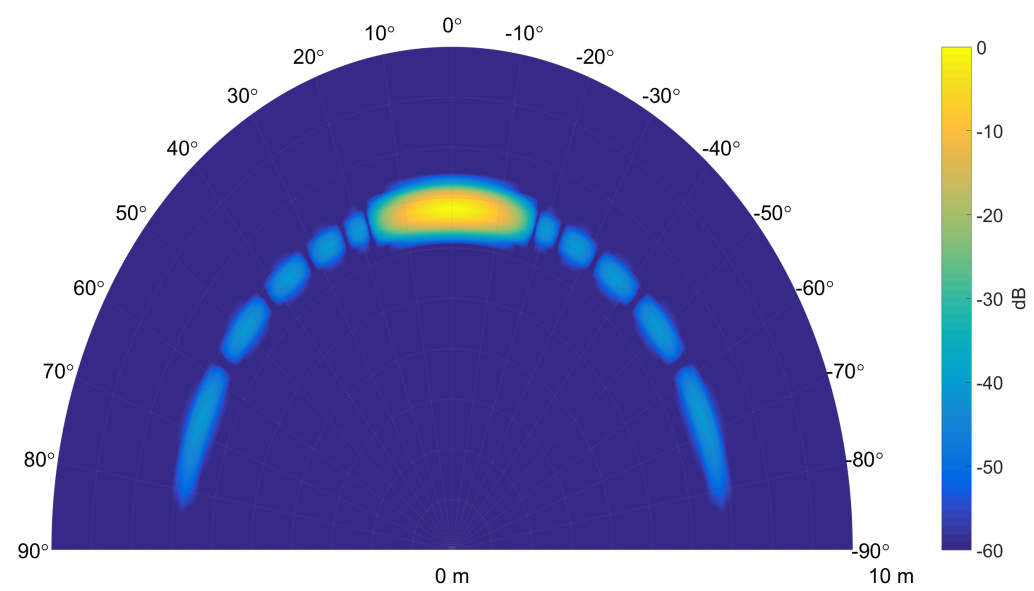

(a) Range-azimuth of sum pattern 


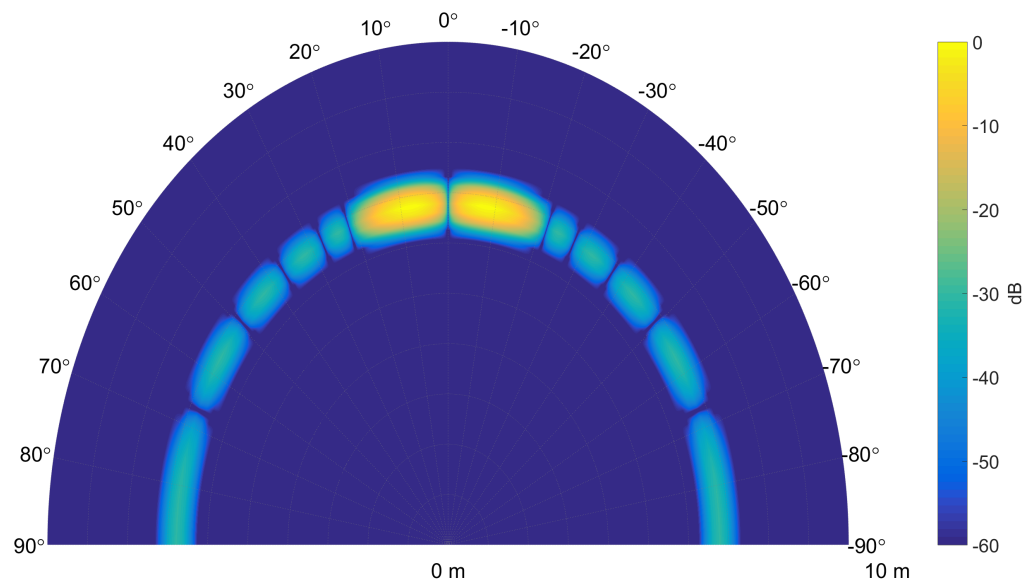

(b) Range-azimuth of difference pattern

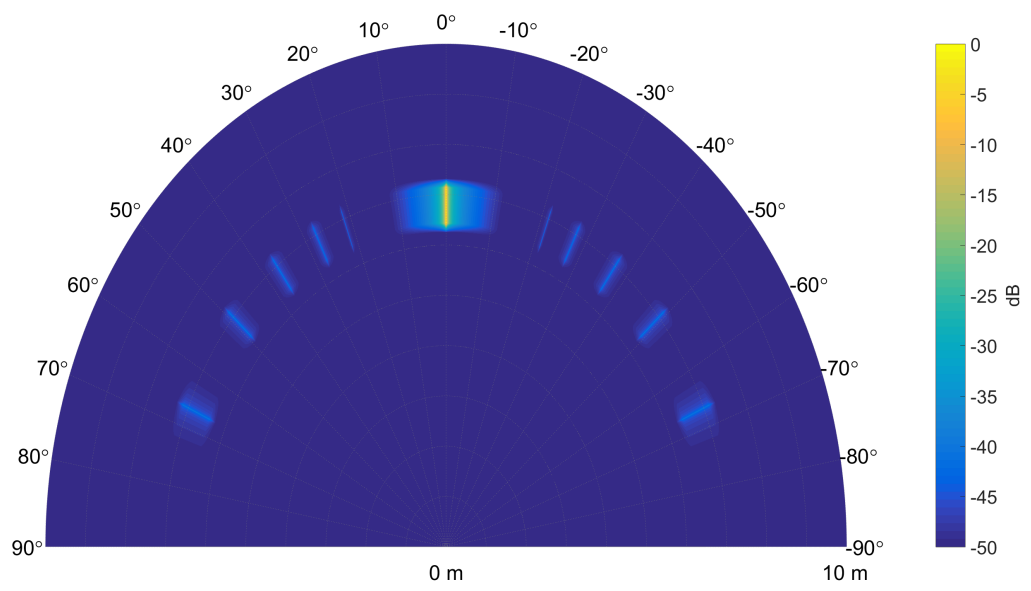

(c) Range-azimuth of monopulse ratio in $\mathrm{dB}$

Figure 5.4: Range azimuth after the proposed calibration, and test on different data sets

As is discussed in chapter 3, monopulse algorithm utilizes error voltage to estimate the angle of the target which is slightly deviate to the direction of the beam through MRC (the deviation is called error voltage). In this thesis, this property can be used for target tracking in the automotive radar application. Assume that DOA of one target in one pulse is estimated through monopulse ratio, which is determined by digitally beam scanning using sum and difference beam. The monopulse ratio will be exactly 0 at the direction of the target, and then it is visualized as a sharp peak in $\mathrm{dB}$ (as is illustrated in Figure 5.4 (c)). Then in next pulse, in order to estimate the DOA of target, it is not necessary to repeat beam scanning in all directions. The weighting vector of sum and difference beam obtained in the last scanning can still be used to filter the current pulse signals, however, the output of monopulse ratio will be not 0 if the target shifted in the angular axis. At present, the angle of the target can be estimated by linear approximation property within the local area around looking direction of the previous monopulse ratio and calculated through inverse mapping error voltage to MRC 3.12. 
Once this estimation is obtained, a new beam can directly be steered to this angle and then will be used for next pulse. Such procedure is repeated in several pulses to realize tracking of moving targets. Digital scanning using sum and difference is also needed to periodically update the weighting vectors after several pulses.

\subsubsection{COMPARISON WITH SUPER-RESOLUTION ALGORITHM}

In Chapter 2, we discussed the challenge of using a super-resolution algorithm for target localization in FMCW automotive radar application. One of the main advantages of MIMO-monopulse is that the DOA of targets can be estimated using only single pulse, which is called single snapshot in literature [56]. For superresolution algorithm such as MUSIC, the covariance matrix cannot be precisely estimated using a limited number of the measurement. The reason is that only the range bins of the target with peaks in the spectrum (normally one to three bins) are available for the direction of arrival estimation. The effect of the single snapshot of MUSIC is illustrated in Figure 5.5. In this simulation, spatial-smoothing (SS) of 5 overlapped subarrays is used to de-correlate the signal. Compared with the MUSIC, MIMO-monopulse has the capability of estimating the DOA of targets with the only single snapshot.

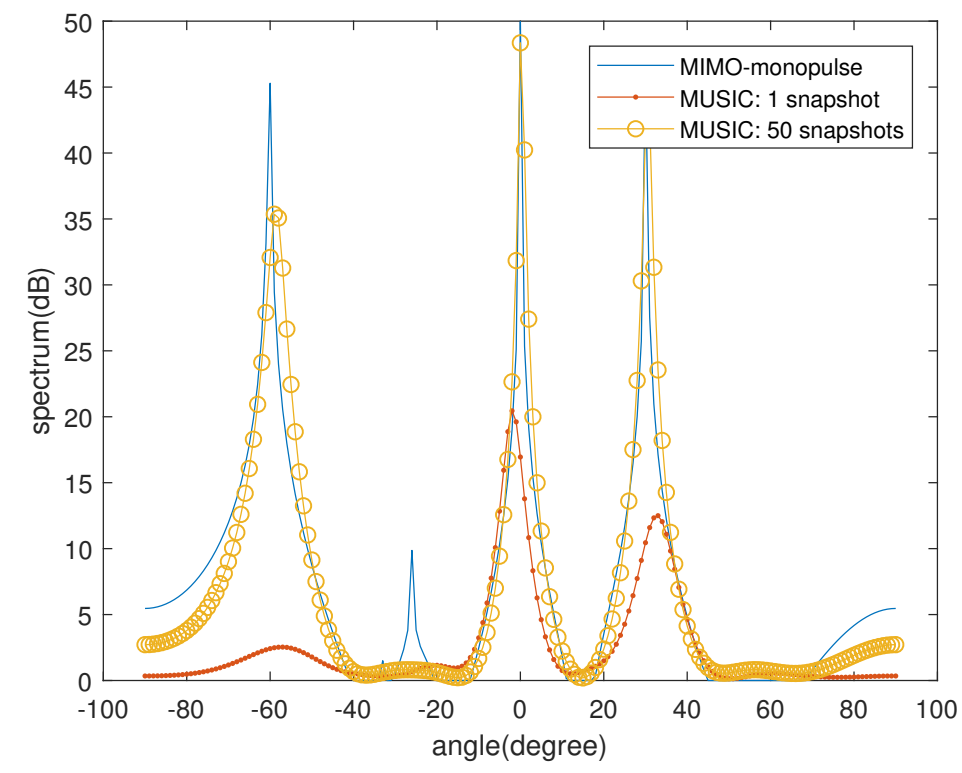

Figure 5.5: Single snapshot effect, SNR is $15 \mathrm{~dB}$

Another problem of super-resolution algorithms is the unknown number of targets. If the number of targets is not correctly prior estimated, the performance of MUSIC will dramatically deteriorate, which is shown in Figure 5.6. From this figure, if the dimension of subspace for targets is less than the real number, it will miss one target in angle estimation. For MIMO-monopulse, there is no such constraint. 


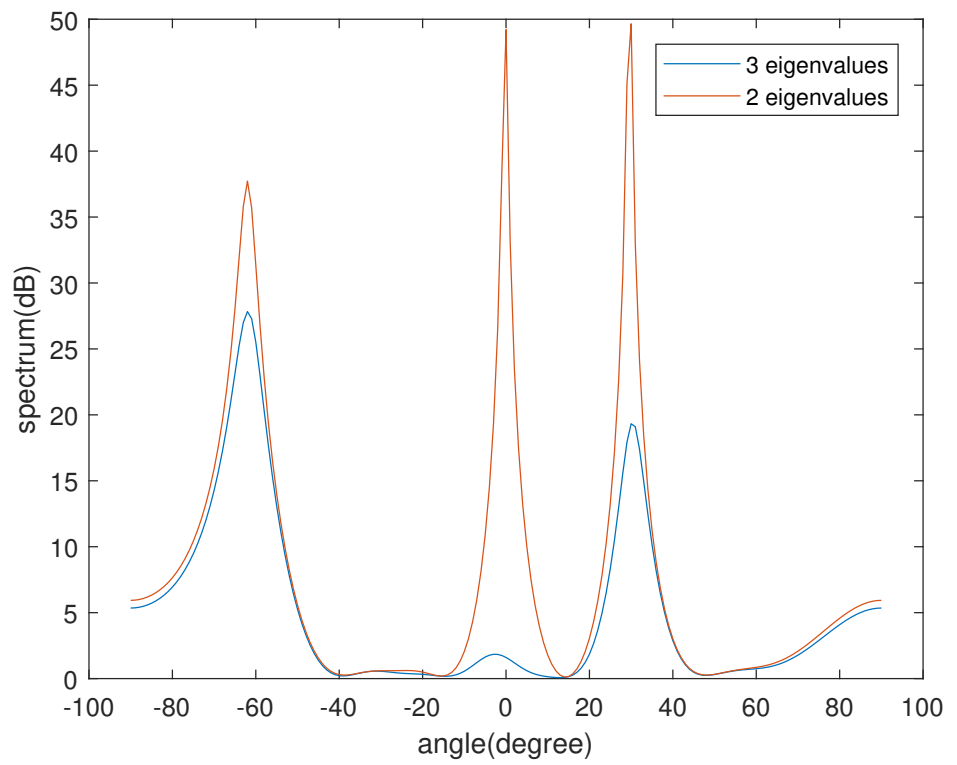

Figure 5.6: Wrong number of targets estimated

The state-of-the-art super-resolution algorithms utilize 2D joint estimation of range and azimuth. It solves many problems of MUSIC, however, the computation complexity is very high, which is not realistic for a real-time automotive radar system. Consider a signal $X$ with dimension $M \times N$, which represents the number of sensors and fast-time samples respectively. 2D MUSIC requires to reshape it into a $M N \times 1$ spatialtime vector, and normally needs $L$ vectors to estimate the covariance matrix. The eigenvalue decomposition should be applied to a matrix with $M N \times L$ dimension, which is computationally expensive for realization on a real automotive radar system.

\subsubsection{Performance Evaluation}

In chapter 3 we proposed the synthesis of sum and difference beam using Chebyshev and Zolotarev pattern. The advantage of using Chebyshev and Zolotarev pattern basically includes the less estimation error due to Chebyshev sidelobe reduction and much more sensitive monopulse ratio around the looking direction due to Zolotarev nulling. To demonstrate the benefits of the synthesis, we consider two simple cases of DOA estimation using MIMO-monopulse. In the first case, assume there are 3 targets separately distributed in one range cell. The estimation error of these 3 targets is calculated in 1000 Monte Carlo simulation, which is shown in the figure. From this result, it can be seen that if there are multiple targets in the same range cell, the sidelobe will affect the accuracy of estimation, thus the proposed method of synthesis presents better performance. 


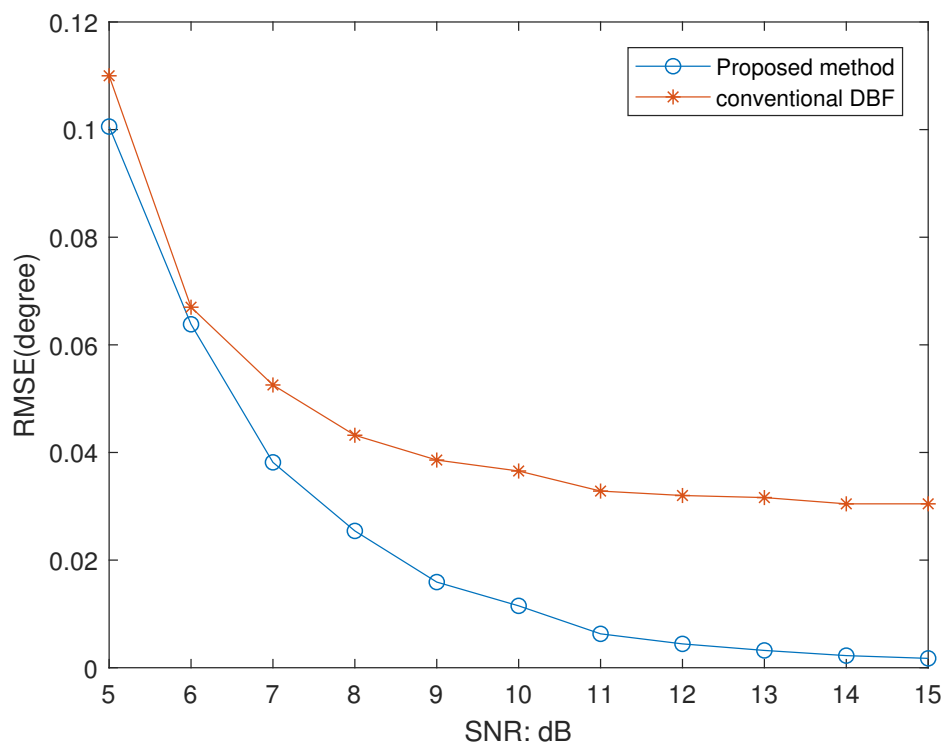

Figure 5.7: Root mean square error of 3 targets in the same range cell

In the second case, the tracking performance of MIMO-monopulse is evaluated. In this simulation, we generate single moving target and attempt to track it using MIMO-monopulse for several pulses. The target is initially located at 10 meters, $0^{\circ}$ to the radar, and moving perpendicularly to the broadside direction of the array. A beam scanning will be first performed to determine the angle of the target to generate sum and difference beams, and then in the following several pulses, the angle of the target is estimated by using error voltage and monopulse response curve (MRC), which is introduced in Chapter 3. During the tracking procedure, the sum and difference beams are fixed, and it's not necessary to repeat a digital beam scanning.

The estimation error of the proposed MIMO-monopulse is compared with both amplitude comparison and phase comparison methods. From this result, the estimation error of three methods are very similar initially, but after the target moves far away from the broadside direction, the estimation error of amplitude and phase comparison method become worse than our proposed method, because the monopulse ratio has better approximation of linear region.

Assume the velocity of a target to be $50 \mathrm{~m} / \mathrm{s}$, the angle shift is around 4 degrees within 128 pulses. Therefore, to guarantee the accuracy of estimation, a new beam should be steered to 4 degrees and continue to track the target. 


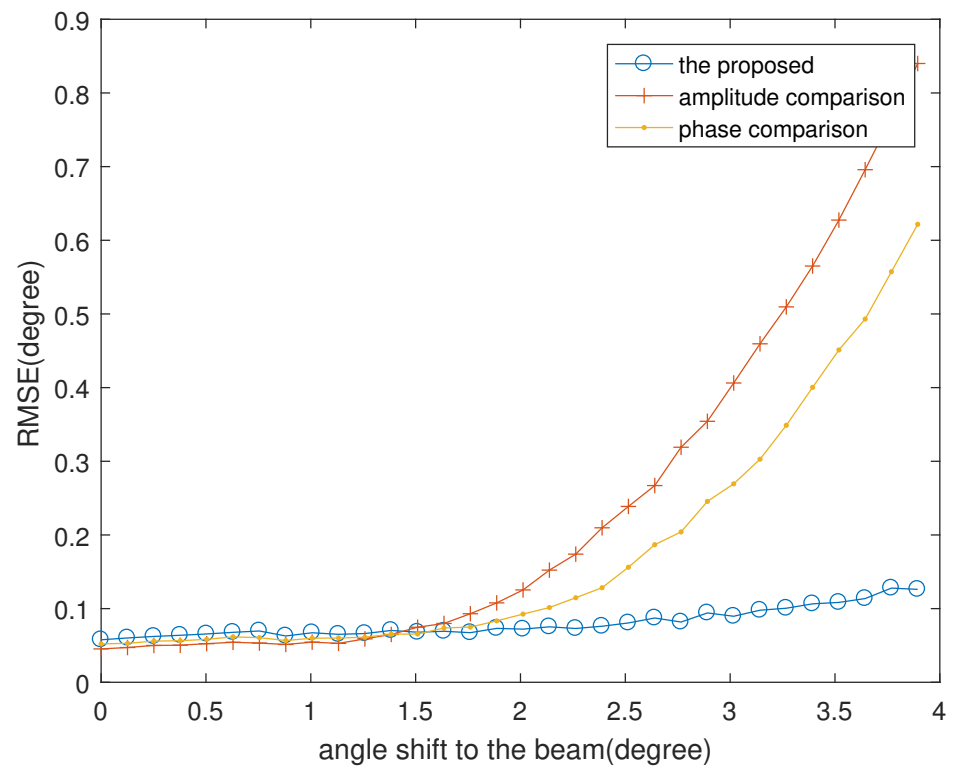

Figure 5.8: Root mean square error of tracking the target using MRC with fixed beam, SNR is 15dB and the number of pulses is 128

Consider the above comparisons, our proposed synthesis of sum and difference pattern has better performance in simulated scenarios.

\subsubsection{STAP MIMO-MONOPULSE}

Consider the effect of ground clutter, we add the simulation of clutter model into FMCW signals. Assume the velocity of the clutter is $15 \mathrm{~m} / \mathrm{s}$, which in realistic equals to the opposite velocity of the vehicle installing the automotive radar. The clutter model is constructed by generating clutter patch distributed in each range cell and all directions (step of angle is 1 degree). The distribution of clutter is assumed to be Rayleigh. The STAP processing of monopulse sum and difference beam is illustrated in Figure 5.9 and Figure 5.10. It can be seen that in the angle-Doppler map, the clutter generates a widespread in both angle and Doppler spectrum which has compatible response pattern with targets.In angular domain, it results in non-existed targets to be detected. After STAP processing, however, there will be a very deep nulling at the locations of ground clutter, thus the false detection is avoided. After obtaining both sum and difference beams using STAP, then we can calculate the monopulse ratio.

Due to the limitation of time, the idea of extending the MIMO-monopulse algorithm to STAP hasn't been verified by real experimental data. In the future work, STAP MIMO-monopulse will be further studied for the clutter suppression. 


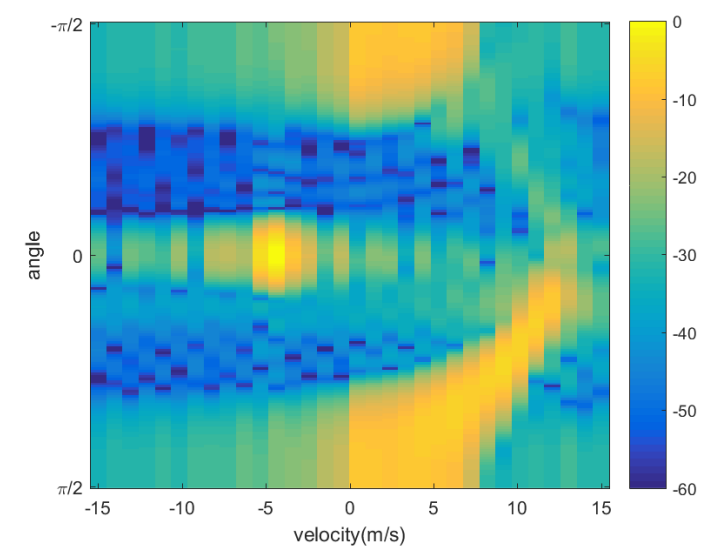

(a) Spatial-temporal sum beam without STAP

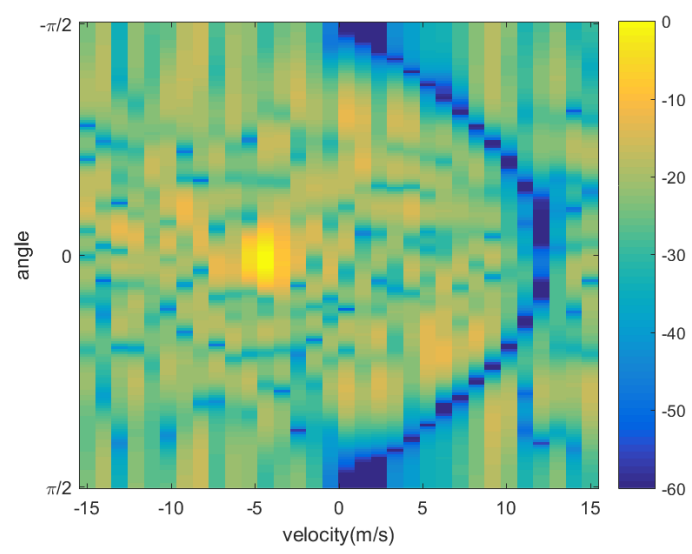

(b) STAP spatial-temporal sum beam

Figure 5.9: Angle-Doppler map of STAP MIMO-monopulse, sum beam

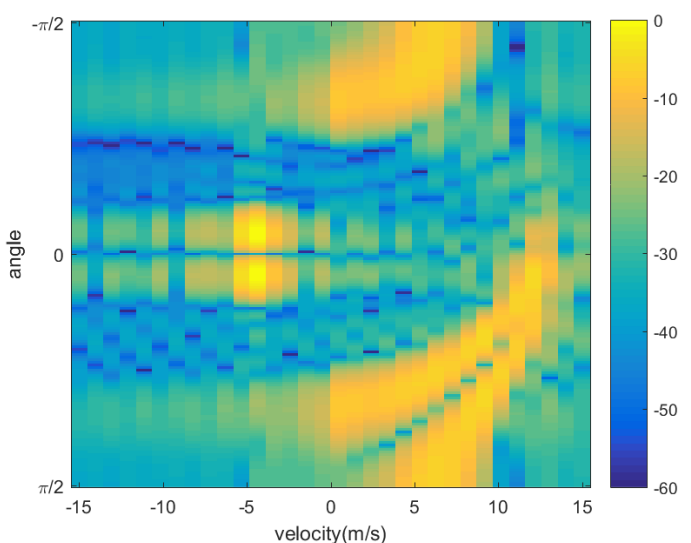

(a) Spatial-temporal difference beam without STAP

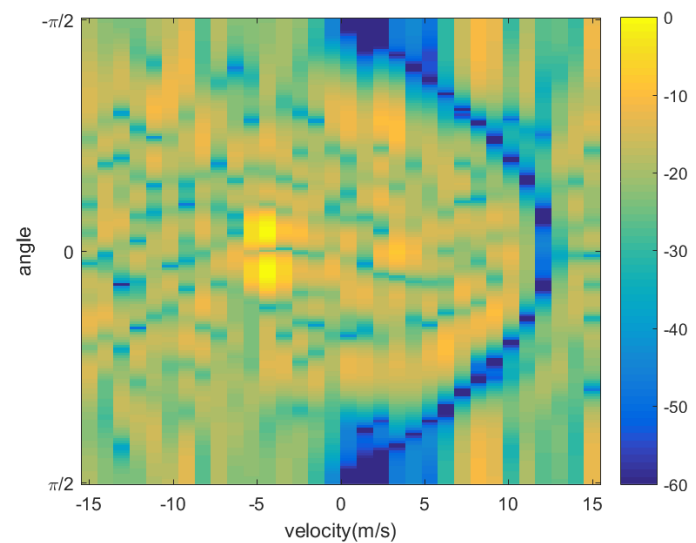

(b) STAP spatial-temporal difference beam

Figure 5.10: Angle-Doppler map of STAP MIMO-monopulse, difference beam

\subsection{Experimental Data Processing}

The MIMO-monopulse algorithm is tested on real data collected by NXP Dolphin automotive radar. The Dolphin test-chip (TC) is a single-chip automotive FMCW radar transceiver for short, medium and long-range radar applications, covering the full car-radar frequency band from $76 \mathrm{GHz}$ to $81 \mathrm{GHz}$. In our experiment, we use the center frequency of $79 \mathrm{GHz}$, which is required to be utilized for short range application.

Dolphin has 3 transmitter and 4 receiver elements, the distance between two receiver elements is $0.1899 \mathrm{~mm}$, which is equal to $\lambda / 2$ at $78.5 \mathrm{GHz}$. The spacing between 3 transmitters is as 3 times as the receiver, which guarantees that there are no grating lobes. The photo of the Dolphin is shown in Figure 5.11.

The radar is connected to a computer, which controls the radar through a software. The software is provided by NXP and developed by the software engineer in Microwave Sensing, Signals and Systems group. Measurement data is collected and restored in the standard format, and then processed by MATLAB code. 
NXP has also provided the preliminary processing MATLAB code, which realizes 3D FFT to get range, Doppler and DOA.

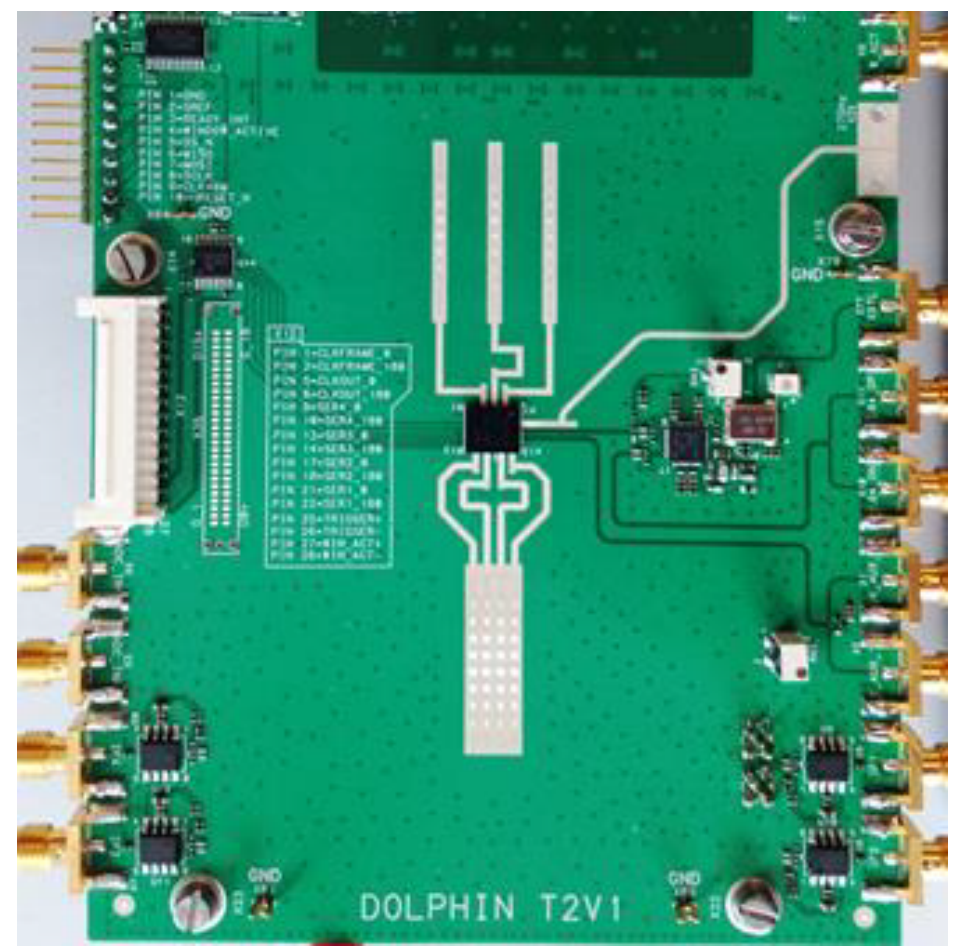

Figure 5.11: Transmitter(upper 3 elements) and Receiver(lower4 elements) array of Dolphin

\subsubsection{CALibration of MiMO ARray}

In DOA estimation, the calibration array is a crucial scheme, because all kinds of algorithms require accurately constructed steering vectors. However, the steering vector can suffer from random errors due to imperfect calibrated arrays, which often includes gain and phase uncertainty, mutual coupling, and sensor location errors. Gain and phase uncertainty is usually caused by differences among the cabling. To avoid grating lobes, antenna elements of the array are often placed close to generate electromagnetic interactions among sensors, called mutual couplings. Also, there may exist errors due to the inaccurate position of the antenna element. The amplitude and phase of each element in antenna array always have deviation which will result in the undesirable antenna pattern, hence, those errors/perturbations must be compensated in order to achieve precise steering vector [57].

The most common methods of calibration are based on the following model. Consider $N$ measurement of a virtual array with $M$ elements. The received antenna array vector for a signal incident from direction $\phi$ can be written as [58].

$$
\mathbf{b}(\theta)=\mathbf{C a}(\theta),
$$

where $\mathbf{b}$ is an $M \times 1$ vector of the measurement, $\mathbf{C}$ is an $M \times M$ matrix representing the gain/phase uncertainty and mutual coupling, and $\mathbf{a}(\phi)$ is an $M \times 1$ vector describing the received voltages.

According to 5.4, one needs to know only $\mathbf{C}$ to predict the antenna array steering vector for any direction of 
arrival. The simplest of these assume that the vector $\mathbf{b}(\phi)$ is measured for $M$ independent (widely separated and not coincident with grating lobes), directions such that $M>N$. Let these measurements be represented by the $N \times M$ matrix $\mathbf{B}=\left[\mathbf{b}_{1}, \mathbf{b}_{2}, \cdots, \mathbf{b}_{M}\right]$. Then the calibrated steering matrix can be rewritten as:

$$
\mathbf{B}=\mathbf{C A} \text {, }
$$

where $\mathbf{A}=\left[\mathbf{a}_{1}, \mathbf{a}_{2}, \cdots, \mathbf{a}_{M}\right]$ is an overdetermined set of linear simultaneous equations. The least square solution for $\mathbf{C}$ is given by:

$$
\mathbf{C}=\mathbf{B} \mathbf{A}^{H}\left(\mathbf{A} \mathbf{A}^{H}\right)^{-1} .
$$

If we consider mutual coupling, then in matrix $\mathbf{C}$, each column represents the mutual coupling factor between all pairs of the elements. In this thesis, due to the limitation of time we just ignore the interaction between pairs of the antenna elements, which leads to the matrix $\mathbf{C}$ to be diagonal. Then the equation 5.4 is simplified as

$$
\mathbf{C}(i, i)=\frac{\mathbf{B}_{\theta=0}(i)}{\mathbf{A}_{\theta=0}(i)} \quad, i=1, \cdots, M,
$$

where $\mathbf{B}_{\theta=0}$ is single measurement and $\mathbf{A}_{\theta=0}$ is the ideal steering vector at $\theta$. The diagonal matrix from the above equation just calibrates the gain and phase error of the MIMO array. In NXP processing code, a calibration matrix $\mathbf{C}$ is already given. However in the course of the experiments, we have found the performance of calibration is not ideal, thus we make a new experiment to calibrate the MIMO array.

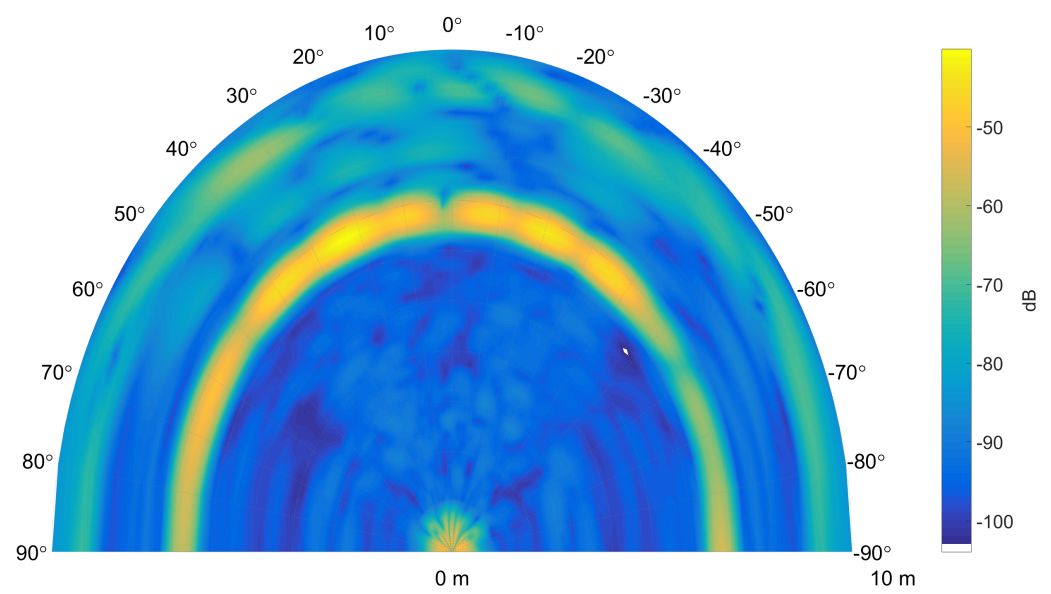

(a) Range azimuth map without calibration 


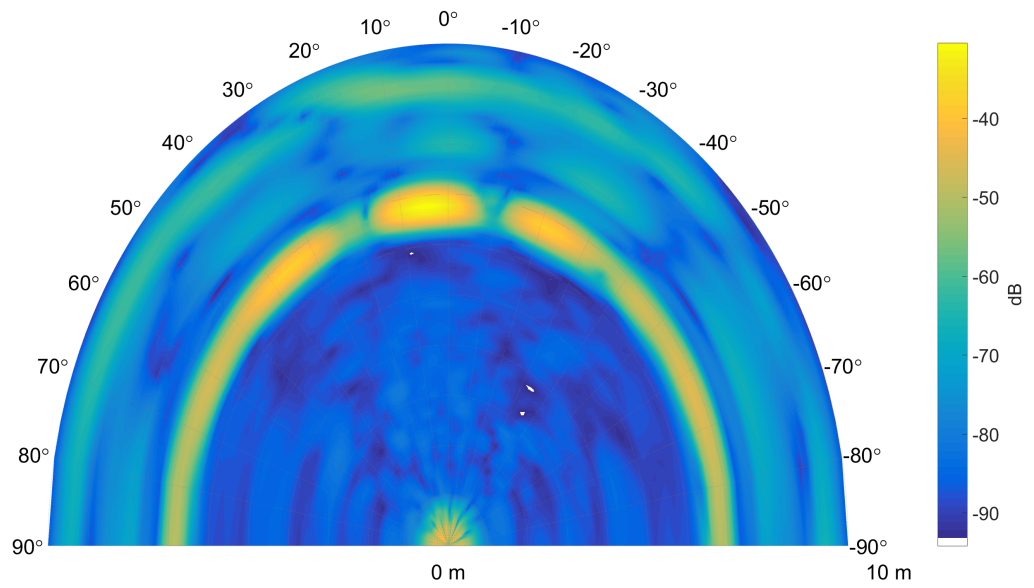

(b) Range azimuth map with NXP's calibration

Figure 5.12: Range azimuth map, target at $6.8 \mathrm{~m}$ and $0^{\circ}$.

In our experiment, a flat plate target located at 6.8 meters and the angle to the radar is 0 is measured. Firstly, we use FFT on range and DBF on the angle to obtain the 2D range-azimuth map. As illustrated in figure 5.12, after calibration using NXP samples the main beam is shifted but still has some bias to the direction of the target (around $10^{\circ}$, the target should be at $0^{\circ}$ ). Also, the nulling of the DBF pattern is not as expected.

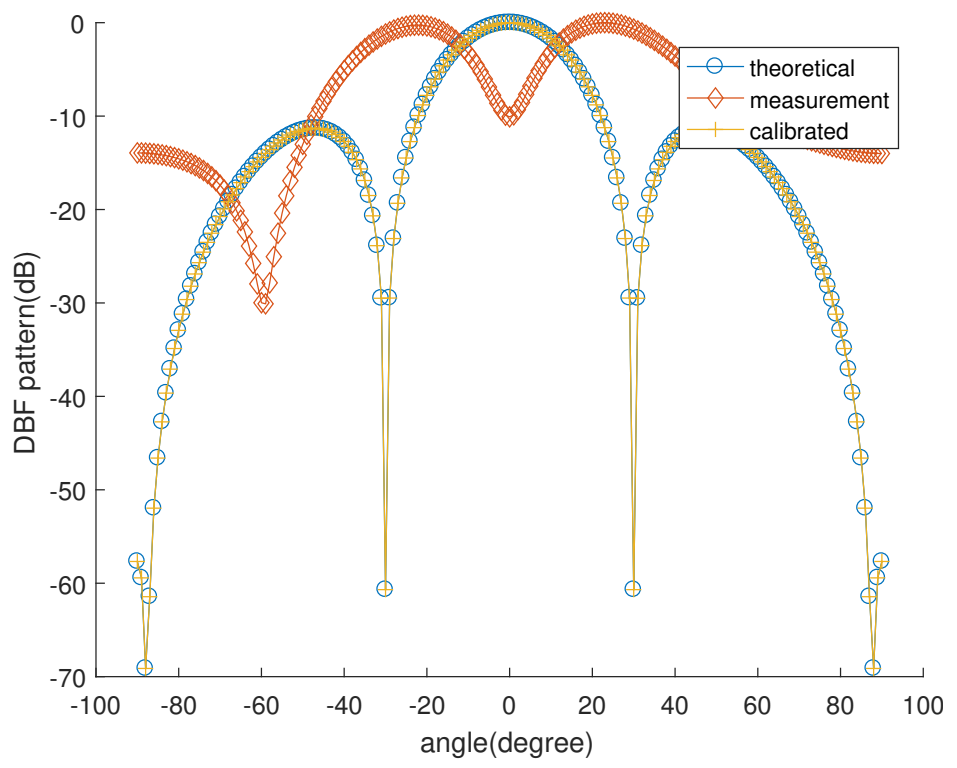

(a) Calibrated pattern at $0^{\circ}$ 


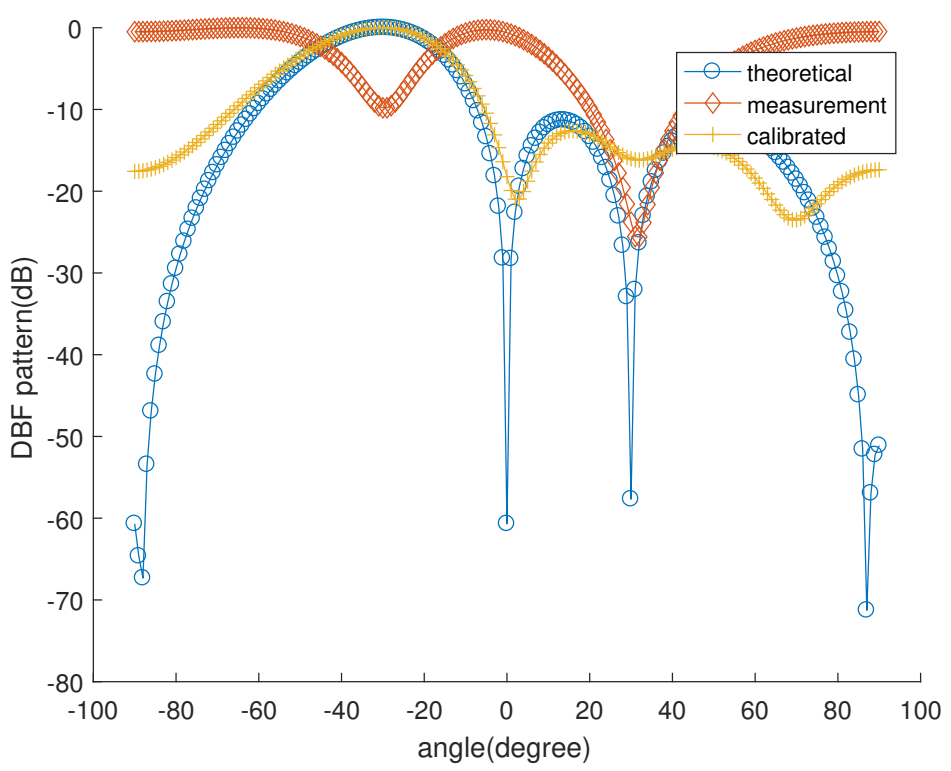

(b) Calibrated pattern at $-30^{\circ}$

Figure 5.13: Calibration of receiver

We extract the data at the range cell of the target (6.8 meters) at $0^{\circ}$ as measurement vector $\mathbf{b}$ to compute the diagonal calibration matrix. Firstly, we consider calibrating the receiver array. Since the measurement data is stored in 12 channels, we take the received signals from the same transmitter, thus we have three $4 \times 1$ measurement vector. For each of them, calibration is realized using equation 5.7 , and the result is shown in Figure 5.13.

Similarly, transmitter steering vector received by each receiving channel is calibrated and the result is shown in Figure 5.14. Notice that since the transmitter inter-element spacing is $3 \lambda / 2$, there are 2 grating lobes. From the calibration result of transmitter and receiver array, it can be seen that the mutual coupling effect of the transmitter is very weak, thus the calibrated antenna pattern is well fixed the theoretical pattern. The mutual coupling effect of receiver pattern is relatively strong as the nulling of the pattern is not precisely located such as illustrated in Figure 5.13 (b). However, since the main beam is correctly steered to the desired angle, for MIMO-monopulse such a calibration result is acceptable. 


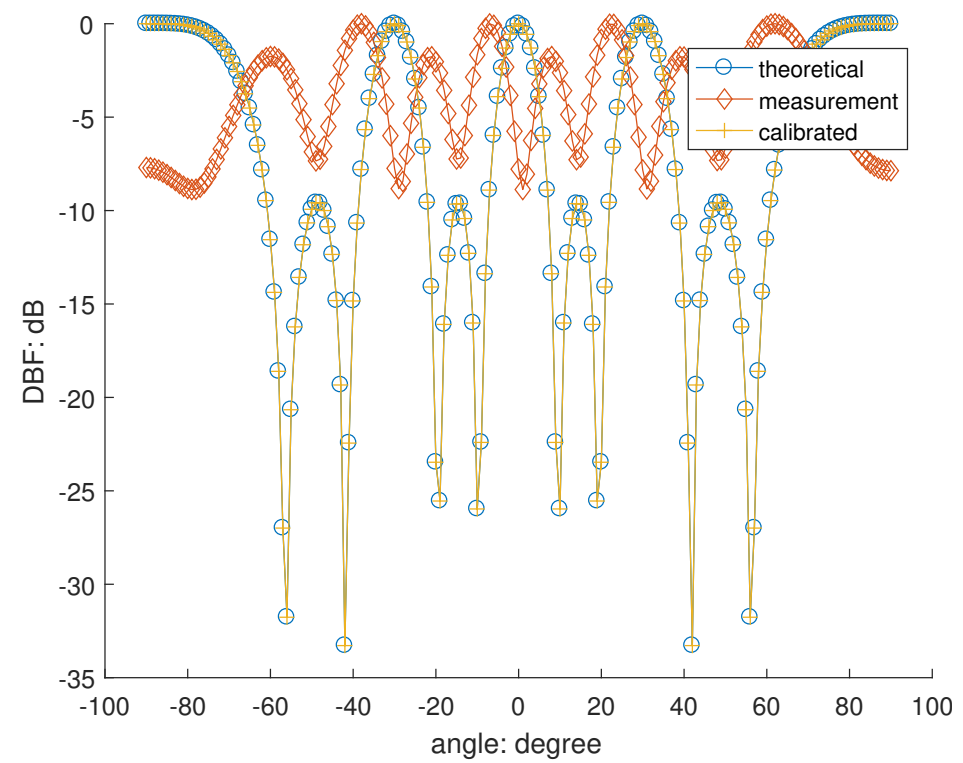

(a) Calibration at $0^{\circ}$

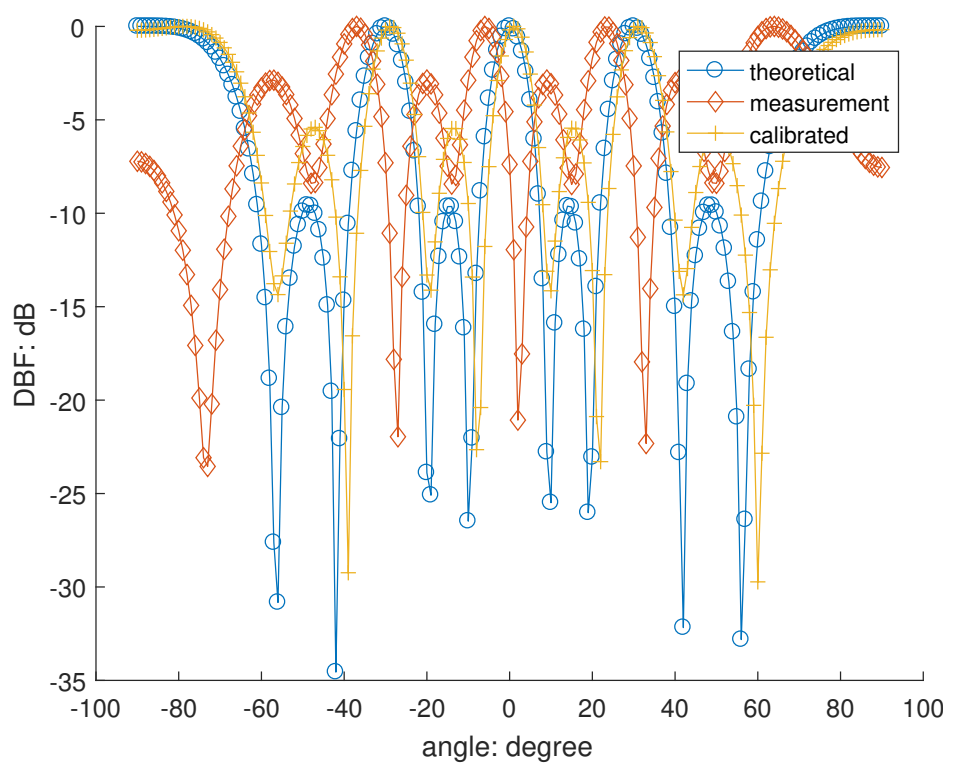

(b) Calibration at $-30^{\circ}$

Figure 5.14: Range azimuth map, target at $6.8 \mathrm{~m}$ and $0^{\circ}$. (a) is the measurement at $0^{\circ}$ and (b) is after calibration using NXP samples. 


\begin{tabular}{lc}
\hline Channel & Value \\
\hline 1 & $0.0128+0.1034 \mathrm{i}$ \\
2 & $0.1178+0.1354 \mathrm{i}$ \\
3 & $-0.0567-0.1022 \mathrm{i}$ \\
4 & $-0.0923-0.1740 \mathrm{i}$ \\
5 & $-0.0012+0.0976 \mathrm{i}$ \\
6 & $0.0821+0.1158 \mathrm{i}$ \\
7 & $-0.0213-0.1079 \mathrm{i}$ \\
8 & $-0.0374-0.1577 \mathrm{i}$ \\
9 & $-0.0312-0.0864 \mathrm{i}$ \\
10 & $-0.0753-0.1046 \mathrm{i}$ \\
11 & $0.0983+0.1323 \mathrm{i}$ \\
12 & $0.0627+0.1936 \mathrm{i}$ \\
\hline
\end{tabular}

Table 5.2: Calibration samples of each channel in virtual array

The range-azimuth map after calibration is shown in Figure 5.15 (a). We test the performance of several measurements taken on the different angle to the target, which is shown in 5.15 (b) and (c). The conclusion is that $\mathbf{C}$ obtained by measurement at $0^{\circ}$ can be applied to all measurement data.

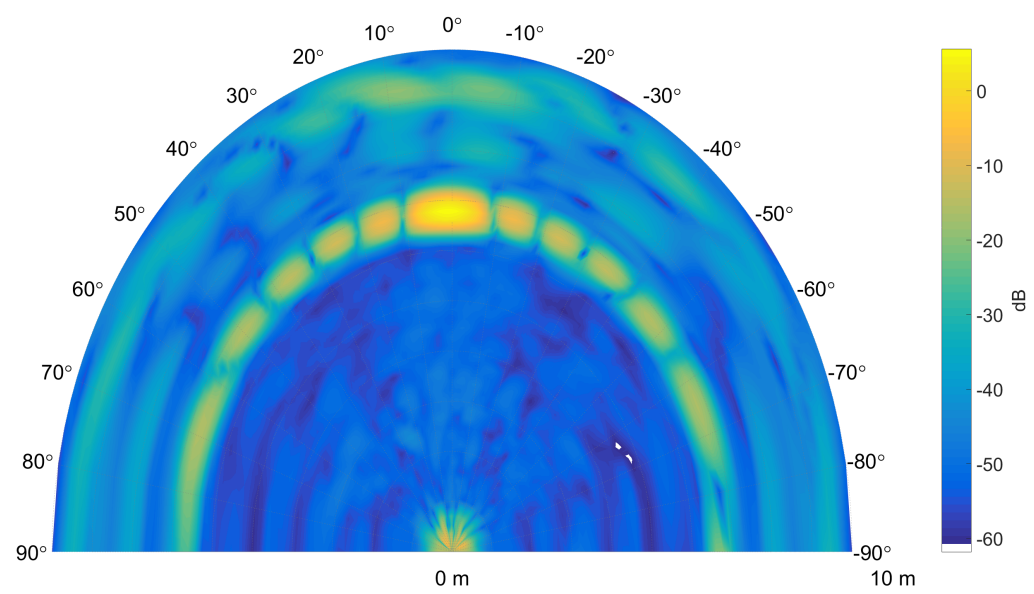

(a) Range azimuth map with the proposed calibration 


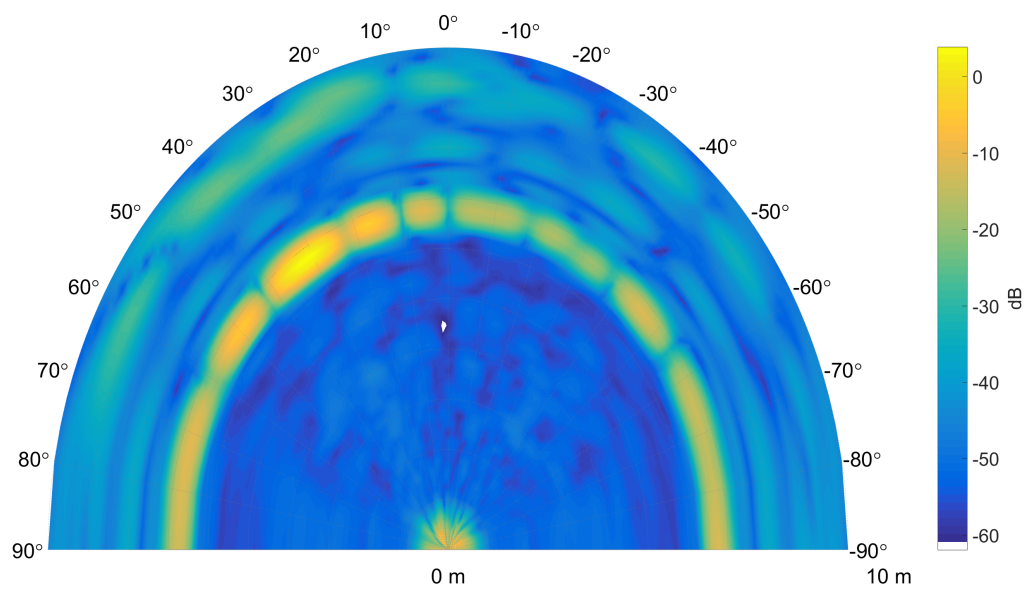

(b) Target at $-30^{\circ}$

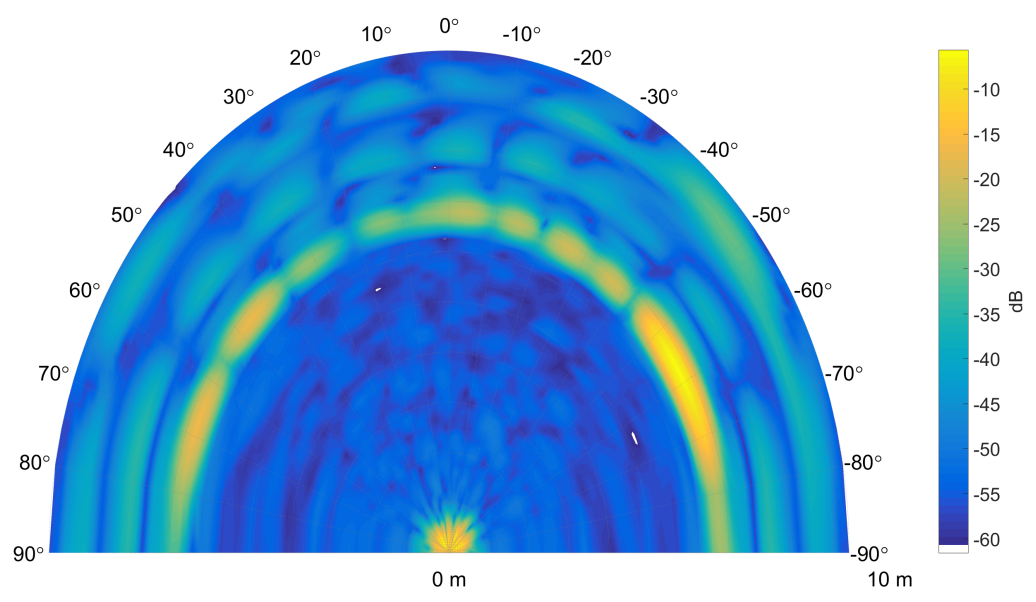

(c) Target at $60^{\circ}$

Figure 5.15: Range azimuth (DBF) after the proposed calibration, and test on different data sets

\subsubsection{Moving TARget DOA Estimation using MiMO-Monopulse}

The performance of MIMO-monopulse DOA algorithm is validated in a moving targets experiment. Two corner reflector is moving diagonally in the view of sight, opposite to each other. The measurement is made in an indoor environment within around 9 meters and the azimuth area is from $-20^{\circ}$ to $20^{\circ}$ to the radar. According to the definition of monopulse, the output of the estimation is a ratio which is exactly zero at the DOA of the target, which has a very deep nulling if it is illustrated in $\mathrm{dB}$. To compare the performance of MIMO-monopulse with conventional DBF in a simlar range-azimuth map, we just visualize it by taking the opposite, which generates a peak at the angle of the target. The result of processing real data is illustrated in Figure 5.16. It can be seen that compare with DBF, the estimation of MIMO-monopulse yields much more narrow 'spectrum'. The $-3 \mathrm{~dB}$ point of DBF pattern is around 4 degree, while for monopulse it is less than 0.1 degree, which means it has very sensitive azimuth determination. 


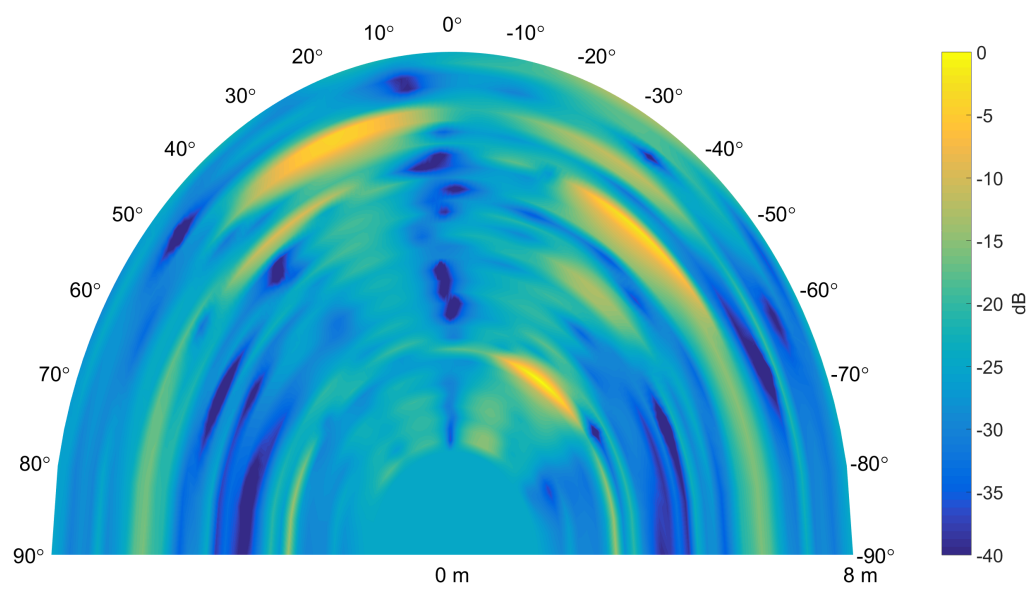

(a) Range azimuth map using FFT

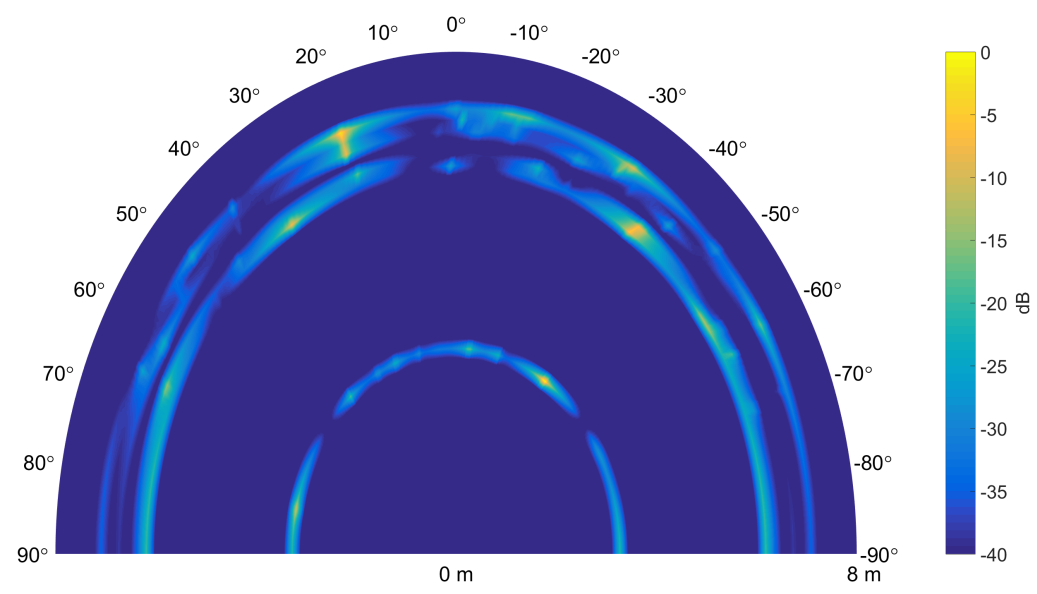

(b) Range-azimuth using MIMO-monopulse

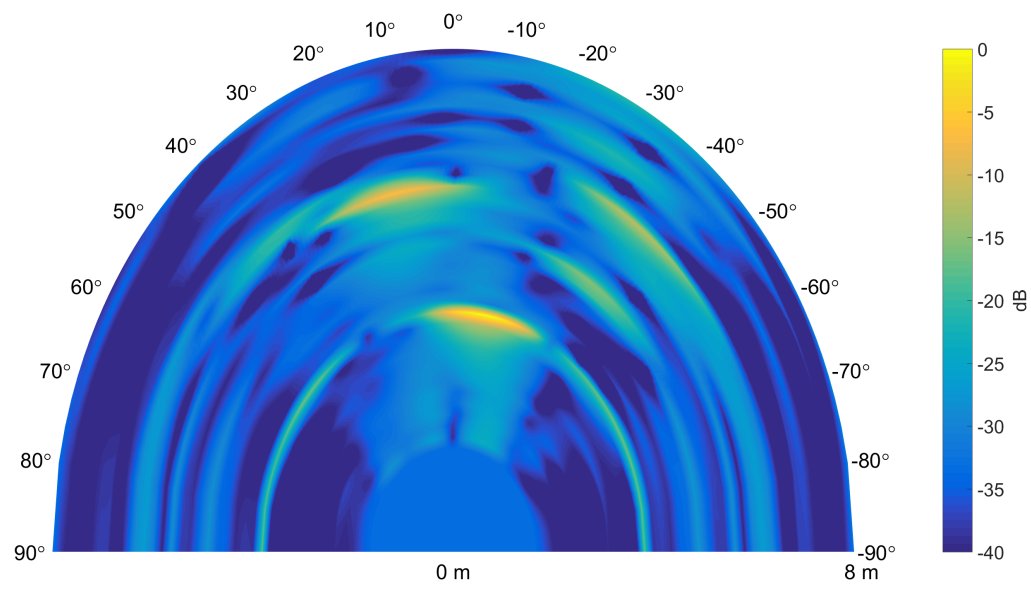

(c) Range azimuth map using FFT 


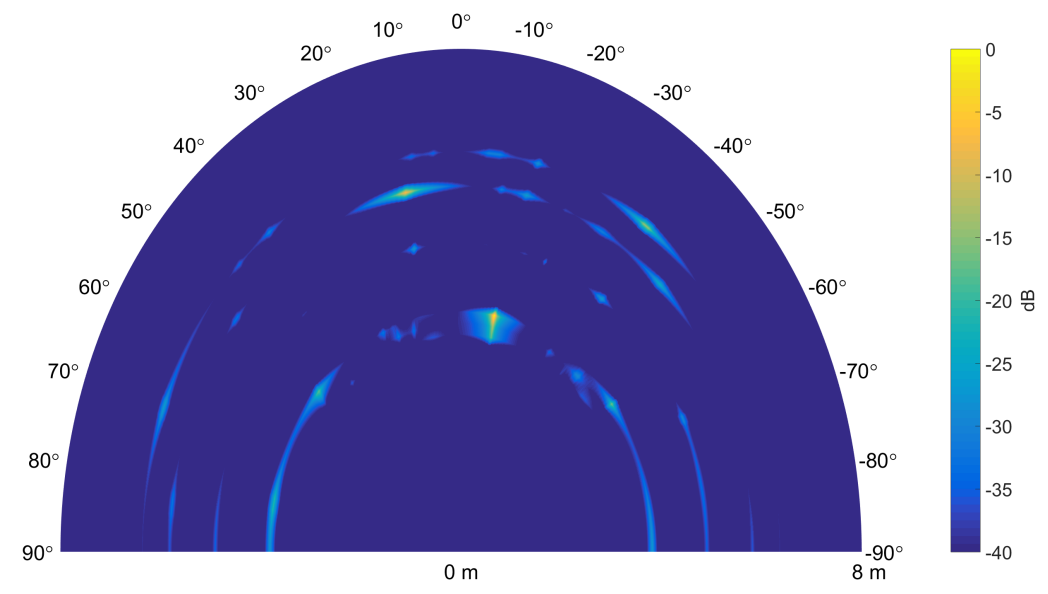

(d) Range-azimuth using MIMO-monopulse

Figure 5.16: Range azimuth maps: Comparison using MIMO-monopulse and traditional method 



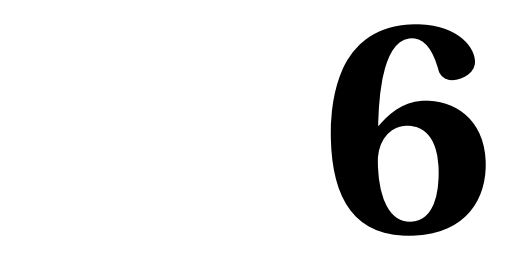

\section{CONCLUSION}

In this thesis, we propose the monopulse technique on the automotive radar for precise and rapid DOA estimation.

In order to achieve this goal, an FMCW MIMO signal model is constructed, which consists of fast-time, slow time and spatial samples. 3-dimensional processing is used to obtain the range, Doppler and angle of the targets. Particularly, MIMO-monopulse is expected to be applied on spatial samples to realize DOA estimation. Digital beamforming is implemented on the virtual array under the assumption of narrowband signals, while the effect of the wideband issue is also considered if the full $4 \mathrm{GHz}$ band is utilized, and the frequency dispersion caused by wideband is solved using the time-varying weighting vector.

We firstly consider conventional amplitude and phase comparison method to generate sum and difference beam for MIMO-monopulse. The implementation of sum and difference beam is based on the steering vector of a virtual array and classical digital beamforming. Basically, the amplitude comparison method utilizes the difference of amplitude response generated by two squinted beams steered to left and right, while the phase comparison manner pays attention to the phase difference of reflected wave to two phase centers of the left and right sub-arrays.

In this thesis, we utilize the synthesis of sum and difference pattern using Chebyshev and Zolotarev weighting, which is approved to provide equal level sidelobe reduction for both the sum and difference patterns and create a deep nulling at looking direction of the antenna array. To visualize the monopulse output in a range-azimuth map, we proposed a novel processing approach through mapping the monopulse ratio to DBF response pattern. Furthermore, we resolved the monopulse ratio ambiguity issue when applying monopulse technique on MIMO antenna configuration, which is never considered in previous literature and study. Last but not least, to implement the DOA estimation, we successfully calibrate the given MIMO array to realize the proposed algorithm in experimental setup. 
As a extension of the novel MIMO-monopulse method, we combine the MIMO-monopulse with adaptive processing to suppress the interference like jammer and clutter. Continuous waveform (CW) signals with a wide spread spectrum as the jamming signals are simulated to introduce the concept of adaptive digital beamforming.

In the automotive application, ground clutter is the visible interference to the DOA estimation problem, especially for the target with low radar cross section (RCS), such as a pedestrian. In this thesis, we extend MIMO-monopulse to 2-dimensional space-time adaptive processing (STAP), which enables to suppress the ground clutter in the angle-Doppler domain. Moreover, due to the usage of MIMO array, it is also possible to cancel multi-path clutter, while maintaining the DOA and Doppler shift of the target.

The proposed algorithm is simulated through MATLAB and verified using real data collected by NXP automotive radar. The MIMO-monopulse algorithm has shown better performance than conventional digital beamforming since it has a sharper peak on the main beam. Although the MIMO-monopulse and DBF hold the same angular resolution depending on the size of the array aperture, the azimuth determination accuracy is largely improved, as is discussed in Section 5.1.4. It also has advantages of the number of targets not necessary to be known, the capability to work in a single snapshot, nice performance in low SNR scenarios and very low computation time cost, compared with subspace-based super-resolution algorithms. Owing to the properties of tracking function, the proposed MIMO-monopulse target localization method shows good performance on the application of small targets tracking, e.g. pedestrian motion tracking, which is attractive in the automotive radar application.

\subsection{Future WORK}

In this thesis, MIMO-monopulse has already shown desired performance in DOA estimation of FMCW automotive radar. But there still remains some problems need to be solved to improve the algorithm.

MIMO-monopulse algorithm relies on the previous range processing and detection because the output of monopulse cannot discriminate the range of the targets. Constant false alarm rate (CFAR) detection is commonly applied to detect the target on range domain. In a practical realization of the algorithm, it might be hard to determine the parameters of CFAR, which may lead to the wrong detection of the target. One possible solution to this problem is to consider two dimension estimation algorithm, which can jointly estimate the range and angle of the target. Basically, the idea is to reshape the spatial-time matrix into a vector and process it using MIMO-monopulse. In this thesis, to process the actual radar data without false detection using CFAR, we attempt to normalize the monopulse ratio in $\mathrm{dB}$ by using sum pattern (discussed in Section 5.1.4), since the utilize of sum pattern provides information of range.

As is discussed in chapter 5, the MIMO-monopulse has the ambiguity of monopulse ratio in $\mathrm{dB}$ caused by sidelobe zero crossing. In this thesis, our proposed method is to suppress the sidelobe ratio using a scalar which is carried out by normalizing the sum pattern. After this processing, the deep nulling of monopulse ratio in $\mathrm{dB}$ is kept exactly at the direction of the target. It is still potential to think of other methods to solve this ambiguity in MIMO-monopulse. 
As an extension of MIMO-monopulse, we considered STAP to suppress the common road clutter and multi-path clutter in the angle-Doppler domain. The problem of STAP MIMO-monopulse is that the covariance matrix needs to be estimated, and the increased degrees of freedom requires a large amount of training data to estimate the covariance matrix. Several reduced dimension STAP has been published in some literature. How to reduce the required number of training data is attractive, especially in automotive radar application where sometimes limited number of measurement is available. Besides, due to the limit of time, we just built a simulation model of clutter suppression by using STAP MIMO-monopulse. The experimental data will be checked in the future to verify the clutter model, and the performance of suppressing ground and multi-path clutter will be tested.

In short range automotive application, the full bandwidth is $4 \mathrm{GHz}$. Although in our experiment the radar covers only $1 \mathrm{GHz}$, it is still attractive to research the effect of wideband (the application of MIMOmonopulse may not be limited to automotive radar). Conventional wideband beamforming includes tappeddelay-lines(TDLs) filter, sub-banding algorithm and Fourier domain beamforming. In our thesis, to take the advantage of linear FMCW modulation, we simply apply time-varying weighting vector for DBF based on a convenient coupling between time and frequency. The dispersion of the main lobe is avoided through timevarying weighting, however, the dispersion of interference is not considered(e.g. jammer and clutter), which will affect the adaptive processing. One possible solution of wideband adaptive beamforming for LFM signal is to subdivide the received chirp signal into segments and then recompute the adaptive weights within each segment using the segment data to train the adaptive weights. However, this method will limit the number of training samples in each segment [59]. Better solution of wideband adaptive beamforming for FMCW signals is still potential to be studied in the future.

The performance of MIMO-monopulse is expected to be validated on the application of tracking pedestrian in a real experiment. Currently, the algorithm is only tested in an indoor environment using ideal reflector as moving targets. To estimate the angle of pedestrian might be more complex, radar cross section(RCS) model of the human in $79 \mathrm{GHz}$ center frequency and $4 \mathrm{GHz}$ bandwidth is possible to be analyzed.

In this thesis, we extend the monopulse technique on MIMO radar for target localization. It is still possible to consider other improvements of monopulse. The strategy can be to optimize the topology of the antenna array for monopulse. In [60], some different topology of MIMO array is compared to improve the performance of wideband imaging radar. Some of those of topology can also be considered for a MIMOmonopulse array, for example, optimize the transmitter array for generating beams with low sidelobe level to actively scan using sequential beams. This could be combined with MIMO-monopulse radar through detection using transmitter scanning beam and then localizing the target by MIMO-monopulse. 



\section{BIBLIOGRAPHY}

[1] D. Kissinger, Millimeter-wave receiver concepts for $77 \mathrm{GHz}$ automotive radar in silicon-germanium technology. Springer Science \& Business Media, 2012.

[2] J. Wenger, "Automotive radar-status and perspectives," in Compound Semiconductor Integrated Circuit Symposium, 2005. CSIC'05. IEEE, pp. 4-pp, IEEE, 2005.

[3] J.-J. Lin, Y.-P. Li, W.-C. Hsu, and T.-S. Lee, “Design of an FMCW radar baseband signal processing system for automotive application," SpringerPlus, vol. 5, no. 1, p. 42, 2016.

[4] V. Winkler, "Range Doppler detection for automotive FMCW radars," in Microwave Conference, 2007. European, pp. 1445-1448, IEEE, 2007.

[5] J. Hasch, E. Topak, R. Schnabel, T. Zwick, R. Weigel, and C. Waldschmidt, "Millimeter-wave technology for automotive radar sensors in the $77 \mathrm{GHz}$ frequency band," IEEE Transactions on Microwave Theory and Techniques, vol. 60, no. 3, pp. 845-860, 2012.

[6] W.-Q. Wang, H. C. So, and A. Farina, "An overview on time/frequency modulated array processing," IEEE Journal of Selected Topics in Signal Processing, 2016.

[7] H. Zhou, P. Cao, and S. Chen, "A novel waveform design for multi-target detection in automotive FMCW radar," in Radar Conference (RadarConf), 2016 IEEE, pp. 1-5, IEEE, 2016.

[8] Y. Fan, K. Xiang, J. An, and X. Bu, “A new method of multi-target detection for FMCW automotive radar," 2013.

[9] E. Hyun and J.-H. Lee, "A meothod for multi-target range and velocity detection in automotive FMCW radar," in Intelligent Transportation Systems, 2009. ITSC'09. 12th International IEEE Conference on, pp. 15, IEEE, 2009.

[10] J. Li and P. Stoica, "MIMO radar with colocated antennas," IEEE Signal Processing Magazine, vol. 24, no. 5, pp. 106-114, 2007.

[11] A. M. Haimovich, R. S. Blum, and L. J. Cimini, “MIMO radar with widely separated antennas," IEEE Signal Processing Magazine, vol. 25, no. 1, pp. 116-129, 2008.

[12] H. Li and B. Himed, “Transmit subaperturing for MIMO radars with co-located antennas," IEEE Journal of Selected Topics in Signal Processing, vol. 4, no. 1, pp. 55-65, 2010. 
[13] J. Du Preez and S. Sinha, Millimeter-Wave Antennas: Configurations and Applications. Springer, 2016.

[14] J. De Wit, W. Van Rossum, and A. De Jong, “Orthogonal waveforms for FMCW MIMO radar," in Radar Conference (RADAR), 2011 IEEE, pp. 686-691, IEEE, 2011.

[15] G. Babur, O. A. Krasnov, A. Yarovoy, and P. Aubry, "Nearly orthogonal waveforms for MIMO FMCW radar," IEEE Transactions on Aerospace and Electronic Systems, vol. 49, no. 3, pp. 1426-1437, 2013.

[16] S. Fortunati, R. Grasso, F. Gini, M. S. Greco, and K. LePage, "Single-snapshot DOA estimation by using compressed sensing," EURASIP Journal on Advances in Signal Processing, vol. 2014, no. 1, p. 120, 2014.

[17] H. Krim and M. Viberg, "Two decades of array signal processing research: the parametric approach," IEEE signal processing magazine, vol. 13, no. 4, pp. 67-94, 1996.

[18] J. Bohme, "Estimation of source parameters by maximum likelihood and nonlinear regression," in Acoustics, Speech, and Signal Processing, IEEE International Conference on ICASSP'84., vol. 9, pp. 271274, IEEE, 1984.

[19] A. G. Jaffer, "Maximum likelihood direction finding of stochastic sources: A separable solution," in Acoustics, Speech, and Signal Processing, 1988. ICASSP-88., 1988 International Conference on, pp. 28932896, IEEE, 1988.

[20] G. O. Manokhin, Z. T. Erdyneev, A. A. Geltser, and E. A. Monastyrev, "Music-based algorithm for rangeazimuth FMCW radar data processing without estimating number of targets," in Microwave Symposium (MMS), 2015 IEEE 15th Mediterranean, pp. 1-4, IEEE, 2015.

[21] D. Oh, Y. Ju, H. Nam, and J.-H. Lee, “Dual smoothing DOA estimation of two-channel FMCW radar," IEEE Transactions on Aerospace and Electronic Systems, vol. 52, no. 2, pp. 904-917, 2016.

[22] M. Schoor and B. Yang, "High-resolution angle estimation for an automotive FMCW radar sensor," in Proc. Intern. Radar Symposium (IRS), 2007.

[23] T. K. Sarkar and O. Pereira, "Using the matrix pencil method to estimate the parameters of a sum of complex exponentials,” IEEE Antennas and Propagation Magazine, vol. 37, no. 1, pp. 48-55, 1995.

[24] D. Oh and J.-H. Lee, "Low-complexity range-azimuth FMCW radar sensor using joint angle and delay estimation without svd and evd," IEEE Sensors Journal, vol. 15, no. 9, pp. 4799-4811, 2015.

[25] S. M. Sherman and D. K. Barton, Monopulse principles and techniques. Artech House, 2011.

[26] U. Nickel, “Overview of generalized monopulse estimation,” IEEE Aerospace and Electronic Systems Magazine, vol. 21, no. 6, pp. 27-56, 2006.

[27] P. Molchanov, S. Gupta, K. Kim, and K. Pulli, "Short-range FMCW monopulse radar for hand-gesture sensing," in Radar Conference (RadarCon), 2015 IEEE, pp. 1491-1496, IEEE, 2015. 
[28] G. Gottardi, N. Ebrahimi, P. Rocca, and A. Massa, "Optimal synthesis of monopulse beamforming weights for airborne radars through convex optimization," in Applied Computational Electromagnetics Society Symposium-Italy (ACES), 2017 International, pp. 1-2, IEEE, 2017.

[29] G. Gottardi, L. Poli, P. Rocca, A. Montanari, A. Aprile, and A. Massa, "Optimal monopulse beamforming for side-looking airborne radars," IEEE Antennas and Wireless Propagation Letters, vol. 16, pp. 12211224, 2017.

[30] U. Nickel, "Performance analysis of space-time-adaptive monopulse," Signal Processing, vol. 84, no. 9, pp. 1561-1579, 2004.

[31] R. Klemm and U. Nickel, “Adaptive monopluse with STAP,” in Radar, 2006. CIE'06. International Conference on, pp. 1-4, IEEE, 2006.

[32] Y. Seliktar, D. B. Williams, and E. J. Holder, “A space/fast-time adaptive monopulse technique," EURASIP Journal on Advances in Signal Processing, vol. 2006, no. 1, p. 014510, 2006.

[33] S. Gogineni and A. Nehorai, "Monopulse MIMO radar for target tracking," IEEE Transactions on Aerospace and Electronic Systems, vol. 47, no. 1, pp. 755-768, 2011.

[34] S. Gogineni and A. Nehorai, "Target tracking using monopulse MIMO radar with distributed antennas," in Radar Conference, 2010 IEEE, pp. 194-199, IEEE, 2010.

[35] J. Zhang, H. Liu, J. Li, and C. Han, "Research on the mono-pulse phase comparison angle measurement algorithm of MIMO radar," in Microwave and Millimeter Wave Circuits and System Technology (MMWCST), 2012 International Workshop on, pp. 1-4, IEEE, 2012.

[36] J. Li, Z. Wang, H. Liu, Z. He, and J. Zhang, "Analysis of angular accuracy of amplitude comparison monopulse angle measurement for MIMO radar," in The Proceedings of the Third International Conference on Communications, Signal Processing, and Systems, pp. 245-252, Springer, 2015.

[37] T. Ito, K. Hirata, and R. Takahashi, "Transmitting phase comparison monopulse estimation using MIMO radar technique for distributed array," in Antennas and Propagation (ISAP), 2012 International Symposium on, pp. 78-81, IEEE, 2012.

[38] F. Uysal, M. Yeary, N. Goodman, R. F. Rincon, and B. Osmanoglu, "Waveform design for wideband beampattern and beamforming," in Radar Conference (RadarCon), 2015 IEEE, pp. 1062-1066, IEEE, 2015.

[39] H. L. Van Trees, Detection, estimation, and modulation theory. John Wiley \& Sons, 2004.

[40] H. L. Van Trees, Optimum array processing: Part IV of detection, estimation and modulation theory, vol. 1. Wiley Online Library, 2002.

[41] S. Weiss, R. W. Stewart, M. Schabert, I. K. Proudler, and M. W. Hoffman, "An efficient scheme for broadband adaptive beamforming," in Signals, Systems, and Computers, 1999. Conference Record of the ThirtyThird Asilomar Conference on, vol. 1, pp. 496-500, IEEE, 1999. 
[42] C.-h. An, J. Yang, R. Ran, U. Y. Pak, Y.-J. Ryu, and D. K. Kim, "Enhanced monopulse MIMO radar using reliable $\alpha \beta$ filtering," in MILITARY COMMUNICATIONS CONFERENCE, 2012-MILCOM 2012, pp. 1-6, IEEE, 2012.

[43] B. R. Mahafza, Introduction to radar analysis. CRC press, 1998.

[44] W. Rowe, M. Ström, J. Li, and P. Stoica, "Robust adaptive beamforming for MIMO monopulse radar," in SPIE Defense, Security, and Sensing, pp. 87140P-87140P, International Society for Optics and Photonics, 2013.

[45] Y. Seliktar, Space-time adaptive monopulse processing. PhD thesis, Georgia Institute of Technology, 1998.

[46] C. Dolph, "A current distribution for broadside arrays which optimizes the relationship between beam width and side-lobe level," Proceedings of the IRE, vol. 34, no. 6, pp. 335-348, 1946.

[47] R. J. Stegen, "Excitation coefficients and beamwidths of Tschebyscheff arrays," Proceedings of the IRE, vol. 41, no. 11, pp. 1671-1674, 1953.

[48] F. J. Harris, "On the use of windows for harmonic analysis with the discrete Fourier transform," Proceedings of the IEEE, vol. 66, no. 1, pp. 51-83, 1978.

[49] O. Price and R. Hyneman, "Distribution functions for monopulse antenna difference patterns," IRE Transactions on Antennas and Propagation, vol. 8, no. 6, pp. 567-576, 1960.

[50] D. McNamara, "Direct synthesis of optimum difference patterns for discrete linear arrays using Zolotarev distributions," in IEE Proceedings H-Microwaves, Antennas and Propagation, vol. 140, pp. 495-500, IET, 1993.

[51] A. Willis and C. Baker, "Approximation to Zolotarev polynomial ideal difference beams for linear arrays," Electronics letters, vol. 42, no. 10, pp. 561-563, 2006.

[52] J. Yu and J. Krolik, "MIMO multipath clutter mitigation for gmti automotive radar in urban environments," 2012.

[53] G. Hickman and J. L. Krolik, “MIMO GMTI radar with multipath clutter suppression,” in Sensor Array and Multichannel Signal Processing Workshop (SAM), 2010 IEEE, pp. 65-68, IEEE, 2010.

[54] W. L. Melvin and J. A. Scheer, Principles of modern radar. SciTech Publishing, 2014.

[55] I. S. Reed, J. D. Mallett, and L. E. Brennan, “Rapid convergence rate in adaptive arrays," IEEE Transactions on Aerospace and Electronic Systems, no. 6, pp. 853-863, 1974.

[56] P. Häcker and B. Yang, “Single snapshot DOA estimation,” Advances in Radio Science, vol. 8, pp. 251-256, 2010.

[57] H. Liu, L. Zhao, Y. Li, X. Jing, and T.-K. Truong, "A sparse-based approach for DOA estimation and array calibration in uniform linear array," IEEE sensors journal, vol. 16, no. 15, pp. 6018-6027, 2016. 
[58] I. J. Gupta, J. R. Baxter, S. W. Ellingson, H.-G. Park, H. S. Oh, and M. G. Kyeong, "An experimental study of antenna array calibration,” IEEE Transactions on antennas and propagation, vol. 51, no. 3, pp. 664-667, 2003.

[59] M. A. Richards, J. A. Scheer, W. A. Holm, B. Beckley, P. Mark, A. Richards, et al., "Principles of modern radar volume i-basic principles," 2010.

[60] X. Zhuge and A. Yarovoy, "Sparse multiple-input multiple-output arrays for high-resolution near-field ultra-wideband imaging," IET microwaves, antennas \& propagation, vol. 5, no. 13, pp. 1552-1562, 2011. 